\title{
The Trailing Edges of High-Speed Streams at 1 AU
}

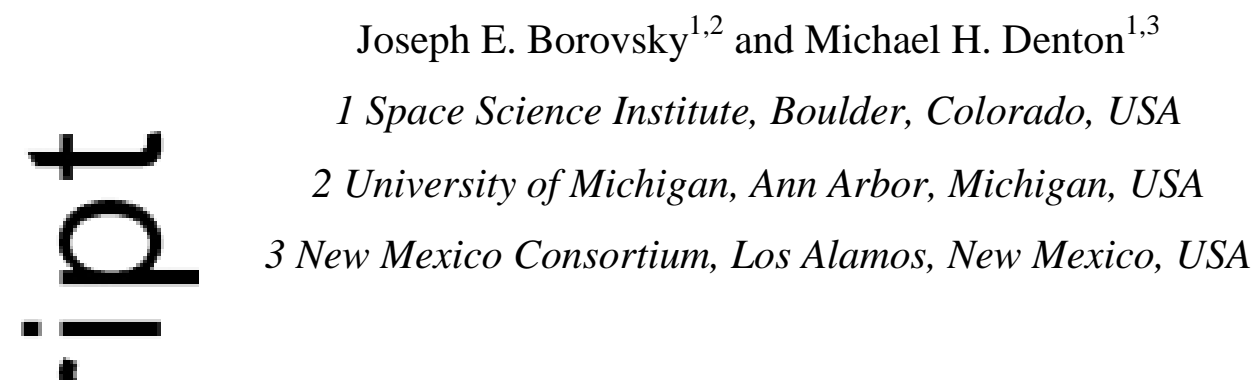

Abstrat The trailing-edge rarefactions of 54 high-speed streams at 1 AU are analyzed. The tempor duations of the trailing-edge rarefactions agree with ballistic calculations based on the observed speeds of the fast and slow wind bounding the rarefactions. A methodology is developed measure solar-wind compression and rarefaction using the orientations of solarwind curren sheets. One focus is to determine the signature that best describes the location of the trailing-edge stream interface between coronal-hole-origin plasma and streamer-belt-origin plasma based on the current-sheet orientations, on the magnetic-field strength, on the intensity of the ryon strahl, and on the intensity of the negative vorticity, an inflection point in the temporal profile of the solar-wind velocity is taken as the best indicator of the trailing-edge stream marface. Computer simulations support this choice. Using superposed-epoch analysis, the amoperties and turbulence properties of trailing-edge rarefactions are surveyed. Whereas the signatures of the coronal-hole/streamer-belt (slow-wind/fast-wind) boundary in the leading (CIR) stream interface are simultaneous, they are not simultaneous in the trailing edge, ith jon-charge-state signatures occurring on average 13.7 hours prior to the protonentropy signature. It is suggested that differences in the leading and trailing edges of coronal holes of the Sun might account for the differences in the leading and trailing edges of high-speed stream/at AU: the formation timescales, heating timescales, and charge-state-equilibration timescaresy closed flux loops in the corona might be involved.

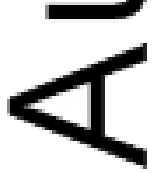

This is the author manuscript accepted for publication and has undergone full peer review but has not been through the copyediting, typesetting, pagination and proofreading process, which may lead to differences between this version and the Version of Record. Please cite this article as doi: 10.1002/2016JA022863 


\section{Introduction}

Because the rotating Sun has regions on its surface that produce wind of different speeds, along a radial line outward from the Sun will be regions of radially outflowing slow wind and regions of radially outflowing fast wind. On the leading (outward) edge of a region of high-speed wind (anigh-speed stream) the faster solar wind rams into slower solar wind and forms a region of plas na compression (a corotating interaction region -- CIR); on the trailing (inward) edge of a high-speed_stream the fast wind outruns the slow wind and forms a region of plasma rarefraction. The fasolar wind has its origin from coronal holes on the Sun; the slow solar wind has its origin fom treamer belts and streamer stalks on the Sun. In the CIRs on the leading edges of the high-speed streams there is a well-known sharp boundary making the transition from streamerbelt-or Alasma into coronal-hole-origin plasma known as the "CIR stream interface". By symmetry, the trailing-edge rarefaction there should be another stream interface making the transition from coronal-hole-origin plasma into streamer-belt-origin plasma: the "trailing edge stream nterface".

Luttrell, 1960); Pizzo, 1989, 1991; Crooker and Gosling, 1999; Jian et al., 2006; Tessein et al., 2011 , part because CIRs drive geomagnetic storms [Denton et al., 2006; Tsurutani et al., 2006.orovsky and Denton, 2006, 2013; Richardson et al., 2006; Lavraud et al., 2006; Solomon et al., 2012]. The trailing edges of high-speed streams are less studied [Carovillano and Siscoe, 1969; 19 and Marsch, 1995 Gosling and Pizzo, 1999; Pagel et al., 2004; Ko et al., 2006]: the trailing eages of high-speed streams drive the latter portions of high-speed-stream-driven geomagnetrc storms wherein geomagnetic activity is subsiding but the Earth's electron radiation belt is still being energized [Tsurutani et al., 1995; McPherron et al., 2009; Borovsky and Denton 2010a,b]. The CIR stream interface on the leading edges of high-speed streams has been well charized [e.g. Burlaga, 1974; Gosling et al., 1978; Crooker and Gosling, 1999; Forsyth and Marser, 1999; Intriligator et al., 2001; Simunac et al., 2009; Crooker et al., 2010; Borovsky and Part n, 2010c; Crooker and McPherron, 2012]. To identify the stream interface in leading- 
edge CIRs, various signatures have been utilized: the reversal of the east-west flow deflection of the solar wind [Gosling et al., 1978; McPherron and Weygand, 2006], the peak of the total (magnetic-field plus thermal) pressure [Jian et al., 2006], a sudden increase in the proton specific entropy [Siscoe and Intrilligator, 1993; Lazarus et al., 2003], a sudden change in the heavy-ion composition and in the FIP (first-ionization-potential) elemental abundance [WimmerSchwe gruer et al., 1997; Crooker and McPherron, 2012], or the peak in the plasma vorticity [Borovsluand Denton, 2010c]. Discussion of the stream interface in the trailing-edge rarefaction is quit limited [Burlaga et al., 1990; Geiss et al., 1995; Burton et al., 1999; Zurbuchen et al., 1999; Cahty, 2010; Simunac et al., 2010]. The most extensive study of the trailing edge stream interface 1s that of Burton et al. [1999], who examined 18 recurrences of a single persistent highspeed to at 13 - 36 degrees of latitude and 4.5 - 5 AU in distance from the Sun in the years 1992-1995. They recommend identifying the trailing-edge stream interfaces with a sharp drop in the proton specific entropy $S_{\mathrm{p}}$. They also found that drops in the proton specific entropy, jump increas $s$ in the $\mathrm{Mg} / \mathrm{O}$ elemental density ratio, jump increases in the oxygen charge-state ratio, and drone alpha-to-proton density ratio were all temporally coincident. Geiss et al. [1995] also forn abrupt signatures in the trailing edge that were simultaneous. Contrary to this, Zurbuthen et al. [1999] found the oxygen charge-state ratios to have gradual transitions across the thes jump increases; Simunac et al. [2010] also reported less-distinct changes in the trailing-edge rarefaction than in the leading-edge CIR, as did Pagel et al. [2004]. The prents study at $1 \mathrm{AU}$ will find that the various candidate signatures for identifying the stream (It) ace are typically not simultaneous and the present study will recommend a different signature to identify the location of the trailing-edge stream interface.

Note, the "trailing edge of a high-speed stream" should not be confused with the "CIR trailingedg. or "compression-region trailing edge" [cf. Richardson, 1985; Lucek and Balogh, 1998; Fburet al., 2012].

Intme present study 54 high-speed-stream trailing edges are examined at $1 \mathrm{AU}$ in the years 8-2008. The focus will be to (1) locate the stream interface in the trailing edge 
rarefaction, (2) to find a signature that can identify the stream interface, and (3) to survey the properties of trailing-edge rarefactions in general and in comparison with the properties of leading-edge CIRs. This study will use data from OMNI2, ACE, Wind, and Stereo A+B and the data interpretation will be aided by 3-D global-MHD simulations and by 1-D fluid simulations.

TData sets utilized in this study are OMNI2 [King and Papitashvili, 2005], ACE SWEP M McComas et al., 1998], ACE MAG [Smith et al., 1998], Wind SWE [Ogilvie et al., 1995. Kasner et al., 2006], Wind 3DP [Lin et al., 1995], Wind Mag [Lepping et al., 1995], Stereo ILASTIC [Galvin et al., 2008], and ACE SWICS [Gloeckler et al., 1998].

(this paper is organized as follows. In Section 2 expectations for the properties of trailing edges of high-speed streams as compared to the properties of corotating interaction regions on the lea $\mathrm{g}$ dges are explored. In Section 3 the event selection criteria and the collection of 54 high-speea-gream trailing edges are discussed. In Section 4 superposed-epoch analysis (with the help of computer simulations) is used to determine the best signature that identifies the stream interface in the trailing-edge rarefaction. In Section 5 superposed-epoch analysis is used to explore properties of high-speed-stream trailing edges in comparison with high-speed-stream leading ges. Section 6.1 discusses whether the initial velocity drop at the end of the high-speed streant the beginning of the trailing-edge rarefaction or whether the trailing-edge rarefaction compraces_earlier in time. Section 6.2 discusses the possibility that the differences between the leading-edge and trailing edge stream interfaces at $1 \mathrm{AU}$ have their origins in differences betwee the leading and trailing edges of coronal holes on the Sun. The findings of this study are summa zzee in Section 7 and a summary sketch of the chronology of signatures in the leading and trainm edges is presented. In Appendix A the methodology of measuring the amount of plasma compression or rarefaction in the solar wind with the orientations of current sheets in the plasmaris dincussed.

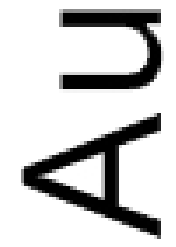




\section{The Trailing Edges of High-Speed Streams}

Figure 1 sketches the leading edge (left) and trailing edge (right) of a high-speed stream in the ecliptic plane as viewed from above the plane. The RTN coordinate system is noted in the bottom right of each sketch. In both cases the structures sketched move upward with time radiany outward from the Sun. In both sketches a green dashed line indicates the path of the Earth trough the structure as the structure moves radially outward from the Sun past the Earth, with_the Earth moving in the figure temporally from top to bottom along the line. In the left panel blue dashed line indicates the location of the CIR stream interface, which is a Parkerspiral-fiented interface between streamer-belt plasma (slow wind) and coronal-hole-origin plasma (fast wind). Shaded in orange is the corotating interaction region (CIR), a region of comprod plasma caused by the fast wind overtaking the slow wind. The corotating interaction region outy ard from the stream interface is compressed slow-wind plasma and the corotating interaction region inward of the stream interface is compressed coronal-hole-origin plasma (fast wind). The width of the CIR is related to the magnetosonic speeds in the fast and slow wind and the age the CIR. Nominally in the unperturbed slow and fast wind the velocity vectors (drawn in red) adially outward from the Sun. Within the CIR there is a pressure-driven velocity perturation perpendicular to the Parker spiral: this perturbation is indicated in the sketch by the gree arrows pointing outward from the stream interface. Within the CIR this velocity perturbation deflects the radially flowing fast wind into the - $\mathrm{T}$ direction and the radially flowing slow $n$ into the $+\mathrm{T}$ direction. This is the well-known westward-then-eastward temporal flow deflection a CIR passing over the Earth [e.g. Siscoe et al., 1969; Belcher and Davis, 1971]. The Clk-1s a large-scale pressure perturbation, not a large scale shear zone [Borovsky and Dentor 2010c]. There is a narrow, intense velocity shear (vorticity layer) at the stream interface, whichis a r glic from the Sun. In the left-hand panel of Figure 2 the creation of this vorticity layer at the slow-wind boundary at the Sun is sketched. The stream interface is depicted as the veried dashed black line, with slow wind (streamer-belt plasma and the sector-reversalregicn sma) to its left and fast wind (coronal-hole-origin plasma) to its right. The solar 
rotation is toward the left. Because of the change in the velocity across the stream interface, there is a vorticity $\underline{\omega}=\nabla \times \underline{v}$ across the stream interface. In the RTN coordinate system (noted on the sketch), the vorticity vector $\underline{\omega}$ is in the positive $\mathrm{N}$ direction.

In Figure 3 superposed-epoch averages of various quantities are plotted versus time for 27 leading-edge CIRs, with the zero epoch for the averaging being the time at which the streaminterfade voticity layer in each CIR reaches the ACE spacecraft. The $27 \mathrm{CIRs}$ are from the colletilized in Borovsky and Denton [2010c], where the event-selection criteria are discussindetail. In the top panel the solar wind speed $v_{r}$ as measured by ACE SWEPAM is plotted the slow wind prior to the stream interface followed by fast wind afterward is seen. In the second panel the transverse flow velocity $v_{t}$ is plotted: the positive $v_{t}$ then negative $v_{t}$ perturbaro across the CIR is seen. In the third panel the N component of the plasma vorticity $\underline{\omega}$ $=\nabla \times \underline{v}$ is pl tted: note the sharp localized peak at the stream interface. In the fourth panel the proton number density $\mathrm{n}_{\mathrm{p}}$, the proton temperature $\mathrm{T}_{\mathrm{p}}$, and the magnetic-field strength $\mathrm{B}_{\text {mag }}$ are plotted Cince $\mathrm{B}_{\mathrm{mag}}$ has about the same average value in coronal-hole-origin plasma (4.7 $\mathrm{nT}$ for the colegtyn of flattop high-speed streams at 1 AU [cf. Table 4 of Borovsky, 2016]) and in streamer-belt plasma (6.2 nT for the collection of pseudostreamer slow wind in Xu and Borovsky [2015]), the quantity $B_{\text {mag }}$ is a good indicator of the amount of compression or rarefaction that the suasma has undergone. Note the increase (compression) of $\mathrm{B}_{\mathrm{mag}}$ within the CIR in the fourth panel, peaking at the stream interface. In the fourth panel of Figure 3 the proton numbermity is also compressed within the CIR, but its value is not reliable as a measure of the am ount of compression because of the presence of "noncompressive density structures" [Gosling etal., 1981; Borrini et al., 1981] inside the CIR. These density structures are owed to the preance of sector-reversal-region plasma in the CIR [Xu and Borovsky, 2015], which occurs in hemereamer CIRs but not in pseudostreamer CIRs. Another measure of compression is to look at the anisotropy of the current-sheet orientations in the plasma, with the microscale magnetic structure of the solar-wind plasma flattening under compression and stretching under rarefatio . As developed in Appendix A, compression or rarefaction can be discerned by 
looking at the statistics of the directions of the current-sheet normal vectors in the plasma as the current sheets are advected past a measuring spacecraft. The normal direction $\underline{\mathrm{n}}$ of each current sheet is determined using the cross-product method [Burlaga and Ness, 1969; Knetter et al., 2004] on the magnetic-field vector change across the current sheet via $\underline{\mathrm{n}}=\underline{\mathrm{B}}_{1} \times \underline{\mathrm{B}}_{2} /\left|\underline{\mathrm{B}}_{1} \times \underline{\mathrm{B}}_{2}\right|$ where $\underline{B}_{1}$ and $\underline{B}_{2}$ are the magnetic-field vectors on the two sides of the current sheet. For Figure 3 only strong urrent sheets that had magnetic-field direction changes $\Delta \theta_{\mathrm{B}}>40^{\circ}$ across them were used to calcthe normal directions. If, in RTN coordinates, the normal has components $\left(\mathrm{n}_{\mathrm{r}}, \mathrm{n}_{\mathrm{t}}, \mathrm{n}_{\mathrm{n}}\right)$, then thentic angle of the normal can be defined as $\theta_{\text {out-of-eclpitic }}=\arctan \left(\left|\mathrm{n}_{\mathrm{n}}\right| /\left(\mathrm{n}_{\mathrm{r}}^{2}+\mathrm{n}_{\mathrm{t}}^{2}\right)^{1 / 2}\right)$. As dis usse in Appendix A, there is a reduction in the mean out-of-ecliptic angle $\theta_{\text {out-of-eclpitic }}$ of

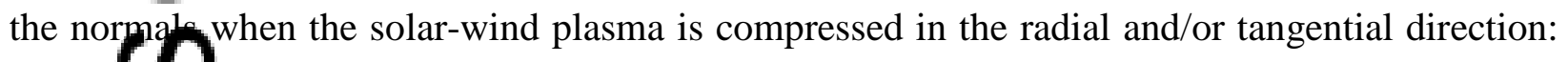
such a crecrease is seen around time $\mathrm{t}=0$ in the superposed average of the current-sheet normal angle in the fifth panel of Figure 3. In the sixth panel of Figure 3 the superposed average of the intensity of the 272-eV electron strahl as measured by ACE SWEPAM is plotted. In this panel the stra-intensity peaks just after the stream interface, as was reported by Crooker et al. [2010]. One pq ss pre explanation for this peak near the stream interface given by Crooker et al. [2010] is that the strahl is propagating from the Sun through a region of maximum compression where the solar-wint-magnetic-field lines are closest together and this closeness of the field lines preserves the of the electron strahl. (If that is the case, as a corollary we should expect the strength of the electron strahl to be a minimum at the trailing-edge stream interface where the rarefactonts the greatest, the field lines are furthest apart, and the strahl intensity is diluted the most.) n th bottom panel of Figure 3 the changes in the proton specific entropy $S_{p}=T_{p} / n_{p}^{2 / 3}$ and the heavy-ion charge-state-density ratios $\mathrm{C}^{6+} / \mathrm{C}^{4+}$ and $\mathrm{O}^{7+} / \mathrm{O}^{6+}$ are plotted: the transition in $\mathrm{S}_{\mathrm{p}}$ is an-aresse from streamer-belt (and sector-reversal-region) plasma into coronal-hole-origin plasnrand the charge-state ratios decrease from streamer-belt (and sector-reversal-region) plasmas inty coronal-hole-origin plasma.

In the right-hand panel of Figure 1 the expectations for the trailing edge of a high-speed strea sketched. Here fast wind leads slow wind and a Parker-spiral oriented rarefaction 
occurs as the fast wind advects away from the slow wind: the rarefaction is shaded in pink. Somewhere within the rarefaction is a Parker-spiral-oriented stream interface separating the coronal-hole-origin plasma from the streamer-belt-origin plasma: this stream interface is sketched as the red dashed line. As will be found in Section 4, identifying the stream interface in the tralmg-edge rarefaction is not straightforward. In the rarefaction there is a pressure-driven velocit penurbation perpendicular to and toward the Parker-spiral-oriented stream interface. This velocity perturbation is sketched as the green arrows in the right panel. Ahead (outward) of the stren interface, the velocity perturbation turns the radial flow of the fast coronal-hole-origin plasma into the - T direction and behind (inward) the stream interface it turns the radial flow of the slow streamer-belt plasma into the $+\mathrm{T}$ direction. For Earth moving down the green dotted line throug histern there will be an eastward-then-westward flow perturbation, opposite to the transverse now temporal pattern in a CIR.

The difference in the radial velocities of the fast wind and the slow wind will ballistically leave Parker-spiral-aligned gap between the fast plasma and the slow plasma. This gap will partially from pressure-driven expansion of plasma and field from both the fast and slow plasmas the gap. The expansion flows will be normal to the Parker-spiral direction and are seen the transverse flow deflections $v_{t}$ of the trailing edge. Simple time-of-flight arguments alon radial line can be used to estimate the temporal width of the gap as seen by a spacecraft at 1 AU. If the stream interface on the rotating Sun crosses the radial line connecting a spacecraft to the at time fast $r_{0} / v_{\text {fast }}$ and the front edge of the slow wind will ballistically arrive at the spacecraft at time $t_{\text {slow }}=r_{\mathrm{o}} / \mathrm{v}_{\text {slow }}$, where $\mathrm{r}_{\mathrm{o}}=1 \mathrm{AU}$ and where the $\mathrm{v}_{\text {fast }}$ and the $\mathrm{v}_{\text {slow }}$ are the speeds of the fast and sld $\mathrm{v}$ wind. The difference $\mathrm{t}_{\text {slow }}-\mathrm{t}_{\text {fast }}$ in these two arrival times is the temporal width $\Delta \mathrm{t}_{\text {gap }}$ of the garthetveen the slow wind and the fast wind:

$$
\Delta \Delta t_{\text {gap }}=r_{o}\left(v_{\text {slow }}{ }^{-1}-v_{\text {fast }}{ }^{-1}\right) \text {. }
$$

In the top panel of Figure $4 \Delta t_{\text {gap }}$ is plotted as a function of $v_{\text {slow }}$ for 5 different values of $v_{\text {fast }}$ (colas). The trailing-edge rarefaction between the fast and slow wind will be larger than this gap 
owing to the rarefaction waves that propagate into the fast and slow plasmas as those plasmas expand into the gap. Not accounting for the variation in the Parker-spiral orientation with distance from the Sun, and not accounting for the variation in the magnetosonic speed with distance from the Sun, the spatial widths of the rarefaction waves into the fast and slow plasmas at 1 AU are estimated as $\mathrm{L}_{\text {fast }}=\mathrm{C}_{\mathrm{ms} \text {-fast }} \mathrm{t}_{\text {fast }}=\mathrm{C}_{\mathrm{ms} \text {-fast }} \mathrm{r}_{\mathrm{o}} / \mathrm{v}_{\text {fast }}$ and $\mathrm{L}_{\text {slow }}=\mathrm{C}_{\mathrm{ms} \text {-slow }} \mathrm{t}_{\text {slow }}=\mathrm{C}_{\mathrm{ms} \text {-slow }} \mathrm{r}_{\mathrm{o}} / \mathrm{v}_{\text {slow }}$, where $\mathrm{C}_{\mathrm{ms}-\mathrm{f}}$ and $\mathrm{C}_{\mathrm{ms} \text {-slow }}$ are the magnetosonic speeds in the fast and slow plasmas. These spatial widths $\mathrm{L}_{\text {fast }}$ and $\mathrm{L}_{\text {slow }}$ at $1 \mathrm{AU}$ pass the spacecraft in the times

$$
1 \begin{aligned}
\Delta \mathrm{t}_{\text {fast }} & =\mathrm{L}_{\text {fast }} / \mathrm{v}_{\text {fast }}=\mathrm{C}_{\text {ms-fast }} \mathrm{r}_{\mathrm{o}} / \mathrm{v}_{\text {fast }}{ }^{2} \\
\Delta \mathrm{t}_{\text {slow }} & =\mathrm{L}_{\text {slow }} / \mathrm{v}_{\text {slow }}=\mathrm{C}_{\text {ms-slow }} \mathrm{r}_{\mathrm{o}} / \mathrm{v}_{\text {slow }}{ }^{2}
\end{aligned}
$$

For both the fast and the slow plasma at $1 \mathrm{AU}, \mathrm{C}_{\mathrm{ms} \text {-fast }} \sim \mathrm{C}_{\mathrm{ms} \text {-slow }} \sim 60 \mathrm{~km} / \mathrm{s}$. For $60 \mathrm{~km} / \mathrm{s}, \Delta \mathrm{t}_{\text {fast }}$ and $\Delta t_{\text {fast }}$ are plotted in the middle panel of Figure 4 as a function of $v_{\text {fast }}$ or $v_{\text {slow }}$. The total temporal wilth of a trailing edge $\Delta \mathrm{t}_{\text {trail }}$ is the sum of the three widths:

$$
\Delta \mathrm{t}_{\text {trail }}=\Delta \mathrm{t}_{\text {gap }}+\Delta \mathrm{t}_{\text {fast }}+\Delta \mathrm{t}_{\text {slow }} \quad \text {. }
$$

In the boum panel of Figure $4 \Delta t_{\text {trail }}$ is plotted as a function of $v_{\text {slow }}$ for 5 values of $v_{\text {fast }}$ (colors).

A 0 as the case for the CIR stream interface, there is a vorticity at the trailing-edge stream interface. In the right-hand panel of Figure 2 the origin of this vorticity at the Sun is depicted. the fast wind to the left of the stream interface (dashed black line) and the slow wind to the right, the vorticity vector $\underline{\omega}=\nabla \times \underline{\underline{v}}$ in the RTN coordinates system (noted on the sketch),is in the $-\mathrm{N}$ direction. At $1 \mathrm{AU}$, negative vorticity at the trailing-edge stream interface is anticipated.

[n F gure 5 a high-speed stream and its leading and trailing edges are plotted. In the top panel the wind flow $\mathrm{v}_{\mathrm{r}}$ is plotted as a function of time as measured by Wind SWE: The high-speed stream is indicated in red, as is the CIR (slow-wind to fast-wind transition) and the trailing edge (fast-wind to slow-wind transition). In the top panel two important features of the trailing edg are denoted. The first is the initial drop of the velocity: this is probably the leading edge of the arefaction propagating into the coronal-hole-origin plasma at the local magnetosonic speed. 
slope. The "v-bend" will be a candidate indicator of the location of the stream interface in the trailing edge. In the second panel of Figure 5 the proton specific entropy $S_{p}=T_{p} / n_{p}^{2 / 3}$ of the solar wind plasma is plotted logarithmically. A sharp drop of $S_{p}$ is seen within the trailing edge near the location of the velocity-bend. In the third panel of Figure 5 the heavy-ion charge-statedensity ratros $\mathrm{C}^{6+} / \mathrm{C}^{4+}, \mathrm{C}^{6+} / \mathrm{C}^{5+}$, and $\mathrm{O}^{7+} / \mathrm{O}^{6+}$ are plotted as measured by ACE SWICS: clear simult neou upward jumps of $\mathrm{C}^{6+} / \mathrm{C}^{4+}, \mathrm{C}^{6+} / \mathrm{C}^{5+}$, at $\mathrm{O}^{7+} / \mathrm{O}^{6+}$ are seen. These jumps in $\mathrm{C}^{6+} / \mathrm{C}^{4+}$, $\mathrm{C}^{6+} / \mathrm{C}^{5+}$ at $0^{7+} / \mathrm{O}^{6+}$ occur prior to the drop in $\mathrm{S}_{\mathrm{p}}$, while $\mathrm{S}_{\mathrm{p}}$ remains at the value in the high-speed stream In_oneral the downward drops of $\mathrm{S}_{\mathrm{p}}$ are more sudden than the upward jumps of $\mathrm{C}^{6+} / \mathrm{C}^{4+}$, $\mathrm{C}^{6+} / \mathrm{C}^{5}$ ang $\mathrm{O}^{7+} / \mathrm{O}^{6+}$. In the bottom panel of Figure 5 the elemental density ratios $\mathrm{C} / \mathrm{O}, \mathrm{Mg} / \mathrm{O}$, and $\mathrm{Fe} / \mathrm{O}$ are plotted as measured by ACE SWICS. An $\mathrm{Mg} / \mathrm{O}$ increase and a $\mathrm{Fe} / \mathrm{O}$ increase can be seer $($ De bottom panel.

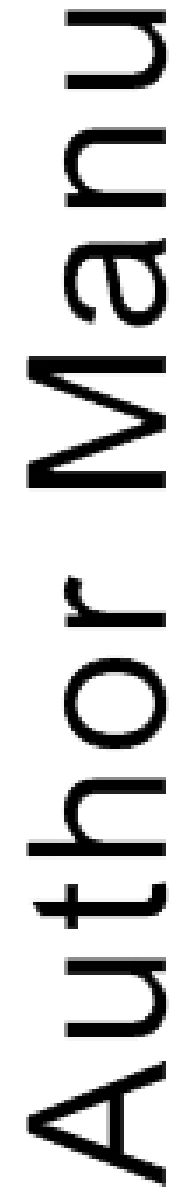




\section{Event selection}

A collection of 54 high-speed-stream trailing-edge rarefactions of high-speed streams is gathered by examining the solar wind in 1998-2008 using the OMNI2, ACE SWEPAM, and ACE SWICS data sets.

Four selection criteria are used to accept a trailing edge into the collection. The first criterin is that the trailing edge must be preceded by a robust high-speed stream with a duration of a davor_more. Flattop high-speed streams [Borovsky, 2016] are preferred. Second, a trailing edge isreiected if its temporal velocity profile is irregular. Profiles that are quasi-monotonic withou disgontinuities or multiple bend points are chosen. Third, a trailing edge is rejected if it contains clear signatures of ejecta. These signatures include depressed proton temperatures [Goslin elal., 1973; Elliott et al., 2005], long-duration out-of-ecliptic magnetic-field vectors [Leppingetgal., 2005; Borovsky, 2010], or bi-directional strahl [Gosling et al., 1987; Richardson and Cane, 2010]. The fourth criterion is that the velocity within the trailing edge should eventu 1ly reach low speeds ( $400 \mathrm{~km} / \mathrm{s}$ or less). I.e., the high-speed stream following the trailing cannot be too close in time to the high-speed stream preceding the trailing edge.

Thellection of 54 high-speed-stream trailing edges appears in Table 1. For each trailingedge, the start time (determined from the initial velocity drop at the end of the high-speed streane thermination time (determined as the point where the solar-wind velocity ceases to decline or starts to increase), the time of the velocity inflection or "velocity-bend", and the total time dusation of the trailing edge (from the initial velocity drop to the termination). Also noted in Table 1 se helmet-streamer versus pseudostreamer nature of the streamer-belt plasma in the trailing eure.

The total time duration of the trailing edges are listed in the final column of Table 1: the mean value nnd standard deviation of the durations are $89 \pm 34 \mathrm{hr}$. The velocity temporal decline in the edge is separated into two parts by the velocity-bend: the first part, which is a steep temporartacline in the solar-wind velocity prior to the velocity bend, has a duration of $31 \pm 18$ $\mathrm{hr}$ andrit second part, which is a shallow velocity temporal decline after the velocity-bend, has a 
duration of $57 \pm 25 \mathrm{hr}$. Typically, the second part of the velocity decline (velocity-bend to velocity termination) is about twice as long as the first part (initial velocity drop to velocity bend), but there is a wide range of variation. In Figure 6 the observed total time duration of each trailing edge in Table 1 is plotted as a function of the predicted width from expression (3) (using expressions (1) and (2)). As noted in the figure, the Pearson linear correlation coefficient betwee the observed width and the predicted width is $\mathrm{R}_{\text {corr }}=+0.79$. The blue dashed line in Figure 6 denotes the locations of the observed width when they exactly match the predicted width.

(The resulting collection of 54 high-speed-stream trailing edges contains 47 helmetstreamer trailing edges (being transitions from coronal-hole-origin plasma to helmet-streamerorigin $1 \mathrm{sp}$ a, having a single sector reversal in the slow wind) and contains 6 pseudostreamer trailing eages (being transitions from coronal-hole-origin plasma to pseudostreamer-origin plasma, without a sector reversal between the two high-speed streams); there is one trailing edge associa ed with a double sector reversal.

charach of the 54 high-speed-stream trailing edges the occurrence times of several
chatures are recorded: the initial velocity drop, the velocity-bend, the midpoint of the dropin $S_{p}$, the midpoint of the strong rise in $\mathrm{C}^{6+} / \mathrm{C}^{4+}$, the midpoint of the strong rise in $\mathrm{C}^{6+} / \mathrm{C}^{5+}$ the midpoint of the strong rise in $\mathrm{O}^{7+} / \mathrm{O}^{6+}$, and the termination time of the declining velocity profile. These characteristic features were located from visual inspection of temporal plots, ften with the aid of lines drawn upon the data plots. Some of these characteristic points will be asey to trigger superposed-epoch averages of solar wind measurements in the collection of high-speed-stream trailing edges. A goal is to discern which signature, if any, best describes the loc tion of the trailing-edge stream interface.

With less-stringent selection criteria, the authors also have a collection of 118 highspeed- trailing edges in the years 1998-2008. All of the findings that will be reported here for the nigh-speed-stream trailing edges also hold for the larger collection of 118 trailing edgecul st of the 118 trailing edges that were rejected to make the list of 54 higher-quality 
trailing edges were rejected because the pressure and velocity perturbation of the CIR following the trailing edge is encroaching into the trailing-edge rarefaction; hence the velocity of the trailing edge did not drop to low values and there was no reservoir of unperturbed slow wind at the end of the trailing-edge rarefaction.

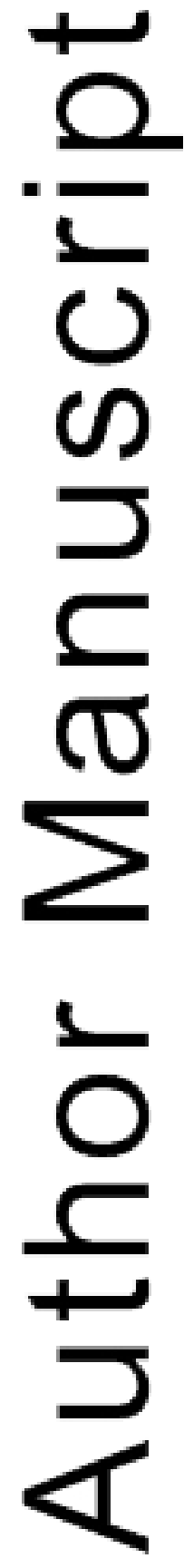




\section{Superposed-Epoch Analysis: Identifying the Trailing-Edge Stream Interface}

The stream interface is the boundary between coronal-hole-origin plasma and streamerbelt-origin plasma. Several methods have been used to identify the stream interface in CIRs (cf. Section 1), including the reversal of the east-west flow deflection of the solar wind, the peak of the totalpressure, a sudden increase in the proton specific entropy, the sudden change in the heavy- on c mposition and in the FIP elemental abundance, or the peak in the plasma vorticity. Using the zero crossing of the east-west flow reversal is problematic since there are east-west offsets the solar-wind flow that make it uncertain where to chose the single critical point in the broad everal; this idea is much improved by the vorticity method [Borovsky and Denton, 2010c] which analyzes the solar wind flows in a rotated local-Parker-spiral-oriented reference frame.

Trity signatures will be explored to identify the stream interface in the high-speed stream trailing edges: the sudden drop in the proton specific entropy $S_{p}$, the upward jump in the heavy ion ch ge state density ratios (with $\mathrm{C}^{6+} / \mathrm{C}^{4+}$ being the most-robust signature), and the velocitybend in themporal velocity profile. (Algebraic schemes to categorize the solar wind into its constituen lasma types have been developed based on both $\mathrm{O}^{7+} / \mathrm{O}^{6+}$ [Zhao et al., 2009] and on $\mathrm{S}_{\mathrm{p}}$ [Xud Borovsky, 2015].) The preferred signature to locate the stream interface would be a peak therticity, but in the individual high-speed-stream trailing edges isolated peaks in the vorticity are difficult to identify in the noise. Note that Burton et al. [1999] described factor-oftwo chinges in the FIP-effect density ratio $\mathrm{Mg} / \mathrm{O}$ as one of the signatures of the trailing-edge stream (tte face (a weak $\mathrm{Mg} / \mathrm{O}$ signature can be seen in Figure 5). Examining high-speed-stream trailing euges at $1 \mathrm{AU}$, the authors found that it is in general not possible to select an unamb guous signature in the $\mathrm{Mg} / \mathrm{O}$ 2-hr resolution SWICS measurements owing to large oscillations in the $\mathrm{Mg} / \mathrm{O}$ values. Other FIP-effect ratios such as $\mathrm{Si} / \mathrm{O}$ and $\mathrm{Fe} / \mathrm{O}$ were found to have similopehavior.

Tirtre first three panels of Figure 7 superposed-epoch averages of the solar wind velocity $\mathrm{v}_{\mathrm{r}}$ (te proton specific entropy $\mathrm{S}_{\mathrm{p}}$ (second), and the carbon charge-state-density ratio 
$\mathrm{C}^{6+} / \mathrm{C}^{4+}$ (third) are plotted: in each panel the four colors are superposed averages triggered on the initial v-drop (purple), the upward jump in $\mathrm{C}^{6+} / \mathrm{C}^{4+}$ (green), the drop in $\mathrm{S}_{\mathrm{p}}$ (blue), and the velocity-bend (red). The fourth panel plots the superposed average of the elemental density ratio $\mathrm{Mg} / \mathrm{O}$ with the same color scheme. In the top panel distinct features of the velocity profile are seen when the averaging is triggered on the initial v-drop (purple curve) and on the velocity-bend (red). Iotte in brown in the top panel is the superposed average of the solar-wind velocity with the ayeraging triggered on the velocity termination times that mark the end of the trailing edges. Again sharp velocity feature is seen at $t=0$ in this curve. In the second panel the plot of $S_{p}$ with the zer ep being the $\mathrm{Sp}$ drop (blue curve) shows a sharp drop signature at $\mathrm{t}=0$ since all of the individuar $S_{p}$ drops are lined up in the averaging. Likewise in the third panel of Figure 7 the plot of $\mathrm{C}^{6+}$ or the $\mathrm{C}^{6+} / \mathrm{C}^{4+}$-rise triggers (green curve) shows a somewhat-sharper feature at $\mathrm{t}=0$ : note that try superposed average of the $\mathrm{C}^{6+} / \mathrm{C}^{4+}$ rise (green curve third panel) is never as sharp as the superposed average of the $S_{p}$ drop (blue curve middle panel) because, in general, the individal $S_{p}$ drops are more sudden than the individual $\mathrm{C}^{6+} / \mathrm{C}^{4+}$ rises. In all panels of Figure 7 the curys the velocity-bend triggering and $S_{p}$-drop triggering are very similar: this is because the $\mathrm{S}_{\mathrm{p}}$ - $\mathrm{Cro}$ riggers and the velocity-bend triggers are close in time. Examining the time shifts of the fourcurves in the top panel the statistical time separation of the four trigger types are seen, with initial v-drop coming first, the $\mathrm{C}^{6+} / \mathrm{C}^{4+}$ jump coming next, and the velocity-bend and $\mathrm{S}_{\mathrm{p}}$ drop coming last. This trend is also seen in Figure 8 where the time intervals between the trigger times the individual trailing edges are binned for the 54 trailing edges: the $\mathrm{C}^{6+} / \mathrm{C}^{4+}$ trigger comes 0.7 hours after the initial v-drop on average (green curve), the $\mathrm{S}_{\mathrm{p}}$-drop trigger time comes 30.5 hours after the initial v-drop on average (purple curve), and the bend-v trigger comes $29.9 \mathrm{~h}$ urs after the initial v-drop on average (light-blue curve). The $\mathrm{C}^{6+} / \mathrm{C}^{4+}$ trigger is 14.0 hours ahead the bend-v trigger on average (red curve) and the $\mathrm{S}_{\mathrm{p}}$-drop is 0.5 hours ahead of the velocity on average (dark-blue curve). $\left(\mathrm{C}^{6+} / \mathrm{C}^{5+}\right.$ and $\mathrm{O}^{7+} / \mathrm{O}^{6+}$ triggers are at essentially the same as the $\mathrm{C}^{6+} / \mathrm{C}^{4+}$ triggers). The two signatures that are most-likely to be used to deternint the coronal-hole-plasma versus streamer-belt-plasma boundary are the $S_{p}$ drop and the 
$\mathrm{C}^{6+} / \mathrm{C}^{4+}$ jump (dashed black curve in Figure 8 ): on average the $\mathrm{C}^{6+} / \mathrm{C}^{4+}$ jump occurs 13.7 hours prior to the $S_{p}$ drop. Zurbuchen et al. [1999] also reported differences in the transition times in trailing edges, in particular between the transitions of charge states (coronal) and of elemental abundances (photospheric). In the bottom panel of Figure 7 the superposed average of $\mathrm{Mg} / \mathrm{O}$ is plottediror the four trigger signatures; here the superposed average of $\mathrm{Mg} / \mathrm{O}$ behaves somewhat like th supgrposed average of $\mathrm{C}^{6+} / \mathrm{C}^{4+}$ in the third panel, but noisier.

- In-Eigure 9 superposed-epoch averages of four critical diagnostics of the trailing-edge rarefaction are plotted as functions of time triggered on the three stream-interface candidate signaty es: Yhose candidate signatures are the upward jump in $\mathrm{C}^{6+} / \mathrm{C}^{4+}$, the drop in $\mathrm{S}_{\mathrm{p}}$, and the velocity-bend. In the top panel the superposed average of the magnetic-field strength $B_{m a g}$ in the 54 trai 2 dges is plotted. The decreased field strength within the trailing edge is caused by the rarefactrongf the plasma. In the top panel the $\mathrm{S}_{\mathrm{p}}$-drop triggering (blue curve) and the velocitybend triggering (red curve) yield lower values of $\mathrm{B}_{\mathrm{mag}}$ at the trigger point, implying that those signatu es are locating the center of the rarefaction better than the $\mathrm{C}^{6+} / \mathrm{C}^{4+}$-drop signature (green curve). mean freldrength in unperturbed coronal-hole plasma is $4.7 \mathrm{nT}$ for a collection of flattop highspeed treams at $1 \mathrm{AU}$ [cf. Table 4 of Borovsky, 2016] and $6.2 \mathrm{nT}$ for a collection of pseudstreamer streamer-belt plasma in $X u$ and Borovsky [2015]. Taking the mean of those two values, $5.5 \mathrm{nT}$ is an estimate of what the field strength would be when not rarefacted. In the top panel Eigure 9 the field strength in the center of the rarefaction is $3.1 \mathrm{nT}$, so the estimated rarefac onfactor is $5.5 / 3.1=1.8$. In the second panel of Figure 9 the superposed average of the out-of-ecmptic angle $\theta_{\text {out-of-eclipic }}=\operatorname{invtan}\left(\mid \mathrm{n}_{\mathrm{n}} /\left(\mathrm{n}_{\mathrm{r}}^{2}+\mathrm{n}_{\mathrm{t}}^{2}\right)^{1 / 2}\right)$ of current-sheet normals (see Appendix A) in te solar-wind plasma is plotted for the collection of 54 trailing edges. The elevated value of $\theta_{0}$ inside the trailing edges is an indicator of solar-wind magnetic-structure stretching in the pras ha owed to the rarefaction of the plasma. For a rarefaction by a factor of 1.8 (according to $\mathrm{B}_{\mathrm{mag}}$ ), the analysis of Appendix A predicts that the mean value of $\theta_{\text {out-of-eclipic }}$ shou 1 rease from $32.7^{\circ}$ to $44.3^{\circ}$ : this is approximately the effect seen in the second panel of 
Figure 9. In the second panel all three signatures (the upward jump in $\mathrm{C}^{6+} / \mathrm{C}^{4+}$, the drop in $\mathrm{S}_{\mathrm{p}}$, and the velocity-bend) do equally well (or equally poorly) at locating the maximum in the rarefaction as measured by the current-sheet anisotropy. In the third panel of Figure 9 the superposed-epoch average of the intensity of the 272-eV electron strahl is plotted for the three triggers. Crooker et al. [2010] suggested that the strahl intensity has a local maximum near the leading-edge stream interfa beause the strahl propagates away from the Sun there though a region that is maximally_ompressed and would have the solar-wind magnetic-field lines closest together to mainta the intensity of the strahl. If that is the case, we expect the strahl intensity to be a local minim ingar the trailing edge stream interface where the strahl propagates away from the Sun through a region that is maximally rarefacted. In the third panel the strahl intensity is locally minim( 1 ar the zero epoch for the $S_{\mathrm{p}}$-drop signature (blue curve) and for the velocity-bend signature (1) curve), more so than for the $\mathrm{C}^{6+} / \mathrm{C}^{4+}$-rise signature (green curve). In the bottom panel of Figure 9 the superposed average of the N-component vorticity $\omega$ of the plasma flow is plotted for the 54 trailing edges. The velocity-bend signature seems to be best at locating the maxim the negative vorticity, which is expected to occur at the stream interface.

In ing Figure 9 to judge which of the three candidate signatures (the upward jump in $\mathrm{C}^{6+} / \mathrm{C}$, the drop in $\mathrm{S}_{\mathrm{p}}$, or the velocity-bend) best denotes the location of the trailing-edge stream inter there is no clear choice. Further, there are high-speed-stream trailing edges that have non-monotonic velocity profiles with multiple bends, multiple $S_{p}$ drops, and/or multiple $C^{6+} / C^{4+}$ jumps. This is contrary to the impression of Burton et al. [1999], which was based on the examination of 18 recurrences of a single high-speed stream observed further out (4.5-5 AU) and at higher latitudes $\left(13-36^{\circ}\right)$ where trailing-edge rarefactions might behave differently.) Weigh ng the evidence in Figure 9, the velocity-bend signature does as well or better than the other the stream interface as diagnosed with $B_{\text {mag }}$ as a measure of

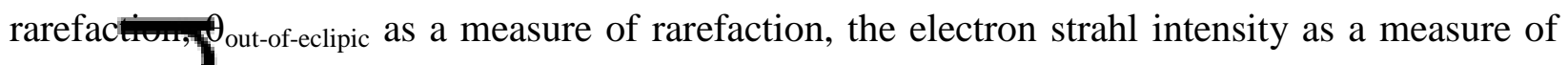
rarefaction, and $\omega$ as a measure of shear along the Parker spiral. The velocity-bend signature also 
has the advantage that it is robust and easily identified using plasma measurements. Often the velocity-bend can be located with a time accuracy of a minute or so.

Henceforth, the velocity-bend in the temporal velocity profile will be taken as the location of the stream interface in the trailing edge. This choice is validated by computer simulations.

ENDL 3-D MHD simulations [Odstrcil, 2003] of the solar wind in the heliosphere produced hy solar-wind emission from a rotating Sun display velocity breakpoints in the trailing edges fhioh-speed streams. In Figure 10 the solar-wind speed at Earth as predicted by ENLIL modelifg is plotted in black as a function of time for Carrington rotation 2057 in the year 2007. The first high-speed stream trailing edge in this plot is the example trailing edge of Figure 5. Noted 10 are distinct inflection points in the velocity profiles of three trailing edges. For ference, measurements of the solar-wind speed from Wind SWE are plotted in blue for the same time period.

To explore the origin of the velocity-bend in the trailing edges, one-dimensional fluid simulatiana-in spherical geometry of the solar wind advection and expansion out from the Sun are run.T fluid simulation code solves the equation of motion

$$
\partial \mathrm{v}_{\mathrm{r}} / \partial \mathrm{t}+\mathrm{v}_{\mathrm{r}}\left(\partial \mathrm{v}_{\mathrm{r}} / \partial \mathrm{r}\right)=-\left(1 / \mathrm{m}_{\mathrm{p}} \mathrm{n}\right) \partial \mathrm{P} / \partial \mathrm{r}
$$

on a we-dimensional grid in the radial direction to get updated values of $v_{r}(r)$ on the grid, then advects the plasma number density $n$, specific entropy $S_{p}$, and adiabatic index $\gamma$ outward with the local vaity $\mathrm{v}_{\mathrm{r}}(\mathrm{r})$ onto a new spatial grid. The advection scheme distributes the content of each old gri poryt forward in time onto the new grid with a conservative interpolation scheme. The simulation then uses an adiabatic law $\mathrm{T}=\mathrm{S}_{\mathrm{p}} \mathrm{n}^{\gamma-1}$ to compute the temperature $\mathrm{T}(\mathrm{r})$ on the grid from the vartues of $\mathrm{S}_{\mathrm{p}}(\mathrm{r}), \gamma(\mathrm{r})$, and $\mathrm{n}(\mathrm{r})$ on the grid, then calculates the pressure $\mathrm{P}(\mathrm{r})=\mathrm{nk}_{\mathrm{B}} \mathrm{T}$ on the grid. With the updated values of the pressure on the grid, a new timestep begins with the solution of expressio (4) for an updated $\mathrm{v}_{\mathrm{r}}(\mathrm{r})$ on the grid. To simulate the observed nonadiabatic addition of heat to solar wind as it cools expansively out from the Sun [cf. Borovsky and Gary, 2014], the efrowe adiabatic indices in Table 1 of Freeman and Lopez [1985] are used: in the 
simulations $\gamma=1.2$ is taken for slow solar wind and $\gamma=0.8$ is taken for fast solar wind. In Figure 11 a radial snapshot of a trailing edge evolving as it advects outward from the Sun is plotted as the black curves. In the top panel the solar wind velocity $\mathrm{v}_{\mathrm{r}}$ is plotted in black as a function of distance from the Sun, in the middle panel the normalized solar wind number density $\mathrm{nr}^{2}$ is plotted in black, and in the bottom panel the plasma thermal pressure $\mathrm{P}=\mathrm{nk}_{\mathrm{B}} \mathrm{T}$ is plotted in black. The in ut prameters at the Sun in this simulation are chosen such that for steady outflow from the Sun $=10 \mathrm{~cm}^{-3}$ at $1 \mathrm{AU}, \mathrm{T}_{\text {slow }}=5 \mathrm{eV}$ at $1 \mathrm{AU}, \mathrm{n}_{\text {fast }}=3 \mathrm{~cm}^{-3}$ at $1 \mathrm{AU}$, and $\mathrm{T}_{\text {fast }}=30 \mathrm{eV}$ at $1 \mathrm{AU}$. from $v$ st $=00 \mathrm{~km} / \mathrm{s}$ to $v_{\text {slow }}=350 \mathrm{~km} / \mathrm{s}$. In the black curve in the top panel note the inflection in the spatial profile of the flow velocity. To determine the origin of this velocity inflection, two other s. Alions are run. In one simulation (blue curves in Figure 11), the number density of the fast wind 15 reduced by a factor of $10^{4}$ to simulate the pressure expansion into "vacuum" of the slow wind outward off the front of the outward-advecting slow wind. In the other simulation (red curves Figure 11), the number density of the slow wind is reduced by a factor of $10^{4}$ to simulat thermal expansion into "vacuum" of the fast wind sunward off the back of the outwara-anecting fast wind. In the top panel of Figure 11 one can see that the blue and red curvestach follow the black curve until the vicinity of the black-curve inflection point is reached. This reement indicates that those portions of the black curve represent unimpeded pressure expansion of the slow wind and the fast wind into the trailing-edge rarefaction. In the bottom panel Eigure 11 a green arrow denotes the point at which the thermal pressures of the blue slow-y $x$ ansion into vacuum and the red fast-wind expansion into vacuum are close to equal. Fere, the slow-wind and fast-wind expansions will impede each other, forming an interfade between slow-wind plasma and fast-wind plasma. In the black curve of the top panel of Figure expansions impeding each other produces a velocity that locally has a reduced slope. plasma and the slow-wind plasma collide is a good point to choose for the stream interface. The green atr w from the bottom panel is reproduced in the top panel of Figure 11, showing the 
stream interface being very close to the location of the inflection point of the velocity spatial profile.

From the same black-curve simulation of Figure 11, the temporal profile of the solarwind speed at $1 \mathrm{AU}\left(215 \mathrm{R}_{\mathrm{s}}\right)$ is plotted in Figure 12 as the high-speed-stream trailing edge advects past the Earth. The initial drop and the velocity-bend in the temporal profile of the solarwind vocity are noted with green labels.

- The fluid simulations indicate that the inflection in the velocity profile is the point where the prosure expansions into the gap of the slow and fast plasmas meet: the slow plasma expand (ng Trward (away from the Sun) and the fast plasma expanding backward (toward the Sun).

Foure 13 the fraction of time that the $X u$ and Borovsky [2015] algebraic categorization scheme (will ch is based on the proton specific entropy $S_{p}$, the proton Alfven speed $v_{A p}$, the proton temperature $\mathrm{T}_{\mathrm{p}}$, and the solar-wind speed $\mathrm{v}_{\mathrm{sw}}$ ) for the solar-wind plasma yields coronalhole-or gin plasma (red curves), streamer-belt-origin plasma (blue curves), sector-reversal-region plasma curves), and ejecta (purple curves) is plotted for the collection of 54 high-speedstream rang edges of Table 1. In the top panel the time is organized about the initial velocity drops the 54 trailing edges. Here it is seen that the initial velocity drop (vertical dashed line) corre and the probability of streamer-belt-origin plasma is changing from $0 \%$. In the second panel of Figure 3 the data is organized according to the time of the velocity-bend in each trailing edge. In the ateg rization scheme the velocity-bend signature is occurring about where the occurrence probability of coronal-hole-origin plasma approaches $0 \%$ and where the combined probability of stream(r-belt-origin and sector-reversal-region plasma approaches $100 \%$. In the bottom panel of Figure 13 time is organized according to the velocity terminations of the 54 trailing edges. The velocitymation signature (vertical dashed line) is occurring at a location where the categorizatron scheme says the probability of coronal-hole-origin plasma approaches $0 \%$ and the probabirty of seeing streamer-belt plasma is becoming a local minimum, and the probability of 
seeing sector-reversal-region plasma is becoming maximum. Unfortunately, there are not enough pseudostreamer trailing edges in the Table 1 collection to separately perform superposed epoch analysis for helmet-streamer versus pseudostreamers; pseudostreamers have no sector-reversalregion plasma in them.

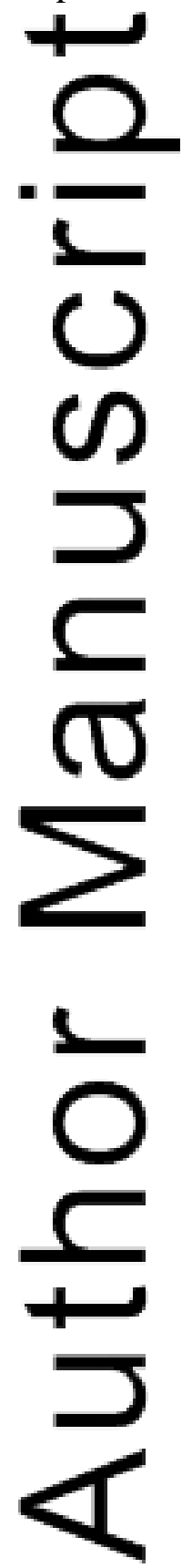




\section{The Properties of the Trailing Edges of High-Speed Streams}

In this section the properties of the trailing edges will be studied using superposed-epoch analysis triggered on the velocity-bend signatures (taken as the trailing edge stream interface) and on the termination times of the trailing edges. In Section 6.1 the analysis will continue triggerta on the initial velocity drop (taken as the onset of the trailing edge). This superposedaverag stuy could have been performed by temporally stretching each time series for the individualtrailing-edge rarefactions to line up simultaneously all of the velocity-bend signatures, all of he-termination times, and all of the initial velocity drops. In some sense this would simplif the display of data, needing only one figure instead of three figures. But the choice was made to look at the superposed-epoch averaging individually for each type of trailing-edge critical onture.

Trgore 14 examines the basic properties of high-speed-stream trailing edges in contrast to the examination of CIRs in Figure 3. The top panel of Figure 14 plots the superposed average of the sol rwind speed as a function of time, with the zero epoch of the 54 trailing edges being the velocity in the temporal velocity profile of each trailing edge: the fast wind before and the slower after is seen, with the bend in the velocity profile readily apparent. In the second paner thansverse velocity $\mathrm{v}_{\mathrm{t}}$ has the pattern $-\mathrm{v}_{\mathrm{t}}$ first then $+\mathrm{v}_{\mathrm{t}}$, opposite to the temporal pattern of $v_{\text {TIR }}$ (see figure 1 and the second panel of Figure 3). In the third panel the plasma vorticity is negative in the trailing edge (cf. Figure 2), with a negative peak just prior to the velocit-bend. In the fourth panel of Figure 14, no sharp boundary in the plasma parameters of the sol mind are seen, however there is an upward inflection of the superposed average of the proton number density at the time of the velocity-bend: this may be an indication of a transition from cronal-hole plasma to streamer-belt plasma with streamer-belt plasma having on average higherdensities than coronal-hole plasma. In the fifth panel the superposed average of the outof-eclipingle of the current-sheet normals in the solar-wind plasma indicates a stretching (rarefactivir) of the mesoscale magnetic-field structure of the solar-wind plasma in the trailing edge a peak just prior to the velocity-bend at $\mathrm{t}=0$. The superposed average of the intensity 
of the 272-eV electron strahl is plotted in the sixth panel of Figure 14. In this panel the strahl intensity is a minimum at the trailing-edge stream interface; this is expected in analogy to the Crooker et al. [2010] suggestion that the strahl is maximum where the compression is maximum; here the strahl is minimum where the rarefaction is maximum. In the bottom panel of Figure 14 the downward transition of the proton specific entropy $S_{p}$ and the upward transitions of the heavy-on carge-state-density ratios are seen, with the $S_{p}$ transition occurring after the heavyion trapsition in the superposed averages.

In Figure 15 some of the Alfvenic properties of the solar-wind plasma are explored via superp sed-poch averaging triggered on the velocity-bend of each trailing edge in the collection of 54. In the top panel the superposed average of the signed Alfvenicity [cf. Borovsky and Denton $(010 \mathrm{cc}$

$$
\longrightarrow \quad A=s \delta \underline{v} \bullet \delta \underline{B} /|\delta \underline{v}||\delta \underline{B}|
$$

is plotted, where the $\operatorname{sign} s(t)=+1$ if the IMF is away from the Sun and $s(t)=-1$ if the IMF is toward the Sun. Positive A represents outward-sense Alfvenicity of the solar-wind $\underline{\mathrm{v}}$ and $\underline{\mathrm{B}}$ fluctua 10 is and negative A represents inward-sense Alfvenicity. In the top panel A is calculated on a 128-S timescale with ACE SWEPAM and ACE MAG. The superposed average of A is positive the plot, with a decay in the Alfvenicity across the trailing edge. At the velocind $(\mathrm{t}=0)$ there is a non-monotonic inflection in the superposed average of $A$ : the statistical significance of this inflection is difficult to gauge, as is the possible physical origin of the inflection. In the second panel of Figure 15 the superposed average of the amplitudes of the outwar (reg) and inward (blue) Elsasser fluctuations are plotted. The Elsasser amplitiudes $\delta \underline{v} \pm$ $\delta \underline{B} /\left(4 \pi \mathrm{pm}_{1}^{1 / 2}\right.$ [e.g. Tu and Marsch, 1995; Bruno and Bavassano, 1991] are calculated using ACE velocing metic-field, and density measurements with 64-sec time resolution and the amplitudes are plotted for the frequency range $\mathrm{f} \geq 1 \times 10^{-3} \mathrm{~Hz}$. The amplitude of outward Alfvenic fluctuations decays strongly prior to the v-drop (stream interface) at $t=0$ and ceases to decay thereafter The amplitude of the inward Elassser fluctuations decays slightly in the trailing edge prior to $\mathrm{e}$-drop point at $\mathrm{t}=0$ and then does not decay thereafter. In the third panel of Figure 15 
the superposed average of the outward to inward Elsasser-variable amplitudes is plotted: the strong outward imbalance decays in the trailing edge prior to the v-drop point (stream interface) and does not decay appreciably afterward. Note the non-monotonic inflection in the superposed average of the ratio of Elsasser amplitudes in the third panel looking very much like the inflection in the signed Alfvenicity in the top panel. In the bottom panel of Figure 15 the superp sed average of the normalized alpha-particle beam speed in the solar wind (measured with„Wind 2DP) $\left|\underline{\mathrm{v}}_{\text {alpha }}-\underline{\mathrm{v}}_{\text {proton }}\right| \mathrm{v}_{\mathrm{Ap}}$ (normalized to the proton Alfven speed $\mathrm{v}_{\mathrm{Ap}}=\mathrm{B} /\left(4 \pi \mathrm{m}_{\mathrm{p}} \mathrm{n}_{\mathrm{p}}\right)^{1 / 2}$ ) is plott_ Is is well known that the normalized beam speed is larger in the outward Alfvenic region of the fast solar wind [e.g. Marsch et al., 1982] and smaller in the less-Alfvenic slower wind [Hirschberg et al., 1974; Asbridge et al., 1976; Marsch et al., 1982]. In the bottom panel of Figure the decay of the normalized beam speed is seen in the trailing edge before the stream interface at $=0$ and the decay is significantly less thereafter.

In the five panels of Figure 16 the plasma properties of the latter portions of trailing-edge rarefacions are investigated using superposed-epoch analysis triggered on the velocity terminat of the trailing edges in Table 1. In the top panel the superposed average of the solarwind spee 1 s plotted: the long temporal decline of the velocity is clearly seen, with the velocity termination at $\mathrm{t}=0$, which is the chosen trigger time. In the superposed average an increase in the solar somed commencing at $\mathrm{t}=0$ appears: not all of the individual events have such increases, but none have decreases so the superposed average shows a net increase. The second panel of Figure 16 lots the superposed average of the transverse velocity $v_{t}$ of the solar wind. The tempor pagtern negative $v_{t}$ then positive $v_{t}$ of the trailing edge is seen in the days prior to the termination of the trailing edge at $\mathrm{t}=0$. The second panel indicates that the positive $\mathrm{v}_{\mathrm{t}}$ signature of the traing edge peaks near the termination of the trailing edge: such a peaking of the transverse velocityat the end of the trailing-edge rarefaction is not seen in computer simulations (cf. Fig. 12 of Pizz-91]). To verify the reality of this systematic positive-vt flow in the very slow solar wind, superposed-epoch averages of $v_{t}$ are plotted in Figure 17 using data separately from the Wincory Faraday cup (red curve) and the ACE SWEPAM electrostatic analyzer (blue curve). 
The two instruments agree, indicating the reality of this systematic signal. One contributor to this positive transverse flow velocity $\mathrm{v}_{\mathrm{t}}$ could be the onset of CIR events at the termination of some of the trailing-edge rarefactions. CIR onsets in the statistics also explains some of the increase in the solar-wind velocity after $\mathrm{t}=0$ in the top panel. When events that have velocity increases near to the verocity terminator are eliminated, the superposed average of $v_{t}$ still shows this positive maximg the time of velocity termination. The reduction of the superposed average of the out-ef-eclintic angle $\theta_{\text {out-of-ecliptic }}$ of the current-sheet normal vectors in the fourth panel of Figure 16 also indicates the presence of CIR compressions after time $\mathrm{t}=0$, although this reduction may be in fart caused by the presence of aligned current sheets near the heliospheric sector boundaries [e.g. Nakagawa et al., 1989; Nakagawa, 1993; Crooker et al., 1993, 1996, 2001]. The increas ( 2 bove $32^{\circ}$ ) value of $\left\langle\theta_{\text {out-of-ecliptic }}\right\rangle$ before $t=0$ indicates the broad rarefaction of the trailing edg. The third panel of Figure 16 plots the superposed average of some plasma properties: the magnetic-field strength (blue curve) indicates the broad rarefaction before $\mathrm{t}=0$. The reduced intensity of the electron strahl in the fifth panel also supports this broad-rarefaction picture ${ }^{0}$ in the fourth panel the rise in the plasma number density (red curve) prior to $t=0$; this risemay in part be caused by the increased likelihood that sector-reversal-region plasma is being emsuntered, with its enhanced density clumps. In the bottom panel of Figure 16 the supe anderages of the ion-charge-state density ratios $\mathrm{C}^{6+} / \mathrm{C}^{4+}$ and $\mathrm{O}^{7+} / \mathrm{O}^{6+}$ are plotted logarithmically along with the proton specific entropy $S_{\mathrm{p}}$ : note that $\mathrm{C}^{6+} / \mathrm{C}^{4+}$ and $\mathrm{O}^{7+} / \mathrm{O}^{6+}$ decline with timight up to the end of the trailing edge at $t=0$, and that $S_{p}$ increases with time right up to the end the trailing edge. This supports the earlier reports [e.g. Pagel et al., 2004; Zurbuchen et al., 1999; Simunac et al., 2010] of gradual transitions rather than abrupt transitions of the lasma properties.

In Eure 18 the properties of the fluctuations in the latter portions of high-speed-stream trailing touges are examined: again, superposed-epoch averaging triggered on the velocity terminations of the trailing edges is used. The top panel plots the superposed average of the signe venicity (expression (5)); the fast-wind/slow-wind transition of the signed Alfvenicity 
from high values to low values across the long trailing edge prior to $t=0$ is seen. In the second panel of Figure 18 the superposed averages of the outward-sense (red) and inward-sense (blue) Alfvenic fluctuations of $\underline{\mathrm{v}}$ and $\underline{\mathrm{B}}$ are plotted. The decay of the amplitude of outward fluctuations from faster solar wind to slower solar wind is seen across the trailing edge in the days prior to $\mathrm{t}=0$. The superposed average of the ratio of the Elsasser amplitudes in the third panel agrees with this, w th thecay of the outward imbalance being caused by the decreasing amplitudes of the outward-sense Alfvenic fluctuations. The bottom panel of Figure 18 displays the decrease in the normaned speed of the alpha-particle beam in the solar-wind plasma: the temporal profile of the beam sfeearesembles the temporal profile of the outward imbalance in Elsasser amplitudes.

The early portions of the trailing edges are examined separately in Section 6.1, where the onset $\mathrm{a}$ ing edge is the focus of the discussion.

Frgure 19 examines the robustness and reproducibility of the properties of high-speedstream tralling edges. In the top panel of Figure 19 a high-speed-stream trailing edge (Trailing Edge 45 of Table 1, see also Figure 5) is viewed by three spacecraft at 1 AU with relatively close semations. In the top panel Stereo A and Stereo B are separated by $10^{\circ}$ of longitude (which 15 days of solar rotation), with ACE and Wind (OMNI) in between the two. In the top pane the velocity profile of the trailing is reproducible on the three spacecraft, with smallscale atations of $v_{r}$ not reproduced from spacecraft to spacecraft. Note that the variation in $v_{r}$ between the three spacecraft is greater in the high-speed stream than it is in the trailing-edge rarefaction_see also Borovsky [2016] for an examination of spatial structure in coronal-holeorigin oray wind plasma). In the second panel of Figure 19 Trailing Edge \#47 of Table 1 is examinea. nere Stereo A and Stereo B are separated by $40.4^{\circ}$ of longitude and by $5.4^{\circ}$ of latitude, with $\mathrm{OMNI}$ between the two. $40.4^{\circ}$ of longitude is 3.1 days of solar rotation. In this panel the velocity nrofile of the trailing-edge rarefaction is not very reproducible, and features like the initial aity drop, the velocity-bend, and the velocity termination have quite different appearames in the three spacecraft cuts through the trailing edge. Note in the second panel that the stret re of the high-speed stream preceding the trailing edge is very irreproducible between 
the three spacecraft views. In the third panel of Figure 19 Trailing Edge \#53 of Table 1 is examined: here Stereo A and Stereo B are separated by $61.5^{\circ}$ of longitude (4.6 days of solar rotation) and by $5.8^{\circ}$ of latitude, with OMNI between the two. In this panel the velocity profile of the trailing-edge rarefaction is not very reproducible, i.e. the initial velocity drop looks different and therverocity-bend is sometimes difficult to identify. Again, in the third panel the structure of the high-sped stream shows great variations from cut to cut by the three spacecraft. Figure 19 indicates that it is a matter of luck whether a particular trailing-edge rarefaction meets the criteria of Section 3 to make it into the collection or whether it is deemed to be non-monotonic or irregul A. Ayditionally, measurements of quantities like the temporal width of the trailing edge will have some variability.
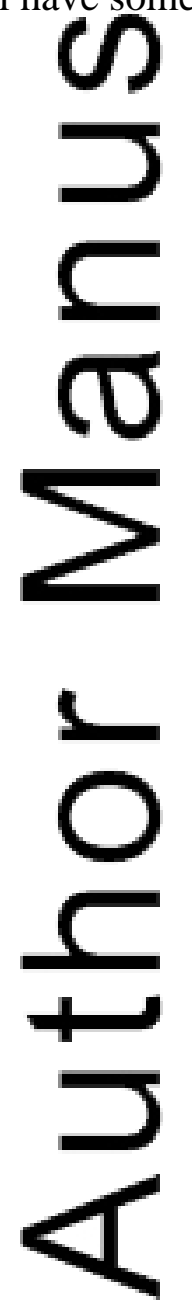


\section{Discussion}

This section contains two discussions. The first (Section 6.1) is a continuation of the examination of the properties of high-speed-stream trailing edges from Section 5, organized under the question of whether or not the initial velocity drop is the initiation of the trailing-edge rarefaction. The second discussion (Section 6.2) examines the leading and trailing edges of corona holfs on the Sun for clues about the origins of differences that are seen between the leading-edoe stream interface and the trailing-edge stream interface seen in the solar wind at 1 AU.

\subsection{Is he Mpitial Velocity Drop the Onset of the Trailing-Edge Rarefaction?}

The initial velocity drop, assumed to be at the termination of the high-speed stream, has been $\mathrm{t}$ o be the front of the rarefaction wave propagating antisunward into unperturbed coronafrory-origin plasma, but that might be a misidentification.

Borovsky [2016] collected 66 intervals of solar-wind data at 1 AU that provided the best candid tes for intervals of "unperturbed coronal-hole-origin plasma": the intervals selected were "flattop" high-speed streams, wherein the solar-wind velocity reaches a high level and then persists at high level (with high-frequency noise) for a day or more. The temporal plot of $v_{r}$ showedflat tops" on the high-speed streams. In making that collection it was clear that the early portion of the flattops had to be eliminated from the data collection as being perturbed (compressed or rarefacted) because of elevated magnetic-field strengths indicating potential comprosion. Regardless of the seeming evidence of compression, the velocity was often constan III hese eliminated early portions. When the solar-wind parameters were studied with superposea-epoch averaging triggered on the onsets of the collected flattop regions, solar-wind parame ers were in general constant in the early portions of the flattops (after the elimination of the marnetis-field-perturbed portions). However, when the superposed averages were triggered on the flattop regions, the latter portions of the flattops showed systematically decreasimg values of the proton number density and the magnetic-field strength, even though the velocitayed constant. No systematic variation of the proton specific entropy or heavy-ion 
charge-state ratios was seen in the latter portions of the flattops. Except for the lack of a velocity variation, these observed trends could be indications of either (1) a rarefaction wave moving plasma into the trailing-edge gap or (2) super-radial expansion effects near the Sun at the edge of a coronal hole.

Figure 20 contains a layout of superposed averages triggered on the initial velocity drops (vertic das hed line) of the 54 trailing edges of the current study. The third panel of Figure 20 corroberates the temporal decline of $\mathrm{n}_{\mathrm{p}}$ and $\mathrm{B}_{\mathrm{mag}}$ in the latter portions of the high-speed streams reportedin_Borovsky [2016]. The second panel of Figure 20 indicates a strong positive transverse velocit $\mathrm{V}_{\mathrm{t}}$ and prior to the initial velocity drop, consistent with plasma pressure expansion into the trailing-edge rarefaction at these locations. In the fourth panel of Figure 20 the mean value of the curet normal orientations $\theta_{\text {out-of-ecliptic }}$ is above $32.7^{\circ}$ in the region before and at the initial velogty drop: according to the analysis of Appendix A (see figure therein) this would indicate that the solar-wind plasma in these regions has undergone some rarefaction. Edge-ofthe-conal-hole effects at the Sun might lead to magnetic structure that is unidirectionally stretcherurth panel), but it would not lead to transverse flows at 1 AU (second panel). The decay or the superposed average of the electron-strahl intensity before the initial velocity drop in the six panel of Figure 20 also seems to supply evidence for a rarefaction prior to the initial velo drop, however Borovsky [2016] found that the intensity of the electron strahl systematically decays all the way across flattop high-speed streams (in individual cases and in the suptrnosed averages). This total decay, of which the decay in the sixth panel is part of, has not bee $e_{\text {plained. }}$

Figure 21 examines some of the fluctuation properties of the trailing edge with superpsed-epoch averaging triggered on the initial velocity drop. In the top panel the supernsed nverage of the signed Alfvencity (expression (5)) is plotted: the Alfenicity of the velocit magnetic-field fluctuations steadily declines from before the initial velocity drop until a Iew days afterward. In the second panel the amplitude of the outward Alfvenic flucturto is decreases significantly from before the initial drop until a day or so afterward, while 
the superposed average of the inward amplitude declines only slightly. The outward imbalance of the fluctuations (third panel) has a decrease that begins prior to the initial velocity drop but the majority of the decrease is afterward. In the bottom panel of Figure 21 the superposed average of the normalized alpha-beam speed in the solar-wind plasma is plotted: the majority of the decrease in the beam speed comes after the initial velocity drop. Figure 21 finds that the changes in the ropeties of the fluctuations in the solar-wind plasma begin, for the most part, prior to the initial velocity drop. This is a further indication that the rarefaction wave front may be prior to the inith velocity drop.

(f) thy rarefaction wave has propagated antisunward past the initial-velocity-drop point, then (1) the beginning of the trailing-edge rarefaction is at an as yet unidentified point before the initial 6 ty drop (by about a day) and (2) the initial velocity drop is a different feature in the trailingeagy with an as yet unidentified origin.

\subsection{The Leading-Edge and Trailing-Edge Stream Interfaces in the Solar Wind and the Leadingand Trailing Edges of Coronal Holes}

Theading-edge (CIR) stream interface at 1 AU has nearly simultaneous signatures (to withirthe 1-hr time resolution of the heavy-ion measurements): a sudden increase of the proton specifentropy, a decrease of the heavy-ion charge-state level, and localized positive vorticity. The trailing-edge stream interface has much less simultaneity of its signatures, as summarized in figure in the trailing edge of the high-speed stream the different boundaries are typically separat amy time by about 12 hours.

Owing to the compression of the solar-wind plasma in the leading edge and the rarefacion of the solar-wind plasma in the trailing edge, one would expect that the sequence of signatures would be stretched out in time in the trailing edge in comparison with the leading edge. Howevis compression-rarefaction time stretching is not enough to explain the timing of signatures in the trailing edge. Looking at the magnetic-field strength as a measure of compress on and rarefaction (as in Section 4), the superposed average of the magnetic field 
strength in the CIR at the stream interface (fourth panel of Figure 3) yields $\mathrm{B}_{\mathrm{mag}}=10.3 \mathrm{nT}$ at the compressed leading-edge stream interface and the superposed average of the magnetic field strength in the trailing edge (fourth panel of Figure 14) yields $B_{\operatorname{mag}}=3.1 \mathrm{nT}$ at the rarefacted trailing-edge stream interface. Taking the ratio of these two field strengths gives a measure of the ratio or the compression to the rarefaction, which is a factor of $10.3 / 3.1=3.25$. Hence time should be s retched by about a factor of about 3.25 in the trailing edge relative to the leading edge. This factor of 3.25 is not sufficient to account for the large differences in the times of the variouscionatures in the trailing edge (Figure 8). There is also in general an absence of strong transition signatures of charge-state-density ratios and of the proton specific entropy in the trailing edges. The transitions in $\mathrm{C}^{6+} / \mathrm{C}^{4+}, \mathrm{O}^{7+} / \mathrm{O}^{6+}$, and $\mathrm{S}_{\mathrm{p}}$ from coronal-hole-origin values to stream 1 torigin values are dominated by long gradual transitions (cf. Zurbuchen et al. [1999], rasfel et al. [2004], or Simunac et al. [2010]) with jumps that are not particularly large nor particularly sudden (cf. third panel of Figure 5 or panels 2 and 3 of Figure 7).

One explanation of the differences in the timing of signatures in the leading versus trailing atros at $1 \mathrm{AU}$ could be that solar-wind production differs at the leading and the trailing edges ononal holes. In figure 22 a coronal hole with its leading and trailing edges is sketched. The equatorial extensions of coronal holes tend to exhibit rigid rotation [e.g. Timothy et al., 1975 dams, 1976; Hiremath and Hedge, 2013] whereas the photosphere rotates differentially: in this mismatch coronal holes can lead the rotation of the photosphere. This produces a major differe be between the leading and trailing edges of a coronal hole: in the reference frame of the corona (IIO) at the westward leading edge the photosphere enters the floor of the coronal hole from the streamer belt and at the eastward trailing edge the photospheric floor of the coronal hole exits the coronal hole moves into the streamer belt (cf. Fig. 1 of Kahler [2010]). As noted in Figure 22, the "age" of the coronal hole floor (with age being how long it has had open flux) increas o fring from the westward leading edge to the eastward trailing edge [cf. Borovsky, 2016]).Imerchange reconnection is observed to occur on the edges of coronal holes [Baker et al., 2007. Sut ramanian et al., 2010; Krista et al., 2011]; owing to this inflow-outflow difference the 
nature of the reconnection of open flux with closed loops may differ on these two edges [cf. Fisk et al., 1999a,b; Wang and Sheeley, 2004], particularly concerning the properties of the plasma on the closed flux loops that feed plasma into the open flux tubes [Kahler et al., 2010]. Indeed, differences in westward-edge versus eastward-edge reconnection signatures have been reported [Yangetat., 2011], with more reconnection jetting observed on the leading (westward) edge than on the railing (eastward) edge. The magnitudes of the differences between the trailing-edge and the leadino-edge stream interfaces may depend on whether the high-speed-stream source is a rigidly otating extension of a polar coronal hole versus a differentially rotating isolated coronal hole [Z rken 1977; Nash et al., 1988 Navarro-Peralta and Sanchez-Ibarra, 1994].

As noted in Figure 22, the streamer-belt region adjacent to the westward leading edge is old, in 1 ense that its flux been closed for a long time; high, well-developed arches should populate Iny region. On the contrary, the streamer-belt region adjacent to the eastward trailing edge is young in the sense that the photosphere there recently was an open-flux region; here the arches may not be so well-developed, lacking high hot closed arches. As the open flux reconneatawith the uppermost arches, high hot arches will feed plasma into the open flux at the leadinged whereas lower and cooler arches will feed plasma into the open flux at the trailing edge. 10 ith height corresponding to the electron temperature and hence to the charge-state ratio eldman et al., 1999; Gloeckler et al., 2003], a gradual variation in the loop sizes on the Sun may map into a gradual transition of heavy-ion charge-state ratios in the solar wind.

To elaborate further, as sketched in Figure 22 there might be a wake-like diffuse bounda yoy the eastward trailing edge of the coronal hole making a slow temporal transition on the Sun rrom open-flux coronal-hole photosphere to closed-flux streamer-belt photosphere. As the ph tosphere goes into a closed-flux configurations on the trailing edge of a coronal hole, there will by three important timescales: (1) the timescale for the formation of high closed flux loops, thescale for the electron heating of those high closed loops, and (3) the timescale for iontzation to bring the heavy-ion populations into charge-state equilibrium with the electron temperat re. (In this open-closed temporal wake at the trailing edge, there might also be 
straggling open flux in the temporally developing young streamer-belt region, as depicted in the sketch of Figure 22.) The time evolution of the coronal hole's trailing edge on the moving photosphere corresponds to a spatial evolution on the Sun, which maps to a time evolution in the solar wind for a spacecraft at $1 \mathrm{AU}$. Estimates of the three timescales in the photospheric flow from coronal-hole morphology to streamer-belt morphology are the following. First, the timesc le for the formation of high closed loops in the newly created streamer belt may be obtained from flux-replacement (recycling) estimates: these estimates vary from 1.4 - $3 \mathrm{hr}$ [Close et al., 104_2005] to $40 \mathrm{hr}$ [Schrijver et al., 1997; Schrijver et al., 2007]. Second, the timescale for hea $1 \mathrm{ng}$ ge high hot (1-2 MK) closed loops is in the range of 1 - $6 \mathrm{hr}$ [Sheeley, 1980; Kopp et al., 1985; Viall and Klimchuk, 2011; Reale, 2014]. Third, the timescale for carbon and oxygen to reach $\mathrm{dg}$-state equilibria by progressive ionization in the hot (1-2 MK) high loops is in the range or $\mathrm{s}$ (for $\mathrm{n}_{\mathrm{e}}=10^{8} \mathrm{~cm}^{-3}$ ) to $10^{6} \mathrm{~s}$ (for $\mathrm{n}_{\mathrm{e}}=10^{6} \mathrm{~cm}^{-3}$ ) (cf. Fig. 1 of Smith and Hughes [2010] Or Fig. 3 of Landi et al. [2012]). Taking the geometric means of each of those three timescles yields a total time of $7.5 \mathrm{hr}+2.4 \mathrm{hr}+27 \mathrm{hr}=37 \mathrm{hr}$ for the creation, heating, and equilibration of the high loops in the eastward trailing-edge wake of the coronal hole. Using the results Chappings of the Earth's solar-wind-origin footpoint on the Sun with the WangSheertarge model [Arge et al., 2003] (data courtesy of Nick Arge, 2009) for the years 20042008 orovsky [2016] observed the speed of the Earth's footpoint across the floors of coronal holes to be $0.15 \mathrm{~km} / \mathrm{s}$ to $1 \mathrm{~km} / \mathrm{s}$; the statistical value of $0.51 \mathrm{~km} / \mathrm{s}$ was taken to be a general estimathe speed of the Earth's solar-wind-origin footpoint on the floors of coronal holes. The sogr-yind-origin footpoint speed is probably slower than $0.51 \mathrm{~km} / \mathrm{s}$ near the edges of coronal rores owing to the super-radial expansion of the solar wind near the coronal-hole edges. Taking the speed of the footpoint to be half of $0.51 \mathrm{~km} / \mathrm{s}$, the $37-\mathrm{hr}$ high-loop development time would correspond to a transition boundary thickness of $3.4 \times 10^{4} \mathrm{~km}$ on the photosphere, about one supargenule diameter [e.g. Simon and Leighton, 1964; Hagenaar et al., 1997; Srikanth et al., 2000], wirch is about 1/150 times the equatorial circumference of the Sun. In the absence of solar rarefaction away from the Sun, the Earth would cross this transition in the solar wind 
in $\sim 37 \mathrm{hr}$, however, rarefaction in the trailing edge stretches the crossing time. With a rarefaction by about a factor of 1.8 (estimated in Section 4 from the observed magnetic-field-strength profiles for trailing edges), the $1 \mathrm{AU}$ crossing time is almost doubled from $\sim 37 \mathrm{hr}$ to $\sim 66 \mathrm{hr}$. This $66 \mathrm{hr}$ value is on the order of the thickness of the observed trailing edges at $1 \mathrm{AU}$ and is on the order the thescales seen at $1 \mathrm{AU}$ for the transition in the heavy-ion charge state of the solar wind fom gronal-hole values to streamer-belt values (e.g. Geiss et al. [1995], Zurbuchen et al. [1999] andFigure 14).

It would be interesting to determine whether this wake-like high-loop transition can be image (on the eastward trailing-edge boundaries of coronal-hole extensions relative to the sharper westward leading-edge boundaries. The estimated width of the trailing-edge transition is about 1 . rgranule diameter.

Trapping the close-together solar-wind boundaries in the leading-edge stream interface to the leading edge of a coronal hole and mapping the more-diffuse solar-wind boundaries in the trailing edge stream interface to the trailing edge of a coronal hole with Solar Probe Plus could be a hum for understanding the reconnection dynamics and boundary thicknesses on the two corona edges. Solar Probe Plus will not have ion-charge-state measurements to measure hot-loop plasma injected into open field lines, but perhaps electron-distribution-function mear ments can be used in their place. Separate mappings for isolated coronal holes versus equatorward extensions would be even more powerful.

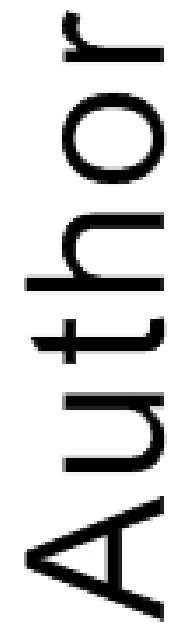




\section{Summary}

The findings of this study are as follows:

(1). 54 high-speed-stream trailing edges at 1 AU were collected in the years 1998-2008 and analyzed. For each trailing edge, five signatures were recorded: the initial velocity drop at the enor the high-speed stream, the upward jump in the $\mathrm{C}^{6+} / \mathrm{C}^{4+}$ charge-state-density ratio, the upward jum in the $\mathrm{O}^{7+} / \mathrm{O}^{6+}$ charge-state ratio, the downward jump of the proton specific entropy $\mathrm{S}_{\mathrm{p}}$, and the hend in the declining velocity temporal profile.

12) The observed temporal widths of the trailing-edge rarefactions are described by simple oalm tic arguments. The mean value and standard deviation of the temporal durations of the trailing edges in the collection is $89 \pm 34 \mathrm{hr}$.

6) The velocity-bend separates the trailing-edge rarefaction into two parts: the first part (prior to tny velocity-bend) is typically about half as long in in time as the second part (after the velocity-bend).

(4). A measure of plasma compression and rarefaction was developed that uses the measuredentations of current sheets in the solar-wind plasma. The current-sheet orientations change whe magnetic structure of the solar-wind plasma is flattened in a compression or stretcrodin a rarefaction.

-T.The leading-edge and trailing-edge stream interfaces are not symmetric; specifically, signatures of the leading-edge (CIR) stream interface occur approximately simultaneously where stream-interface signatures in the trailing-edge rarefaction are temporally separated. Compr sstop versus rarefaction is not enough to account for the leading-edge versus trailingedge timing differences.

(6). The velocity-bend was taken as the best signature to locate the trailing-edge stream interfane. The velocity-bend provides the clearest signature of the maximum of the trailing-edge rarefaction gauged by the magnetic-field strength, the orientation of the plasma current sheets, and the imtensity of the electron strahl and the velocity bend is closest to the maximum of the negativorticity in the trailing edge. 
(7). 1-D computer simulations indicate that the velocity-bend is the collision point of the pressure-driven expansions of the slow streamer-belt-origin wind and the fast coronal-hole-origin wind in the trailing-edge rarefaction.

(8). The downward jump of the proton specific entropy $S_{p}$ in the trailing edge usually occursthear the velocity-bend.

(9). The upward jumps in $\mathrm{C}^{6+} / \mathrm{C}^{4+}$ and $\mathrm{O}^{7+} / \mathrm{O}^{6+}$ tend to occur prior to the velocity bend and pripr to the downward jump of $S_{p}$.

910. The outward imbalance of the Alfvenic fluctuations has a high value in the highspeed calms: the value decays strongly from the high-speed stream to then velocity bend and the decay rate is slow thereafter. The variation in the outward imbalance is owed to a variation in the amsue of outward fluctuations.

(IT) At what appears to be the beginning of the trailing-edge rarefaction, there is an initial velocity drop that terminates the high-speed stream. However, although the velocity does not shdw a decrease, there are other signatures before the initial velocity drop (magnetic-field strength fvenicity, current-sheet orientations) that imply that the trailing-edge rarefaction begins milo this initial velocity drop.

12). It was speculated that the origins of the observed differences of leading-edge stream interfors and trailing-edge stream interfaces at $1 \mathrm{AU}$ might originate from differences in the leading and trailing edges of coronal holes on the Sun. These differences on the Sun might be owed the rigid rotation of coronal holes versus the differential rotation of the photosphere and might ivoye the growth time, the heating time, and the charge-state-equilibration time for high closed loops in the eastern trailing-edge wake of the coronal hole.

A sketch organizing the chronology at 1 AU of the major signatures in the high-speedstreamrrailing edge appears in the five panels of Figure 23. The four vertical dashed lines in the panels he occurrence times of (a) the leading-edge (CIR) stream interface, (b) the initial velocity urop, (c) the velocity-bend in the trailing-edge rarefaction, and (d) the termination of the trailin ge. The initial velocity drop (b) is nominally taken as the start of the trailing-edge 
rarefaction, but there is evidence indicating that the rarefaction might start sooner. The velocitybend (c) is taken as the location of the trailing-edge stream interface. In the top panel the solarwind velocity is sketched. Regions identified as slow wind and as fast wind are labeled, with the fast-wind interval being the high-speed stream. The solar-wind velocity rises across the CIR, takinglabout one day to go from low speeds to high speeds. The locations of the initial velocity drop (land the velocity-bend (c) are both obtained from an examination of the temporal profile of the selarwind velocity. The termination (d) of the trailing edge is taken to be the point where the solr-wind velocity becomes constant or begins to rise after the long-duration decline in speed. (h) second panel of Figure 23 the heavy-ion charge-state-density ratios $\mathrm{C}^{6+} / \mathrm{C}^{4+}$ and $\mathrm{O}^{7+} / \mathrm{O}^{6+}$ are sketched along with the proton specific entropy $\mathrm{S}_{\mathrm{p}}$. All three quantities change rapidly 2 he CIR stream interface (a), with $\mathrm{C}^{6+} / \mathrm{C}^{4+}$ and $\mathrm{O}^{7+} / \mathrm{O}^{6+}$ making the downward transition nom streamer-belt-origin plasma to coronal-hole-origin plasma and $S_{p}$ making the upward transition from streamer-belt-origin to coronal-hole-origin plasma. $\mathrm{C}^{6+} / \mathrm{C}^{4+}, \mathrm{O}^{7+} / \mathrm{O}^{6+}$, and $S_{p}$ beg to make the opposite transitions at the initial velocity drop (b) and complete their transitionat the terminator $(\mathrm{d})$. The tendency in these long transitions is that $\mathrm{C}^{6+} / \mathrm{C}^{4+}$ and $\mathrm{O}^{7+} / \mathrm{O}^{6}$ exhibit a sudden jump upward in that long transition before $\mathrm{S}_{\mathrm{p}}$ exhibits a sudden down jump. (The upward jump in $\mathrm{C}^{6+} / \mathrm{C}^{4+}$ during the long transition can be seen in the green curvent the third panel of Figure 7 at the time of the zero epoch; note that the upward jump in $\mathrm{C}^{6+} / \mathrm{C}^{4+}$ is not as sudden as the drop in $\mathrm{S}_{\mathrm{p}}$, which is the blue curve in the second panel of Figure 7.) $\mathrm{Th}^{6+} \mathrm{C}^{4+}$ and $\mathrm{O}^{7+} / \mathrm{O}^{6+}$ sudden upward jumps occur between the initital velocity drop (b) and the verycity-bend (c); the sudden downward jump of $S_{p}$ occurs near the time of the velocitybend (c). In the third panel of Figure 23 the outward Alfvenic imbalance of the solar wind fluctua ions is sketched. The transition from weakly Alfvenic slow wind to highly Alfvenic fast wind_commences at the CIR stream interface (a) and ends at the end of the CIR. The Alfvenic imbala the high-speed stream begins to decline before the initial velocity drop (b), and the rate of the becomes more rapid after the initial velocity drop. The decline in outward imbalore is almost complete at the time of the velocity-bend (c) and it declines very slowly 
thereafter. In the fourth panel of Figure 23 the magnetic-field strength of the solar wind is sketched. The field-strength undergoes a compression in the CIR that peaks at the CIR stream interface (a). The field strength is constant through some of the high-speed stream, but it begins to decline prior to the time of the initial velocity drop (b). The magnetic-field strength is a minimum at the velocity-bend (c) and increases slowly thereafter. Prior to the initial velocity drop (2, the onset of the temporal decrease of the magnetic-field strength, the onset of the outward_Llfvenic imbalance, and other signatures (e.g. the current-sheet orientation, the transve flow velocity, and the outward Alfvenicity) provide indications that the trailing-edge rarefac 7 ay begin prior to the initial velocity drop. In the bottom panel of Figure 23 the mean value of the out-of-ecliptic angle $\theta_{\text {out-of-ecliptic }}$ of the current-sheet normal vectors in the solar wind p is sketched. The angle being reduced in the CIR indicates compression of the solarwind plasmy and the angle being increased in the trailing edge indicates rarefaction of the solarwind plasma; the compression peaks at the CIR stream interface (a) and the rarefaction peaks at the traing-edge stream interface (c).

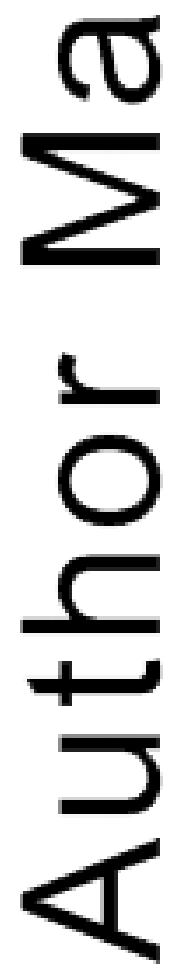




\section{Appendix A: Compression and Rarefaction of Magnetic Structure}

The microscale magnetic structure of the solar wind can be described as magnetic flux tubes bounded by strong current sheets [McCracken and Ness, 1966; Mariani et al., 1983; Bruno et al., 2001, 2004; Borovsky, 2008; D’Amicis et al., 2012; Bruno and Carbone, 2013]. The origin of the strong current sheets (and the flux tubes) may be the Sun [Borovsky, 2008; Neugebauer

and Gicaldne, 2010; Owens et al., 2011] or the flux tubes and current sheets may be generated locally in the solar-wind plasma [Vasquez and Hollweg, 1999; Tsurutani and Ho, 1999; Greco et al., 200. Zhdankin et al., 2012]. The flux tubes are aligned approximately along the Parker spiral [org sky, 2010] and at 1 AU the normal vectors to the current sheets bounding the flux tubes are approximately perpendicular to the Parker spiral [cf. Fig. 8 of Borovsky 2008] and quasi-i ro ic about the Parker-spiral [cf. Fig. 7 of Borovsky 2008]. The quasi-isotropy of the current-sney orientations implies that at $1 \mathrm{AU}$ the flux-tube cross sections are approximately round. (More accurately, the flux-tube cross sections are in the form of a Voronoi pattern with an approx mately equal aspect ratio in the two directions normal to the flux-tube axes (cf. Fig. 1b of Borovs [2008] or Fig. 25 of Borovsky [2016].) An example round flux tube is depicted in the top of Figme 24. A series of spacecraft trajectories that pierce through the round (cylindrical) flux whe are depicted as the blue arrows; where each trajectory crosses through the front boundart of the flux tube the normal vector to the current sheet is denoted with a red vector. The orientation of the current-sheet normal vectors has a range of values from vectors parallel to the ecliptidnlane (for a spacecraft piercing the center of the tube) to vectors strongly out of the ecliptic prane (for piercings far from the center of the tube).

Oirdirectional compression will squash (flatten) the magnetic structure of the solar wind in the Cirection of the compression while leaving the other direction unchanged: such a CIR-like comprassion (by a 2-to1 factor) is depicted in the middle of Figure 24. Again, a series of spacectumectories is depicted as the blue arrows in the sketch and the normal vectors to the currentsinet at the points of piercing are again denoted with red vectors. Note that for the compress d flux tube the normal vectors tend to be focused toward the ecliptic plane relative to 
the normal vectors for the uncompressed round flux tube.

Unidirectional expansion (rarefaction) will stretch the magnetic structure of the solar wind in the direction of the expansion: such a trailing-edge-like rarefaction (by a factor of 2-to-1) is depicted in the bottom of Figure 24. And again, a series of spacecraft trajectories is depicted as the brue arrows and the normal vectors to the current sheet at the points of piercing are again denote with red vectors. Note that for the rarefacted flux tube the normal vectors tend to be turned awav from the ecliptic plane relative to the normal vectors for the uncompressed round flux tub

For llipsoidal round, compressed, and rarefacted flux tubes, the distribution of currentsheet normal vectors that a spacecraft would measure can be calculated numerically. For each norma $\left(n_{r}, n_{t}, n_{n}\right)$, the out-of-ecliptic angle $\theta_{\text {out-of-eclpitic }}=\operatorname{invtan}\left(\left|n_{n}\right| /\left(n_{r}{ }^{2}+n_{t}^{2}\right)^{1 / 2}\right)$ can be calculated. The mean angle of the normal vector out of the ecliptic plane for such numerically calculated distributions is plotted in Figure 25 as a function of the degree of compression or rarefaction: the red curve is the mean angle for compressions and the blue curve is the mean angle fofactions. As seen in Figure 25, compression of the flux tubes reduces the mean angle (turn the normal vectors toward the ecliptic plane) and rarefaction of the flux tubes increast the mean angle (turns the normal vectors away from the ecliptic plane), as depicted in Figu

Note that locally, the axes of the flux tubes of the solar wind are not perfectly aligned with tharker spiral, rather there is a scatter of orientations about the Parker-spiral direction [cf. Fig. 18 OTprovsky, 2010]. At 1 AU the spread in flux tube orientations out of the ecliptic plane had an average half angle of $38^{\circ}$ for all solar wind in the years 1998-2006. The tilting of flux tubes $d$ of the ecliptic plane will reduce high values of the out-of-ecliptic angles $\theta_{\text {out-of-ecliptic }}$ of

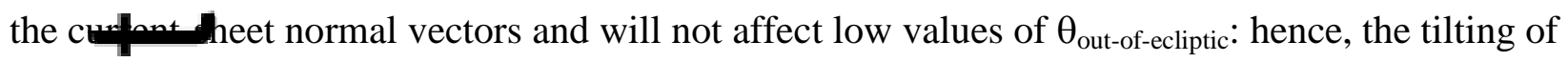
the flux tubgs will reduce the average value of $\theta_{\text {out-of-ecliptic. }}$

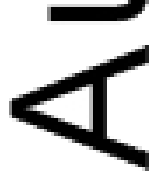


Acknowledgements. The authors wish to thank Roberto Bruno, Len Fisk, Enrico Landi, Pete Riley, Ruth Skoug, John Steinberg, and Dan Welling for helpful conversations. This work was supported at the Space Science Institute by the NSF Solar-Terrestrial Program via grant AGS1261659 , by the NASA Heliophysics LWS TRT program via grants NNX14AN90G and NNX16AB75G, and by the NASA Heliophysics Guest Investigator Program via grant NNX14AC1.5G and supported at the University of Michigan by the NASA Geospace SR\&T progran via grant NNX12AD29G. ENLIL simulations were run at the Community Coordinated Modelig Genter (CCMC) at NASA/Goddard Space Flight Center. All spacecraft data is availab w the NASA data servers http://cdaweb.gsfc.nasa.gov, http://omniweb.gsfc.nasa.gov, and . httn//omniweb.gsfc.nasa.gov/coho/ and from the ACE data server http://vww.srl.caltech.edu/ACE/ASC/. ENLIL simulation outputs are available at the CCMC server http.trccmc.gsfc.nasa.gov.

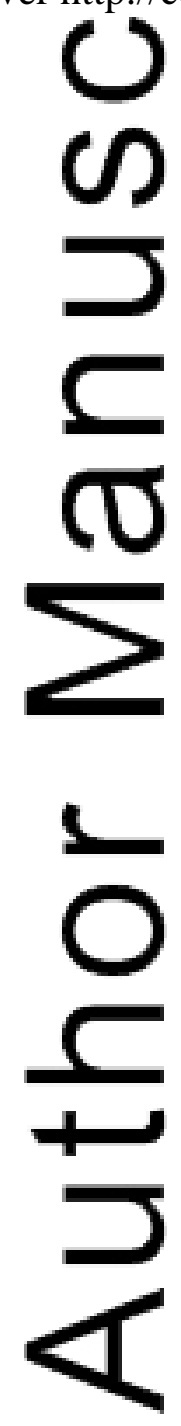




\section{References}

Adams, W. M., Differential rotation of photospheric magnetic fields associated with coronal holes, Solar Phys., 47, 601, 1976.

Arge, C.N., D. Odstrcil, V.J. Pizzo, and L.R. Mayer, Improved method for specifying solar wind speed near the sun. In: The Tenth International Solar Wind Conference. AIP, Melville, NY, pp.190-193, 2003.

Asbride, R., S. J. Bame, W. C. Feldman, and M. D. Montgomery, Helium and hydrogen ve ait differences in the solar wind, J. Geophys. Res., 81, 2719, 1976.

Baker, D L . van Driel-Gesztelyi, and G. R. R. Attril, Evidence for interchange reconnection between a coronal hole and an adjacent emerging flux region, Astron. Nachr., 328, 773, 2007.

Belche J. V., and L. Davis, Large-amplitude Alfven waves in the interplanetary medium, 2, $J$. Geosh. Res., 76, 3534, 1971.

Borovsly E., The flux-tube texture of the solar wind: Strands of the magnetic carpet at 1 AU?, J. Gophys. Res., 113, A08110, 2008.

Borovs $\mathrm{k}$ E., On the variations of the solar-wind magnetic field about the Parker-spiral directio, J. Geophys. Res., 115, A09101, 2010.

Borovsky, J. E., Plasma structure of the coronal-hole solar wind: Origins and Evolution, su mitted to J. Geophys. Res., 121, doi:10.1002/2016JA022686, in press 2016.

Borovs E., and M. H. Denton, The differences between CME-driven storms and CIR-driven stormo Geophys. Res., 111, A07S08, 2006.

Borovs J J E., and M. H. Denton, On the Heating of the Outer Radiation Belt to Produce High Fluxes of Relativistic Electrons: Measured Heating Rates for High-Speed-Stream-Driven Socms, J. Geophys. Res., 115, A12206, 2010a.

Borovsky. E., and M. H. Denton, The magnetic field at geosynchronous orbit during highspectrom-driven storms: Connections to the solar wind, the plasma sheet, and the outer electron radiation belt, J. Geophys. Res., 115, A08217, 2010b.

Borovsky, J. E., and M. H. Denton, Solar-wind turbulence and shear: A superposed-epoch anarysis of corotating interaction regions at 1 AU, J. Geophys. Res., 115, A10101, 2010c.

Borovs y, E., and M. H. Denton, The differences between storms driven by helmet-streamer CIRs a d storms driven by pseudostreamer CIRs, J. Geophys. Res., 118, 5506, 2013.

Borovskv_I E., and S. P. Gary, How important are the alpha-proton relative drift and the elc ctron heat flux for the proton heating of the solar wind in the inner heliosphere?, $J$. Geophys. Res., 119, 5210, 2014.

Borrim, O., J. T. Gosling, S. J. Bame, W. C. Feldman, and J. M. Wilcox, Solar wind helium and hydon structure near the heliospheric current sheet: A signal of coronal streamers at 1 AU.J.Jeophys. Res., 86, 4565, 1981.

Bruno, R., and B. Bavassano, Origin of low cross-helicity regions in the inner solar wind, $J$. Cop hys. Res., 96, 7841, 1991. 
Bruno, R., and V. Carbone, The solar wind as a turbulence laboratory, Living Rev. Solar Phys. 10, 2, http://www.livingreview.org/lrsp-2013-2, 2013.

Bruno, R., V. Carbone, P. Veltri, E. Pietropaolo, and B. Bavassano, Identifying intermittency events in the solar wind, Planet. Space Sci., 49, 1201, 2001.

Bruno, R., V. Carbone, L. Primavera, F. Malara, L. Sorriso-Valvo, B. Bavassano, and P. Veltri, On the probability distribution function of small-scale interplanetary magnetic field fructuations, Ann. Geophys., 22, 3751, 2004.

Burlag L.F., Interplanetary stream interfaces, J. Geophys. Res., 25, 3717, 1974.

Burlag and N. F. Ness, Tangential discontinuities in the solar wind, Solar Phys., 9, 467, 1960

Burlag, L. F., W. H. Mish, and Y. C. Whang, Coalescence of recurrent streams of different sizes andramplitudes, J. Geophys. Res., 95, 4247, 1990.

Burton M. E., M. Neugebauer, N. U. Crooker, R. von Steiger, and E. J. Smith, Identification of tran edge stream interfaces: A comparison of Ulysses plasma and composition measments, J. Geophys. Res., 104, 9925, 1999.

Carovibor. R. L., and G. L. Siscoe, Corotating structure in the solar wind, Solar Phys., 8, 401, 1969

Close, R. M , C. E. Parnell, D. W. Longcope, and E. R. Priest, Recycling of the solar corona's magnetic field, Astrophys. J., 612, L81, 2004.

Close, r. Rr., C. E., Parnell, D. W. Longcope, and E. R. Priest, Coronal flux recycling times, Sold 231, 45, 2005.

Crooker U. U., and J. T. Gosling, CIR morphology, turbulence, discontinuities, and energetic patidle, Space Sci. Rev., 89, 179, 1999.

Crooker, N. U., and R. L. McPherron, Coincidence of composition and speed boundaries of the siow solar wind, J. Geophys. Res., 117, A09104, 2012.

Crooker U., G. L. Siscoe, S. Shodhan, D. F. Webb, J. T. Gosling, and E. J. Smith, Multiple movpmeric current sheets and coronal streamer belt dynamics, J. Geophys. Res., 98, 9371, 1993.

Crooker, N. U., M. E. Burton, J. L. Phillips, E. J. Smith, and A. Balogh, Heliospheric plasma sheets as small-scale transients, J. Geophys. Res., 101, 2426, 1996.

Crooke U., S. Shokhan, R. J. Forsyth, M. E. Burton, J. T. Gosling, R. J. Fitzenreiter, and R. P. Lep ing, Transient aspects of stream interface signatures, AIP Conf. Proc., 471, 597, 1999

Crook N. U., S.W. Kahler, J. T.Gosling, D. E. Larson, R. P. Lepping, E. J. Smith, and J. De Keyser, Scales of heliospheric current sheet coherence between 1 and 5 AU, J. Geophys. Ru., 106, 15963, 2001.

CrookeU., E. M. Appleton, N. A. Schwadron, and M. J. Owens, Suprathermal electron flux peaks 2 stream interfaces: Signature of solar wind dynamics or tracer for open magnetic flux transport on the Sun?, J. Geophys. Res., 115, A11101, 2010.

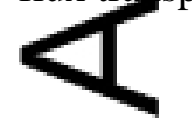


D’Amicis, R., G. Consolini, B. Bavassano, and R. Bruno, Conditioned analysis of high-latitude solar wind intermittency, Astrophys. J., 755, 63, 2012.

Denton, M. H., J. E. Borovsky, R. M. Skoug, M. F. Thomsen, B. Lavraud, M. G. Henderson, R. L. McPherron, J. C. Zhang and M. W. Liemohn, Geomagnetic storms driven by ICME- and CIR-dominated solar wind, J. Geophys. Res., 111, A07S07, 2006.

Ebert, R. W., M. A. Dayeh, M. I. Desai, and G. M. Mason, Corotating interaction region associated suprathermal helium ion enhancements at $1 \mathrm{AU}$ : Evidence for local acceleration at regmpression region trailing edge, Astrophys. J., 749, 73, 2012.

Elliott, D. McComas, N. A. Schwadron, J. T. Gosling, R. M. Skoug, G. Gloeckler, and T.H_Zurbuchen, An improved expected temperature formula for identifying ICMEs, $J$. Geophys. Res., 110, A04103, 2005.

Feldman, U., K. G. Widing, and H. P. Warren Morphology of the quiet solar upper atmosphere in 1 e $4.10^{4}<\mathrm{T}_{\mathrm{e}}<1.4 \times 10^{6} \mathrm{~K}$ temperature regime, Astrophys. J., 522, 1133, 1999.

Fisk, L_. T. H. Zurbuchen, and N. A. Schwadron, Coronal hole boundaries and their interajons with adjacent regions, Space Sci. Rev., 87, 43, 1999a.

Fisk, A. T. H. Zurbuchen, and N. A. Schwadron, On the coronal magnetic field: Conseayences of large-scale motions, Astrophys. J., 521, 868, 1999 b.

Forsyth, R.J., and E. Marsch, Solar origin and interplanetary evolution of stream interfaces, Space Sci. Rev., 89, 7, 1999.

Freema 1, J. W., and R. E. Lopez, The cold solar wind, J. Geophys. Res., 90, 9885, 1985.

Galvin__. L. M. Kistler, M. A. Popecki, et al., The plasma and suprathermal ion composition

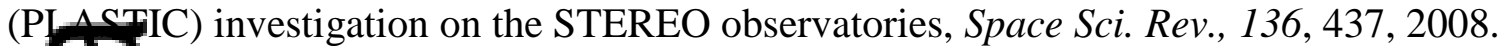

Geiss, G.G Gloeckler, and R. von Steiger, Origin of solar wind from composition data, Space Sci. Rev., 72, 49, 1995.

Gloeciker G., J. Cain, F. M. Ipavich, E. O. Tums, P. Bedini, L. A. Fisk, T. H. Zurbuchen, p. Bacbiter, J. Fischer, R. F. Wimmer-Schweingruber, J. Geiss, and R. Kallenbach, mestrgation of the composition of solar and interstellar matter using solar wind and pickup ion measurements with SWICS and SWIMS on the ACE spacecraft, Space Sci. Rev., 86, 497, 1998.

Gloecker, G., T. H. Zurbuchen, and J. Geiss, Implications of the observed anticorrelation be vee solar wind speed and coronal electron temperature, J. Geophys. Res., 108, 1158, 2063.

Gosling___T., and V. J. Pizzo, Formation and evolution of corotating interaction regions and thcir three dimensional structure, Space Sci. Rev., 89, 21, 1999.

Gosling, J.T., V. Pizzo, and S. J. Bame, Anomalously low proton temperatures in the solar wind fouwing interplanetary shock waves -- Evidence for magnetic bottles, J. Geophys. Res., 78, $2001-1973$.

Gosling_J. J., J. R. Asbridge, S. J. Bame, and W. C. Feldman, Solar wind stream interfaces, J. Geophys. Res., 83, 1401, 1978.

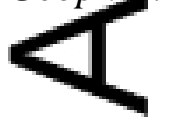


Gosling, J. T., G. Borrini, J. R. Asbridge, S. J. Bame, W. C. Feldman, and R. T. Hansen, Coronal streamers in the solar wind at 1 AU, J. Geophys. Res., 86, 5438, 1981.

Gosling, J. T., D. N. Baker, S. J. Bame, W. C. Feldman, R. D. Zwickl, and E. J. Smith, Bidirectional solar wind electron heat flux events, J. Geophys. Res., 92, 8519, 1987.

Greco, A., W. H. Matthaeus, S. Servidio, P. Chuychai, and P. Dmitruk, Statistical analysis of discontinuities in solar wind ACE data and comparison with intermittent MHD turbulence, Astropnys. J., 691, L111, 2009.

Hagenar, 4. J., C. J. Schriver, and A. M. Title, The distribution of cell sizes of the solar cham anhere network, Astrophys. J., 481, 998, 1997.

Hiremath_K. M., and M. Hedge, Rotation rates of coronal holes and their probable anchoring depths, Astrophys. J., 763, 137, 2013,

Hirshberg, J., J. R. Asbridge, and D. E. Robbins, The Helium component of solar wind velocity streams J. Geophys. Res., 79, 934, 1974.

Hundharad, A. J., Nonlinear model of high-speed solar wind streams, J. Geophys. Res., 78, $1528+973$.

Intriligt r D. S., J. R. Jokipii, T. S. Horbury, J. M. Intriligator, R. J. Forsyth, H. Kunow, G. Wibherenz, and J. T. Gosling, Processes associated with particle transport in corotating interact on regions and near stream interfaces, J. Geophys. Res., 106, 10625, 2001.

Jian, L., C. T. Russell, J. G. Luhmann, and R. M. Skoug, Properties of stream interactions at one A caurng 1995-2004, Solar Phys., 239, 337, 2006.

Kahler $A$ search for compositional differences in slow solar wind at the leading and trailing edgacos stream interfaces, AIP Conf. Proc., 216, 383, 2010.

Kahler (S. Jibben, and E. E. DeLuca, TRACE observations of changes in coronal hole boundaries, Solar Phys., 262, 135, 2010.

Kasper, C., A. J. Lazarus, J. T. Steinberg, K. W. Ogilvie, and A. Szabo, Physics-based tests to identify the accuracy of solar wind ion measurements: A case study with the Wind Faraday Cups, Geophys. Res., 111, A03105, 2006.

King, J. H., and N. E. Papitashvili, Solar wind spatial scales in and comparisons of hourly Wind and ACE plasma and magnetic field data, J. Geophys. Res., 110, 2104, 2005.

Knetter, 1.,F. M. Neubauer, T. Horbury, and A. Balogh, Four-point discontinuity observations usiggluster magnetic field data: A statistical survey, J. Geophys. Res., 109, A06102, 2004.

Ko, Y.K. C. Raymond, T. H. Zurbuchen, P. Riley, J. M. Raines, and L. Strachan, Abundance variation at the vicinity of an active region and the coronal origin of slow solar wind, As rophys. J., 646, 1275, 2006.

Kopp, R. A. G. Poletto, G. Noci, and M. Bruner, Analysis of loop flows observed on 27 March, Gwo oy the UVSP instrument during the Solar Maximum Mission, Solar Phys., 98, 91, 1905

Krista,L.D., P. T. Gallagher, and D. S. Bloomfield, Short-term evolution of coronal hole boundaries, Astrophys. J. Lett., 731, L26, 2011.

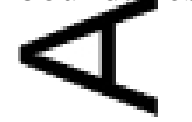


Landi, E., R. L. Alexander, J. R. Gruesbeck, J. A. Gilbert, S. T. Lepri, W. B. Manchester, and T. H. Zurbuchen, Carbon ionization stages as a diagnostic of the solar wind, Astrophys. J., 744, 100, 2012.

Lavraud, B., M. F. Thomsen, J. E. Borovsky, M. H. Denton, and T. I. Pulkkinen, Magnetosphere preconditioning under northward IMF: Evidence from the study of coronal mass ejection and corotating interaction region geoeffectiveness, J. Geophys. Res., 111, A09208, 2006.

Lazarus, A., J. Kasper, A. Szabo, and K. Ogilvie, Solar wind streams and their origins, AIP Proc., 67, ps 187, 2003.

Leppin_ D. M. H. Acuna, L. F. Burlaga, W. M. Farrell, J. A. Slavin, K. H. Schatten, F. Mariani N. F. Ness, F. M. Neubauer, Y. C. Whang, J. B. Byrnes, R. S. Kennon, P. V. Panetta, J. Scheifele, and E. M. Worley, The Wind magnetic field investigation, Space Sci. Rev., TT, 207, 1995.

Leppin s, R.P., C.-C. Wu, and D. B. Berdichevsky, Automatic identification of magnetic cloudlik 1 ons at 1 AU: occurrence rate and other properties, Ann. Geophys., 23, 2687, 2005.

Lin, R.P. A. A. Anderson, S. Ashford, C. Carlson, D. Curtis, R. Ergun, D. Larson, J. McFadden, M.darthy, G. K. Parks, H. Reme, J. M. Bosqued, J. Coutelier, F. Cotin, C. D'uston, K.-P. Wenzel T. R. Sanderson, J. Henrion, J. C. Ronnet, and G. Paschmann, A three-dimensional plasma and energetic particle investigation for the WIND spacecraft, Space Sci. Rev., 71, $125,1995$.

Lucek, E. A., and A. Balogh, The identification and characterization of Alfvenic fluctuations in UE VEcES data at midlatitudes, Astrophys. J., 507, 984, 1998.

Mariani_B. Bavassano, and U. Villante, A statistical study of MHD discontinuities in the inier so ar system: Helios 1 and 2, Solar Phys., 83, 349, 1983.

Marsch, E.,K.-H. Muhlhauser, H. Rosenbauer, R. Schwenn, and F. M. Neubauear, Solar wind howum 1ons: Observations of the Helios solar probes between 0.3 and 1 AU, J. Geophys. Res $87,35,1982$.

McCuman, D. J., S. J. Blame, P. Barker, W. C. Feldman, J. L. Phillips, P. Riley, and J. W. Griffee, Solar Wind Electron Proton Alpha Monitor (SWEPAM) for the Advanced Composition Explorer, Space Sci. Rev. 86, 563, 1998.

McCraeneri, K. G., and N. F. Ness, The collimation of cosmic rays by the interplanetary magnejic field, J. Geophys. Res., 71, 3315, 1966.

McPhe ron R. L., and J. Weygand, The solar wind and geomagnetic activity as a function of time_relative to corotating interaction regions, in Recurrent Magnetic Storms, B. Tsurutani, R. McPherron, W. Gonzalez, G. Lu, J. H. A. Sobral, and N. Goalswamy (eds.), pg. 125, American Geophysical Union, 2006.

McPlurum, R. L., D. N. Baker, and N. U. Crooker, Role of the Russell-McPherron effect in the action of relativistic electrons, J. Atmos. Solar-Terr. Phys., 71, 1032, 2009.

Nakagawa. r., Solar source of the interplanetary planar magnetic structures, Solar Phys., 147, $169,1993$.

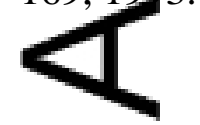


Nakagawa, T., A. Nishida, and T. Saito, Planar magnetic structures in the solar wind, J. Geophys. Res., 94, 11761, 1989.

Nash, A. G., N. R. Sheeley, and Y.-M. Wang, Mechanisms for the rigid rotation of coronal holes, Solar Phys., 117, 359, 1988.

Navarro-Peralta, P., and A. Sanchez-Ibarra, An observational study of coronal hole rotation over the sunspot cycle, Solar Phys., 153, 169, 1994.

Neugetuauer, M., and J. Giacalone, Progress in the study of interplanetary discontinuities, AIP Co (1. Troc., 1216, 194, 2010.

Ogilvie $\mathrm{K}_{\mathrm{K}}$ et al., SWE, a comprehensive plasma instrument for the WIND spacecraft, Space Sci_Rev, 71, 55, 1995.

Owens M. J., R. T. Wicks, and T. S. Horbury, Magnetic discontinuities in the near-Earth solar wintu. Evidence of in-transit turbulence or remnants of coronal structure?, Solar Phys., 269, $41 \boldsymbol{c}, 201$.

Pagel, ., N. U. Crooker, T. H. Zurbuchen, and J. T. Gosling, Correlation of solar wind entyog and oxygen ion charge state ratio, J. Geophys. Res., 109, A01113, 2004.

Pizzo, $\mathbf{V}$, The evolution of corotating stream fronts near the ecliptic plane in the inner solar system1. Two-dimensional fronts, J. Geophys. Res. 94, 8673, 1989.

Pizzo, V. J. The evolution of corotating stream fronts near the ecliptic plane in the inner solar system 2. Three-dimensional tilted-dipole fronts, J. Geophys. Res., 96, 5405, 1991.

Reale, (, Coronal loops: Observations and modeling confined plasmas, Living Rev. Solar Phys., 11 (1) 14.

Richardcan I. G., Low energy ions in co-rotating interaction regions at 1 AU: Evidence for statis icl 1 ion acceleration, Planet. Space Sci., 33, 557, 1985.

Richardson, I. G., and H. V. Cane, Near-Earth interplanetary coronal mass ejections during solar cye 23 (1996-2009): Catalog and summary of properties, Solar Phys., 264, 189, 2010.

Richardso, I. G., D. F. Webb, J. Zhang, D. B. Berdichevsky, D. A. Besecker, J. C. Casper, R. natavira, J. T. Steinberg, B. J. Thompson, C.-C. Wu, and A. N. Zhukov, Major geomagnetic storms (Dst $\leq-100 \mathrm{nT}$ ) generated by corotating interaction regions, J. Geophys. Res., 111, Ac7S09, 2006.

Richter, A.K., and A. H. Luttrell, Superposed epoch analysis of corotating interaction regions at 0.3 and 1.0 AU: A comparative study, J. Geophys. Res., 91, 5873, 1986.

Schrijver. J., Braiding-induced interchange reconnection of the magnetic field and the width of solar.coronal loops, Astrophys. J., 662, L119, 2007.

Schrij、er, C. J., A. M. Title, A. A. van Ballegooijen, H. J. Hagenaar, and R. A. Shine, Sustaining the quiet photospheric network: The balance of emergence, fragmentation merging, and carcenration, Astrophys. J., 487, 424, 1997.

Sheele N. R., Temporal variation of loop structures in the solar atmosphere, Solar Phys., 66, 79.198).

Simon, G. W., and R. B. Leighton, Velocity fields in the solar atmosphere. III. Large-scale motr n, the chromospheric network, and magnetic fields, Astrophys. J., 140, 1120, 1964. 
Simunac, K. D. C., L. M. Kistler, A. B. Galvin, M. A. Lee, M. A. Popecki, C. Farrugia, E. Moebius, L. M. Blush, P. Bochsler, P. Wurz, B. Klecker, R. F. Wimmer-Schweingruber, B. Thompson, J. G. Luhmann, C. T. Russell, and R. A. Howard, In situ observations of solar wind stream interface evolution, Solar Phys., 259, 323, 2009.

Simunac, K. D. C., A. B. Galvin, J. Barry, C. Farrugia, L. M. Kistler, H. Kucharek, M. A. Lee, Y. C.-M. Liu, E. Mobius, and M. A. Popecki, Identifying the ends of high-speed streams near 1 AUwith in situ data from STEREO/PLASTIC, AIP Conf. Proc., 216, 351, 2010.

Siscoe, G., Ind D. Intriligator, Three views of two giant streams: Aligned observations at 1 AU, 4. AU and 5.9 AU, Geophys. Res. Lett., 20, 2267, 1993.

Siscoe_G_. B. Goldstein, and A. J. Lazarus, An East-West asymmetry in the solar wind velocity, J. Geophys. Res., 74, 1759, 1969.

Smith, C. w., M. H. Acuna, L. F. Burlaga, J. L'Heureux, N. F. Ness, and J. Scheifele, The ACE Menetic Fields Experiment, Space Sci. Rev., 86, 611, 1998.

Smith, K. and J. P. Hughes, Ionization equilibrium timescales in collisional plasmas, Astromys. J., 718, 583, 2010..

Solom S C., A. G. Burns, B. A. Emery, M. G. Mlynczak, L. Qian, W. Wang, D. R. Weimer, and Miltberger, Modeling studies of the impact of high-speed streams and corotating interact on regions on the thermosphere-ionosphere, J. Geophys. Res., 117, A00L11, 2012.

Srikanth, R., J. Singh, and K. P. Raju, Distribution of supergranule sizes, Astrophys. J., 534, 100,2000 .

Subranm, S., M. S. Madjarska, and J. G. Doyle, Coronal hole boundaries evolution at small scalac- XRT view. Can small-scale outflows at CHBs be a source of the slow wind?, Astr. Astrophys., 516, A50, 2010.

Tessein, J. A., C. W. Smith, B. J. Vasquez, and R. M. Skoug, Turbulence associated with conating interaction regions at $1 \mathrm{AU}$ : Inertial and dissipation rage magnetic field spectra, $J$. Geoninys. Res., 116, A10104, 2011.

Timany, F., A. S. Krieger, and G. S. Vaiana, The structure and evolution of coronal holes, Solar Phys., 42, 135, 1975.

Tsurutani, B. T., and C. M. Ho, A review of discontinuities and Alfven waves in interplanetary space. ULYSSES results, Rev. Geophys., 37, 517, 1999.

Tsurutanin. T., W. D. Gonzalez, A. L. C. Gonzalez, F. Tang, J. K. Arballo, and M. Okada, Introlbetary origin of geomagnetic activity in the declining phase of the solar cycle, $J$. Geonhus. Res., 100, 21717, 1995.

Tsurut,ni, B., R. McPherron, W. Gonzalez, G. Lu, J. H. A. Sobral, and N. Goalswamy (eds.), Recurrent Magnetic Storms, American Geophysical Union, 2006.

Tu, C.1., and E. Marsch, MHD structures, waves and turbulence in the solar wind, Space Sci. $\operatorname{Re} 72$ 1, 1995.

Vasquez. B J., and J. V. Hollweg, Formation of pressure-balanced structures and fast waves from nonlinear Alfven waves, J. Geophys. Res., 104, 4681, 1999.

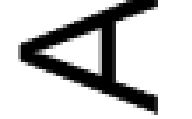


Viall, N. M., and J. A. Klimchuk, Patterns of nanoflare storm heating exhibited by an active region observed with Solar Dynamics Observatory/Atmosphere Imaging Assembly, Astrophys. J., 738, 24, 2011.

Wang, Y.-M., and N. R. Sheeley, Footpoint switching and the evolution of coronal holes, Astrophys. J., 612, 1196, 2004.

Wimmer-Schweingruber, R. R., R. von Steiger, and R. Paerli, Solar wind stream interfaces in cơveratng interaction regions: SWICS/Ulysses results, J. Geophys. Res., 102, 17407, 1997.

$\mathrm{Xu}, \mathrm{F}$. amy J. E. Borovsky, A new 4-plasma categorization scheme for the solar wind, $J$. Ge ${ }_{\text {R }}$ es., 120, 70, 2015.

Yang LZZhang, T. Li, and Y. Liu, SDO observations of magnetic reconnection at coronal hol boundaries, Astrophys. J. Lett., 732, L7, 2011.

Zhao, E., T. H. Zurbuchen, and L. A. Fisk, Global distribution of the solar wind during solar cyce 23) ACE observations, Geophys. Res. Lett., 36, L14104, 2009.

Zhdank ., S. Boldyrev, J. Mason, and J. C. Perez, Magnetic discontinuities in magneghydrodynamic turbulence and in the solar wind, Phys. Rev. Lett., 108, 175004, 2012.

Zirker, $U_{\text {B }}$, Coronal holes and high-speed wind streams, Rev. Geophys. Space Phys., 15, 257, 1977

Zurbuchen, r. H., S. Hefti, L. A. Fisk, G. Gloeckler, and R. von Steiger, The transition between fast and slow solar wind from composition data, Space Sci. Rev., 87, 353, 1999.

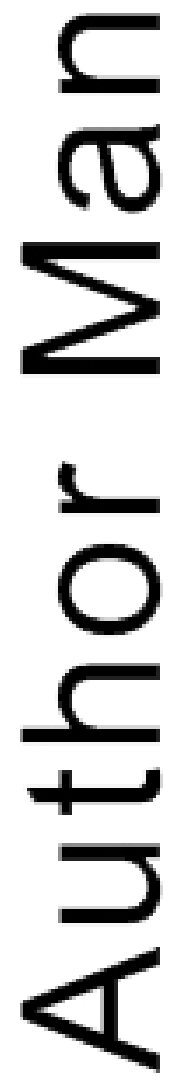


Table 1 . The collection of 54 high-speed-stream trailing edges. The 1 st column is the event number, 2nd column the year, 3rd column the time of the initial velocity drop, 4th column the time of the velocity-bend, 5th column the time of the velocity-decline termination, 6th column the speed of the fast wind in the high-speed stream, 7th column the speed of the solar wind at the time of termination, 8th column the type of streamer belt [HS = helmet streamer $(1$ sector reversal), PS $=$ pseudostreamer $($ no sector reversal), DSR $=$ double sector reversal $(2$ sector reversa.s)], and the 9th column is the total time duration of the rarefaction (termination time minus me initial velocity drop).

\begin{tabular}{|c|c|c|c|c|c|c|c|c|}
\hline $\begin{array}{c}\text { Event } \\
\# .\end{array}$ & Ven & $\begin{array}{l}\text { nitial velocity drop } \\
\text { [day of year] }\end{array}$ & $\begin{array}{l}\text { Velocity-bend } \\
\text { [day of year] }\end{array}$ & $\begin{array}{c}\text { Termination } \\
\text { [day of year] }\end{array}$ & $\begin{array}{c}\mathrm{v}_{\text {fast }} \\
{[\mathrm{km} / \mathrm{s}]}\end{array}$ & $\begin{array}{c}\mathrm{v}_{\text {slow }} \\
{[\mathrm{km} / \mathrm{s}]}\end{array}$ & Type & $\begin{array}{c}\Delta \mathrm{t}_{\text {total }} \\
{[\mathrm{hr}]}\end{array}$ \\
\hline 1 & & 303.29 & 303.70 & 305.80 & 578 & 367 & HS & 60.1 \\
\hline 2 & & 298.88 & 299.76 & $?$ & 557 & $?$ & HS & $?$ \\
\hline 3 & & 30.33 & 33.12 & 36.55 & $?$ & 371 & HS & 149.3 \\
\hline 4 & & 345.41 & 348.79 & 351.13 & 643 & 312 & HS & 137.3 \\
\hline 5 & & 201.00 & 201.75 & 203.41 & 577 & 361 & $\mathrm{HS}$ & 57.9 \\
\hline 6 & & 66.21 & 67.58 & 70.46 & 615 & 358 & PS & 102.1 \\
\hline 7 & & 92.59 & 95.00 & 96.62 & 667 & 328 & PS & 96.7 \\
\hline 8 & & 135.38 & 137.87 & 138.62 & 697 & 404 & $\mathrm{HS}$ & 77.8 \\
\hline 9 & & 162.63 & 163.42 & 164.50 & 670 & 394 & $\mathrm{HS}$ & 44.9 \\
\hline 10 & & 250.04 & 250.41 & 251.50 & 560 & 376 & PS & 35.0 \\
\hline 11 & $\overline{03}$ & 255.96 & 256.75 & 258.50 & 601 & 351 & HS & 61.0 \\
\hline 12 & 03 & 268.79 & 270.46 & 273.04 & 691 & 293 & HS & 102.0 \\
\hline 13 & & 349.50 & 350.87 & 353.96 & 750 & 304 & $\mathrm{HS}$ & 107.2 \\
\hline 14 & & 64.50 & 64.79 & 67.92 & 579 & 318 & HS & 82.0 \\
\hline 15 & & 336.46 & 337.75 & 340.17 & 655 & 295 & $\mathrm{HS}$ & 89.0 \\
\hline 16 & & 353.84 & 354.84 & 356.09 & 585 & 348 & HS & 54.0 \\
\hline 17 & & 24.00 & 25.25 & 27.38 & 667 & 323 & $\mathrm{HS}$ & 81.2 \\
\hline 18 & $\overline{\sigma o s}$ & 41.63 & $?$ & 44.96 & 717 & 361 & HS & 79.9 \\
\hline 19 & 2005 & 62.17 & 62.96 & 64.08 & 622 & 377 & $\mathrm{HS}$ & 46.0 \\
\hline 20 & 2005 & 69.58 & 71.46 & 72.79 & 665 & 323 & HS & 77.1 \\
\hline 21 & & 96.29 & 97.38 & 99.37 & 593 & 318 & DBL & 73.8 \\
\hline 22 & & 177.25 & 178.20 & 179.00 & 592 & 374 & HS & 42.0 \\
\hline 23 & & $?$ & 230.63 & 233.46 & 669 & 371 & HS & $?$ \\
\hline 24 & 05 & 284.25 & 285.00 & 288.42 & 619 & 298 & HS & 100.1 \\
\hline 25 & 2005 & 311.58 & 312.46 & 315.00 & 626 & 348 & $\mathrm{HS}$ & 82.1 \\
\hline $26=$ & & 338.08 & 339.95 & 343.67 & 675 & 263 & $\mathrm{HS}$ & 133.9 \\
\hline $27=$ & 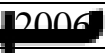 & 81.33 & 82.75 & 85.46 & 613 & 338 & $\mathrm{HS}$ & 99.2 \\
\hline 28 & 2006 & 100.58 & 102.21 & 102.71 & 670 & 382 & HS & 51.0 \\
\hline 29 & 2006 & 113.62 & 114.50 & 118.00 & 547 & 323 & $?$ & 105.1 \\
\hline 30 & 2000 & 134.04 & 135.25 & 137.29 & 585 & 319 & HS & 78.1 \\
\hline 31 & & 154.54 & 155.00 & 157.08 & 570 & 323 & HS & 61.0 \\
\hline 32 & & 187.95 & $?$ & 190.79 & 567 & 330 & HS & 68.2 \\
\hline
\end{tabular}




\begin{tabular}{|c|c|c|c|c|c|c|c|c|}
\hline 33 & 2006 & 215.75 & 216.67 & 218.96 & 527 & 331 & $\mathrm{HS}$ & 77.0 \\
\hline 34 & 2006 & 303.63 & 304.41 & 307.38 & 559 & 333 & $\mathrm{HS}$ & 90.0 \\
\hline 35 & 2006 & 316.38 & 317.62 & 319.63 & 590 & 347 & $\mathrm{HS}$ & 77.9 \\
\hline 36 & 2006 & $?$ & 361.04 & 362.50 & $?$ & 328 & HS & $?$ \\
\hline 37 & 2007 & 46.79 & 49.87 & 55.26 & 684 & 268 & $\mathrm{HS}$ & 203.4 \\
\hline 38 & 2007 & 60.96 & 61.96 & 62.71 & 600 & 340 & $\mathrm{HS}$ & 42.0 \\
\hline 39 & | & 66.75 & 68.25 & 70.25 & 625 & 298 & HS & 84.0 \\
\hline 40 & 2007 & 93.87 & 95.46 & 98.84 & 583 & 295 & $\mathrm{HS}$ & 119.2 \\
\hline 41 & (2007) & 102.55 & 103.83 & 106.87 & 524 & 310 & $\mathrm{HS}$ & 103.8 \\
\hline 42 & 2007 & 120.50 & 122.71 & 126.88 & 646 & 266 & HS & 153.0 \\
\hline $43^{-}$ & $200 T$ & 129.67 & 130.08 & 133.87 & 587 & 299 & HS & 100.9 \\
\hline 44 & 2007 & 140.29 & $?$ & 142.71 & 620 & 420 & $\mathrm{HS}$ & 58.0 \\
\hline 45 & 2007 & 148.17 & 148.75 & 151.54 & 629 & 305 & HS & 81.0 \\
\hline 46 & 2007 & 293.92 & 294.67 & 298.25 & 664 & 340 & PS & 104.0 \\
\hline 47 & & 330.33 & 331.71 & 334.67 & 631 & 329 & $\mathrm{HS}$ & 104.2 \\
\hline 48 & 2001 & 347.83 & $?$ & 350.80 & 557 & 350 & $\mathrm{HS}$ & 71.2 \\
\hline 49 & & 357.16 & 358.25 & 360.29 & 601 & 333 & $\mathrm{HS}$ & 75.2 \\
\hline 50 & קمصد & 35.25 & 36.08 & 38.54 & 617 & 336 & HS & 79.1 \\
\hline 51 & 2008 & 62.04 & 64.17 & 67.88 & 754 & 316 & $\mathrm{HS}$ & 140.2 \\
\hline 52 & 2008 & 127.32 & $?$ & 133.79 & 644 & 304 & PS & 155.3 \\
\hline 53 & 2008 & 197.62 & 200.82 & 202.46 & 647 & 288 & HS & 116.2 \\
\hline 54 & בסמת & 206.34 & 206.71 & 209.17 & 646 & 341 & PS & 68.0 \\
\hline
\end{tabular}
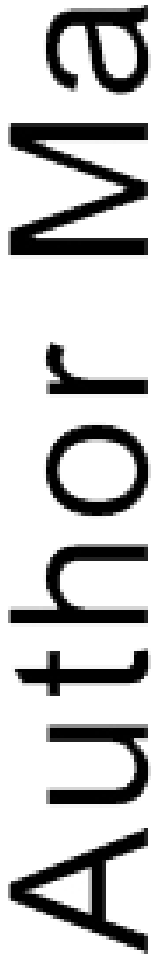

This article is protected by copyright. All rights reserved. 

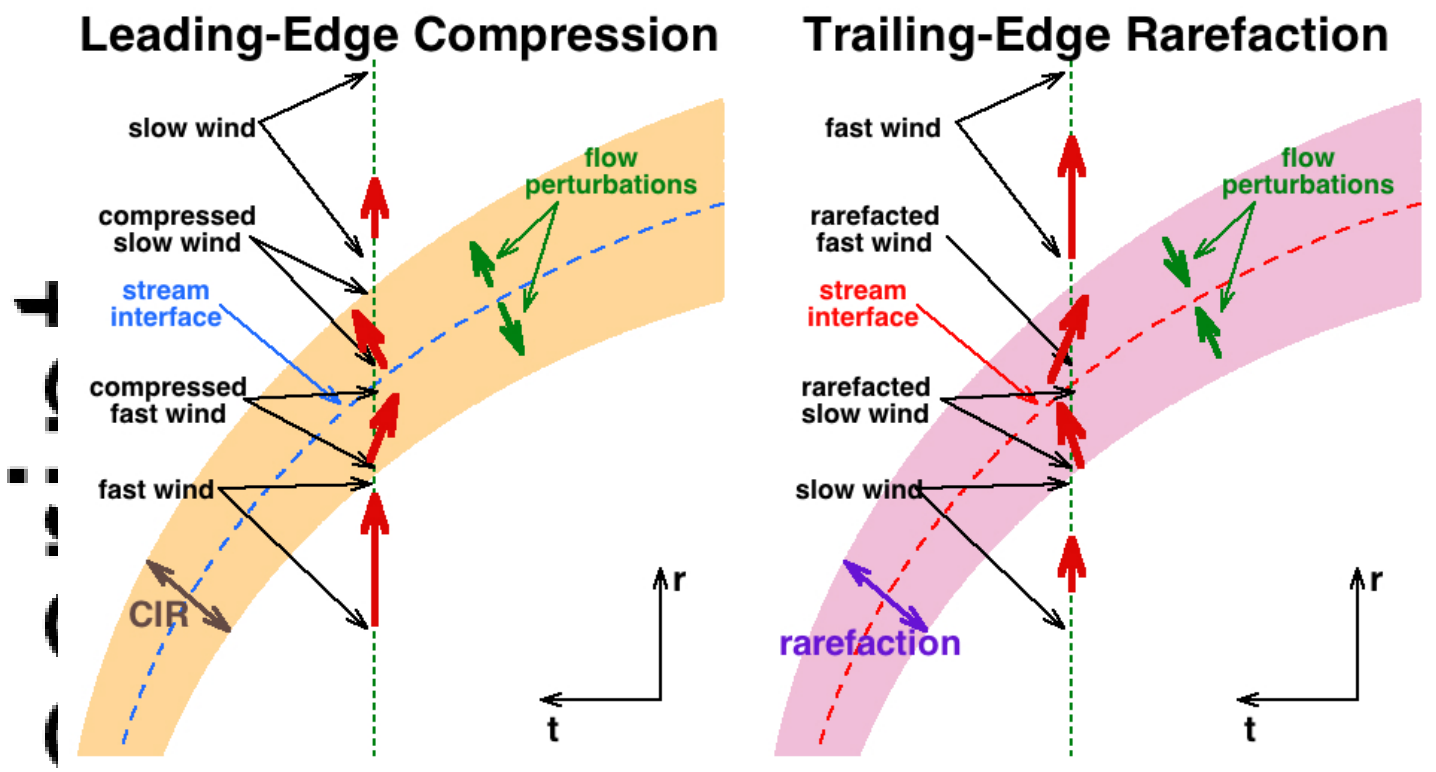

Figure 1 sketch of the compression (CIR) on the leading edge of a high-speed stream (left panel) sketch of the rarefaction on the trailing edge of a high-speed stream (right panel). Both setches are drawn in the ecliptic plane with the RTN coordinate system indicated in the lower right.

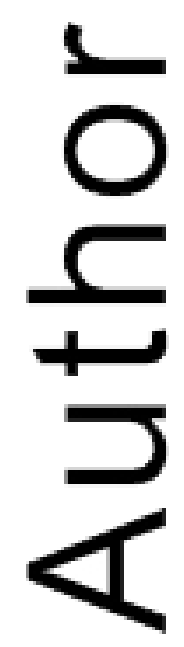

This article is protected by copyright. All rights reserved. 


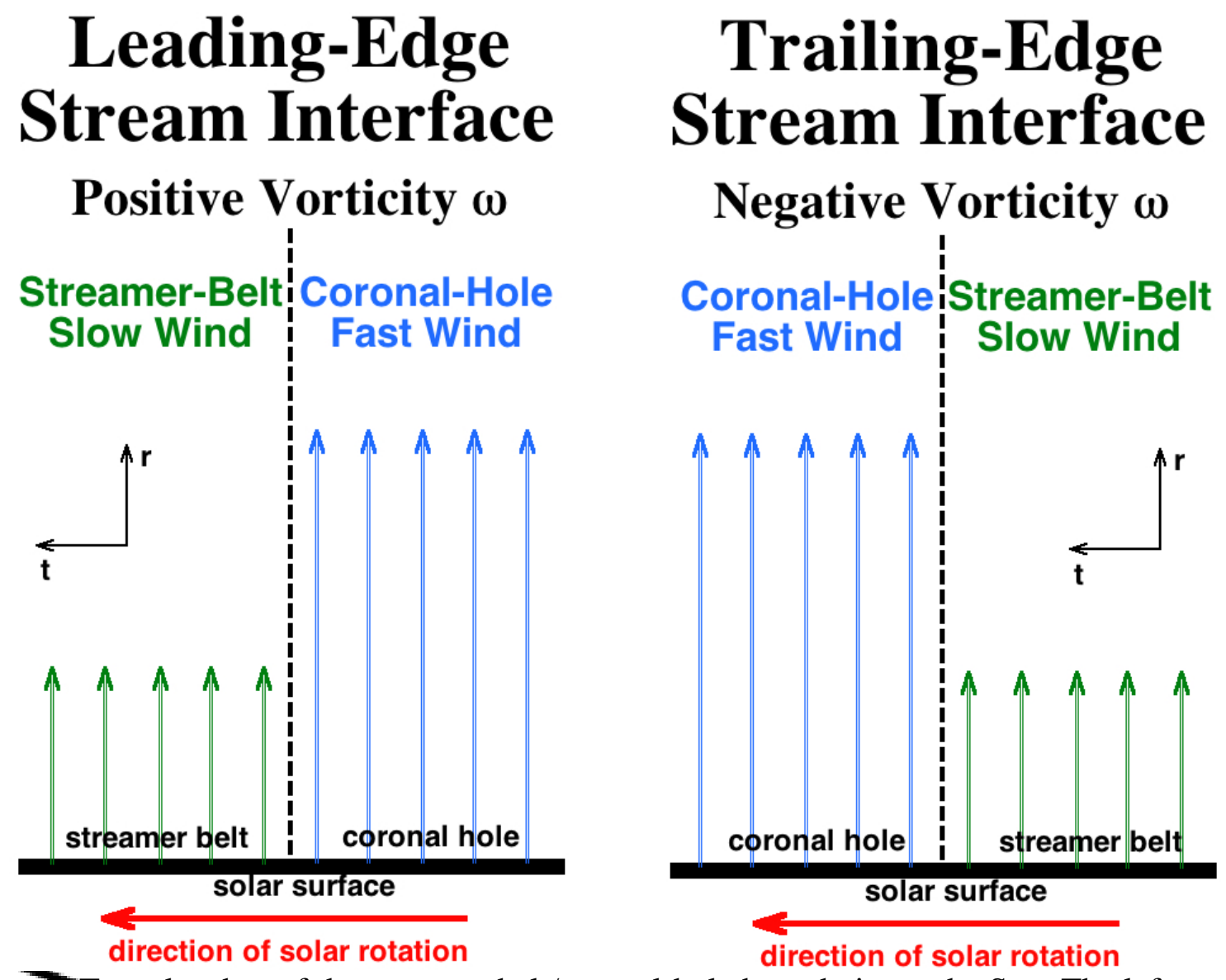

Figure 2. Tyo sketches of the streamer-belt/coronal-hole boundaries at the Sun. The left panel is the b andary at the westward leading edge of a coronal hole and the right panel is the boundary at the eastward trailing edge of a coronal hole. The RTN coordinate system is indicated in the sketches.

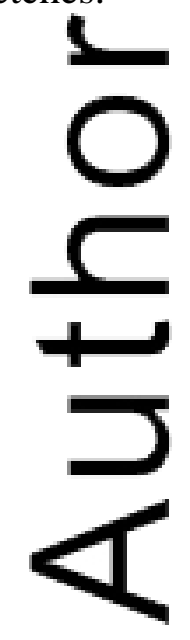



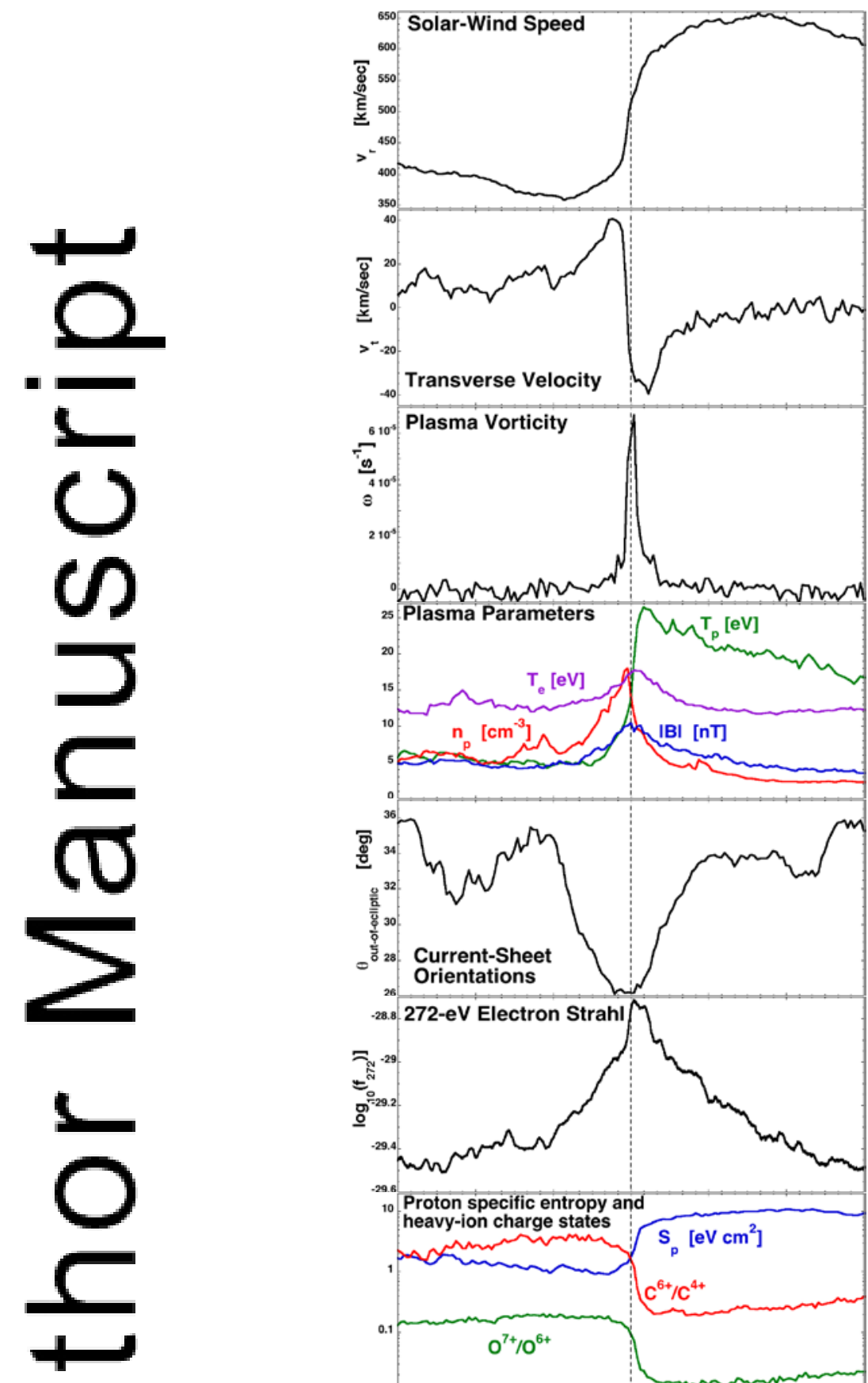

Figure 3 s 3 perposed averages of measurements for the $27 \mathrm{CIR}$ events are plotted. The zero epoch (vertical dasbed line) is the passage of the CIR stream interface as identified with the vorticity peak. Plottedir he top panel is the solar-wind speed (OMNI), in the second panel the transverse velocity of the solar wm (OMNI), the third is the plasma vorticity (ACE SWEPAM), in the fourth panel the plasma 
number density (OMNI), proton temperature (OMNI), magnetic-field strength(OMNI), and electron temperature (Wind 3DP), in the fifth panel the out-of-ecliptic angles of the current-sheet-normal vectors (ACE Mag), in the sixth panel intensity of the 272-eV electron Strahl (ACE SWEPAM), and in the bottom panel the proton specific entropy (OMNI), the carbon charge-state-density ratio (ACE SWICS), and the oxygen charge-state ratio (ACE SWICS).

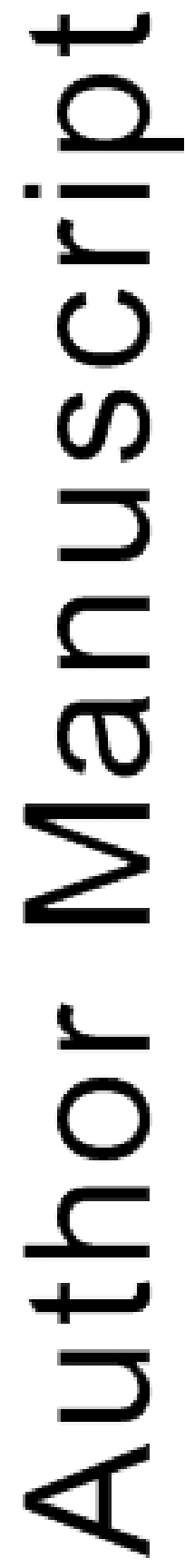



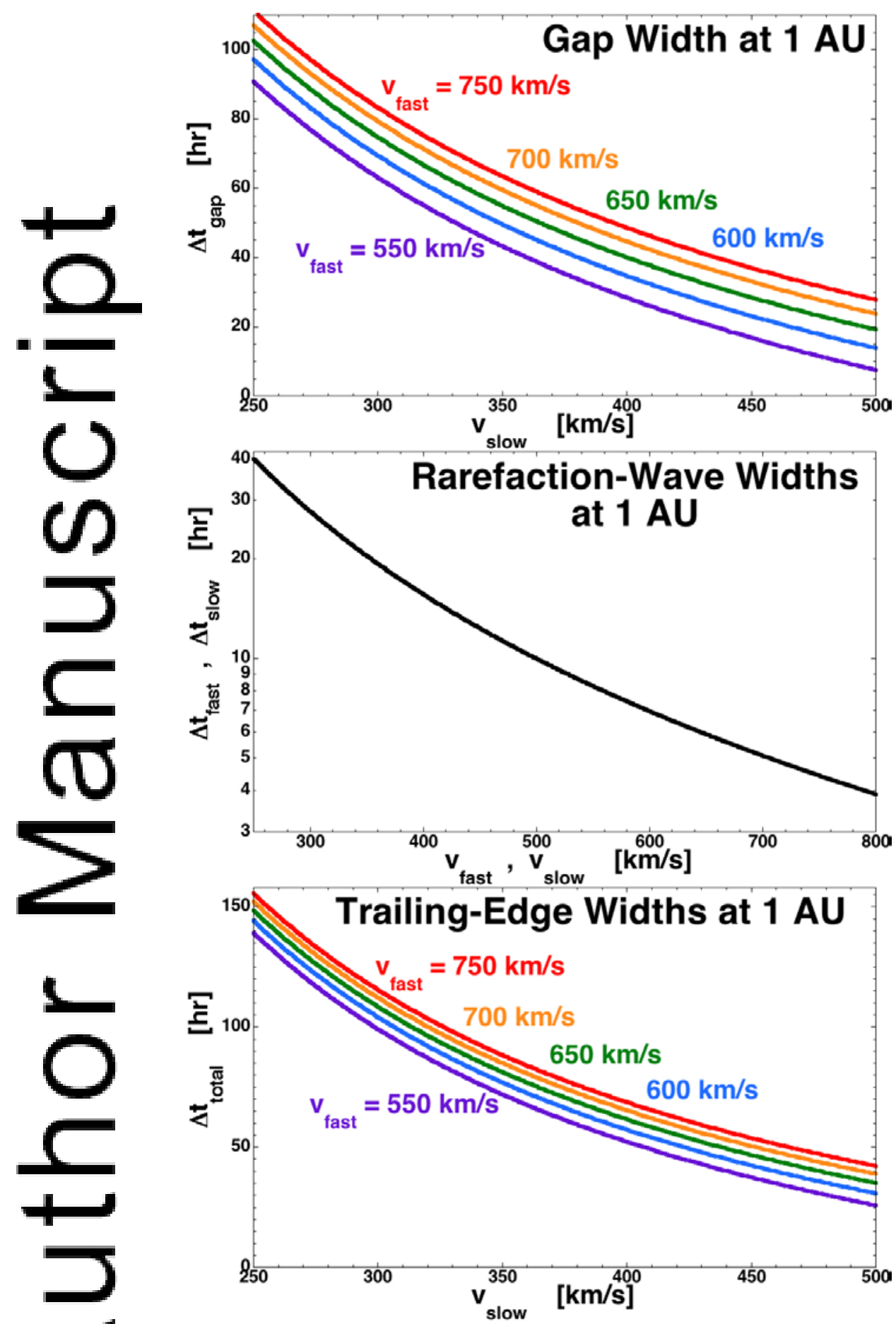

Figure 1 the top panel the estimated temporal widths at $1 \mathrm{AU}$ (from expression (1)) of the balliss ap between the back of a high-speed stream and the front of an interval of slow solar

This article is protected by copyright. All rights reserved. 
wind are plotted. In the middle panel the estimated temporal widths at 1 AU (from expressions (2)) of a pressure driven rarefaction into either the back of a high-speed stream or the front of an interval of slow wind are plotted. In the bottom panel the total temporal width of a rarefaction perturbation at $1 \mathrm{AU}$ as given by expression (3) is plotted.

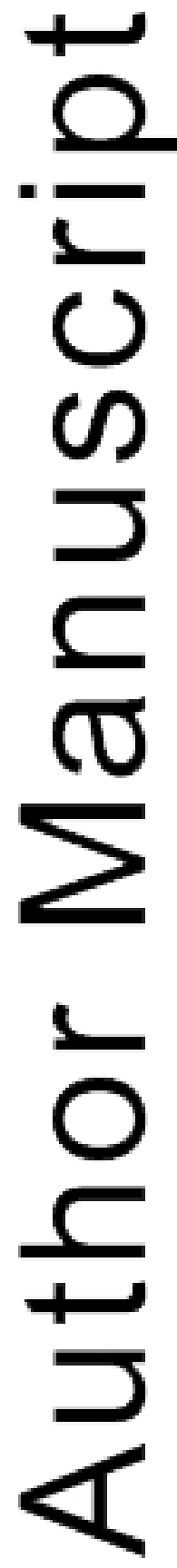




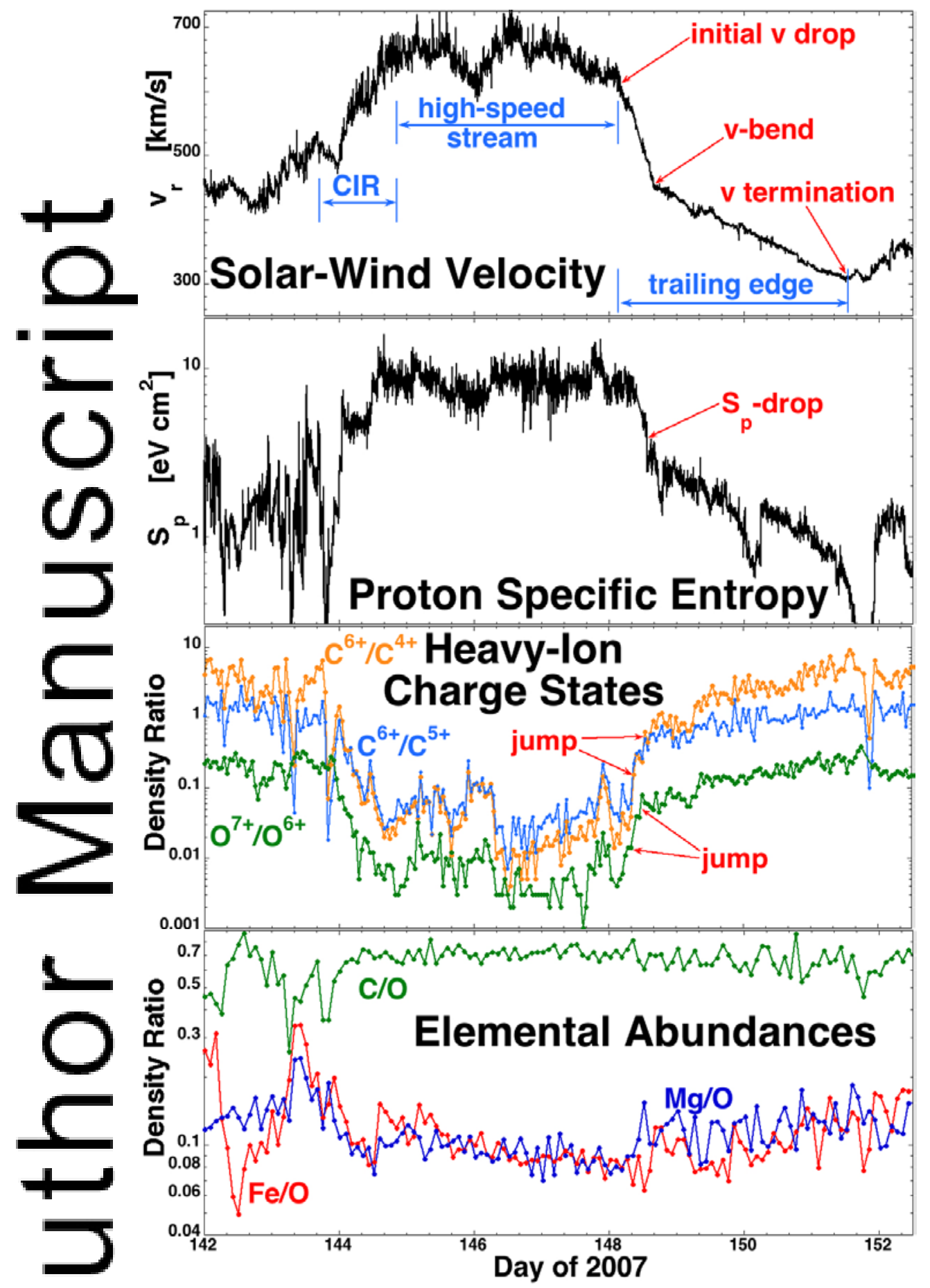

Figure 5er a time period of 10.5 days several relevant solar-wind parameters are plotted for Trailno dge \# 45 of Table 1 and for the high-speed stream that precedes the trailing edge. The

This article is protected by copyright. All rights reserved. 
top two panels are measurements from Wind SWE and the bottom two panels are measurements from Ace SWICS.

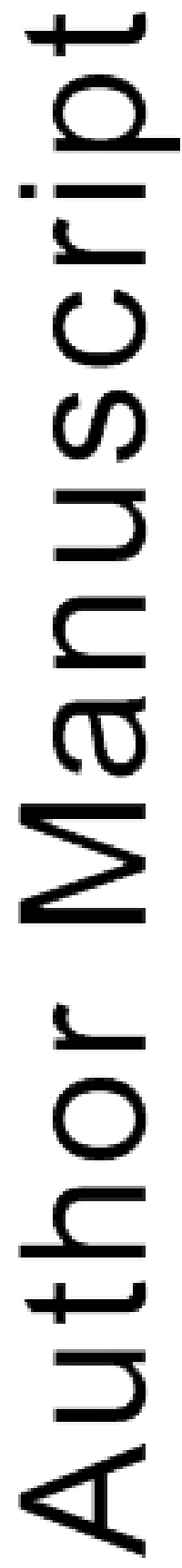




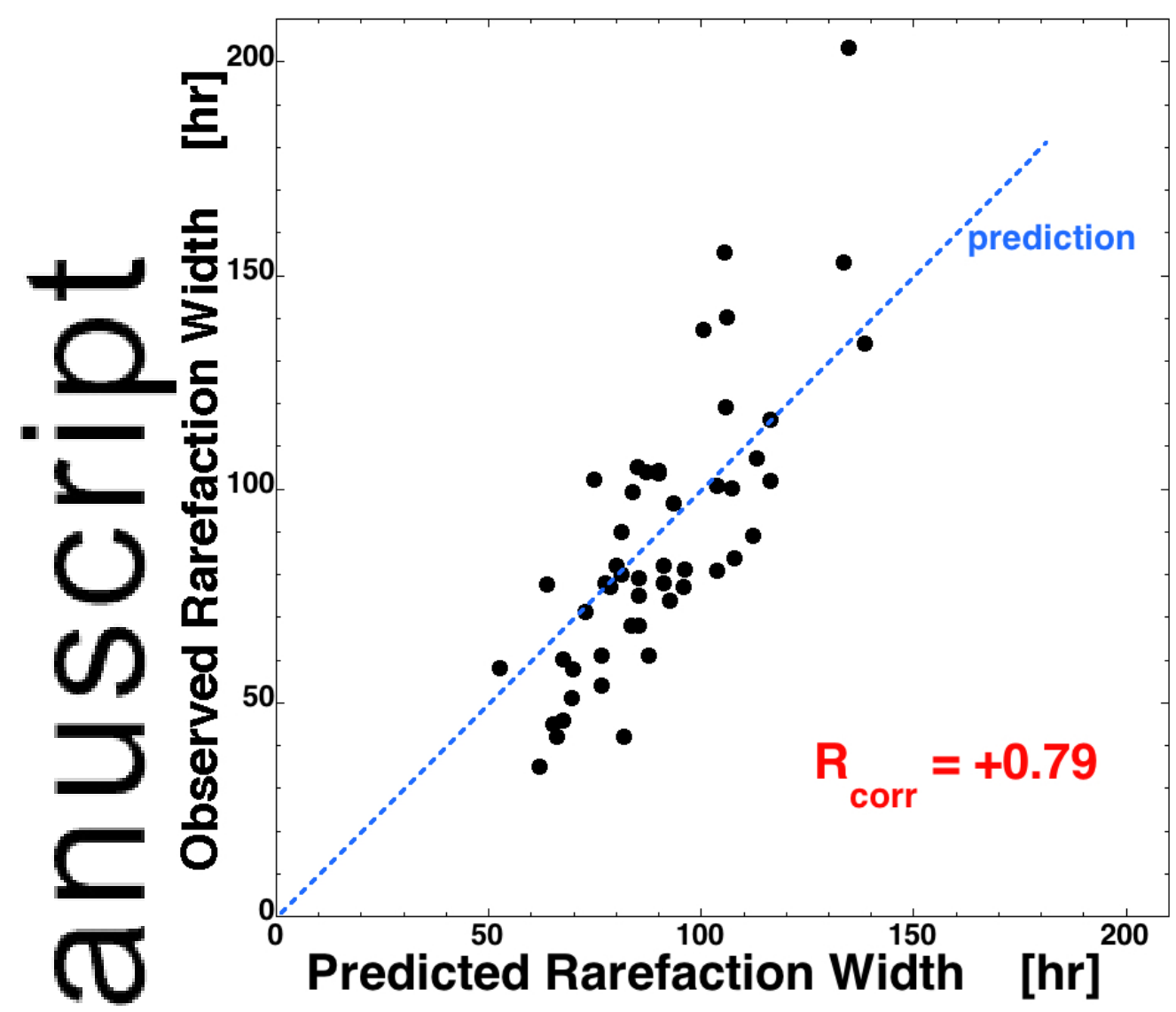

Figure 6 Eor the trailing edges listed in Table 1, the observed temporal width at 1 AU of each trailing idge is plotted vertically versus the predicted trailing edge width from expression (3) using measured velocities of the slow and fast wind bounding each trailing edge. The dashed blue curve (not a fit) indicates where observation would match prediction.

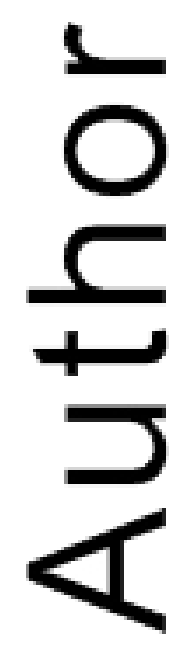



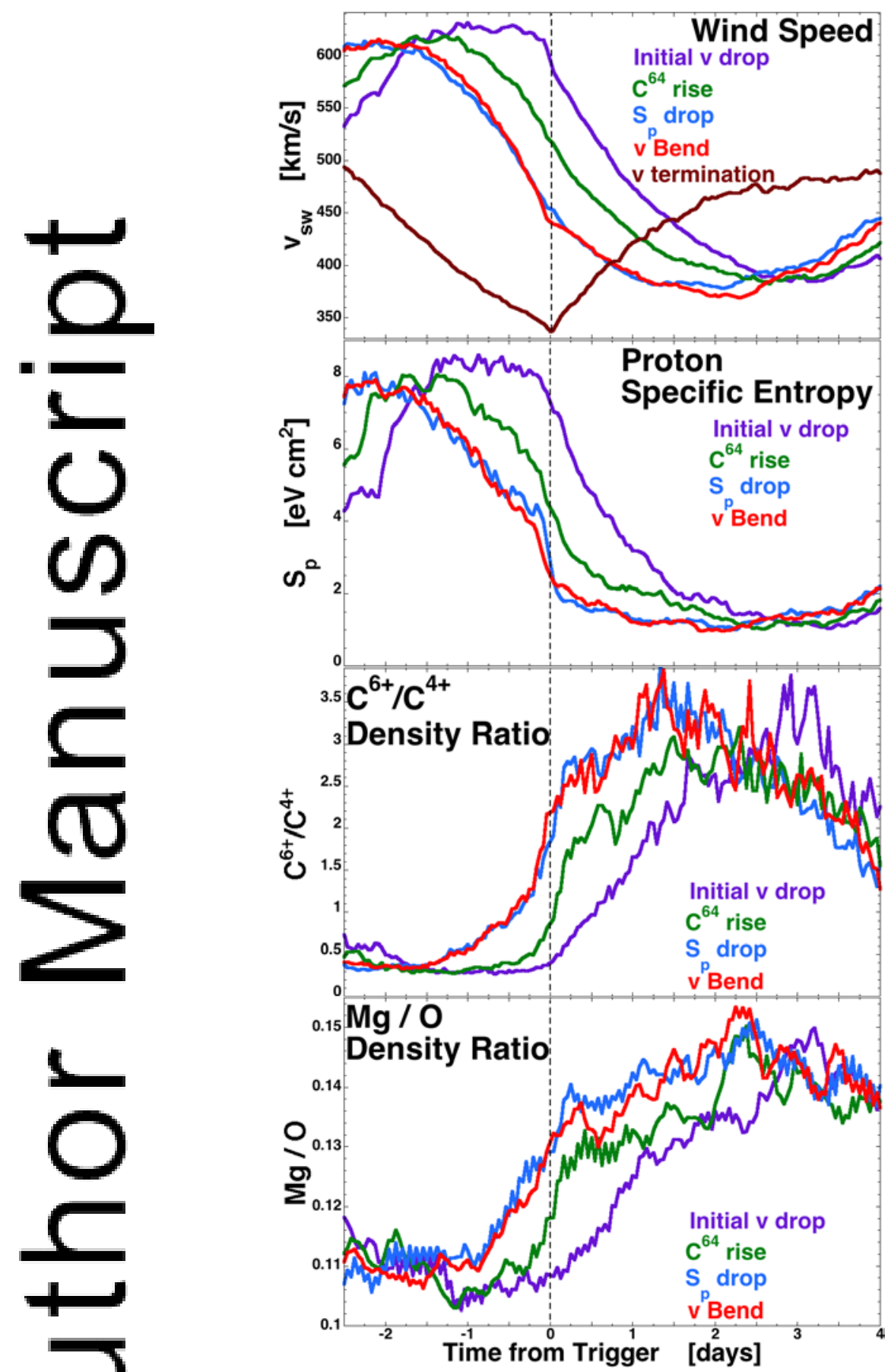

Figure superposed averages of candidate signatures for trailing-edge stream-interface identifiers plotted vertically as functions of time with time organized according to various trigg . The top panel plots vsw (OMNI), the second panel plots the proton specific entropy

This article is protected by copyright. All rights reserved. 
(OMNI), the third panel a carbon charge-state-density ratio ACE SWICS), and in the bottom panel plots the magnesium-to-oxygen elemental abundance ratio (ACE SWICS). The four colored curves are superposed averages triggered on the initial velocity drop (purple), the rise in $\mathrm{C}^{6+} / \mathrm{C}^{4+}$ (green), the drop in $\mathrm{S}_{\mathrm{p}}$ (blue), and the velocity-bend (red). In the top panel and extra curve (brown) is triggered on the velocity terminations of the trailing edge refractions.

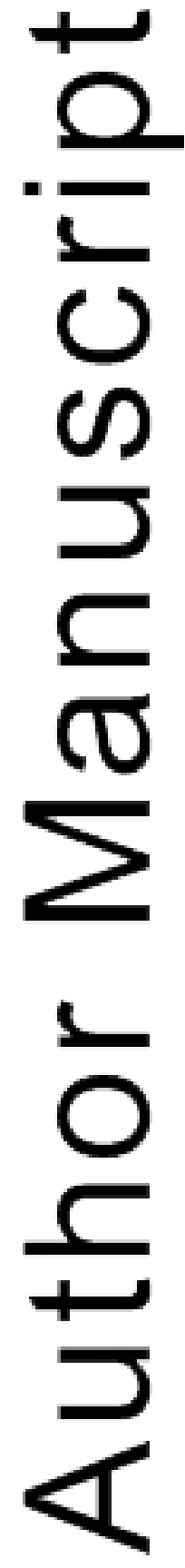




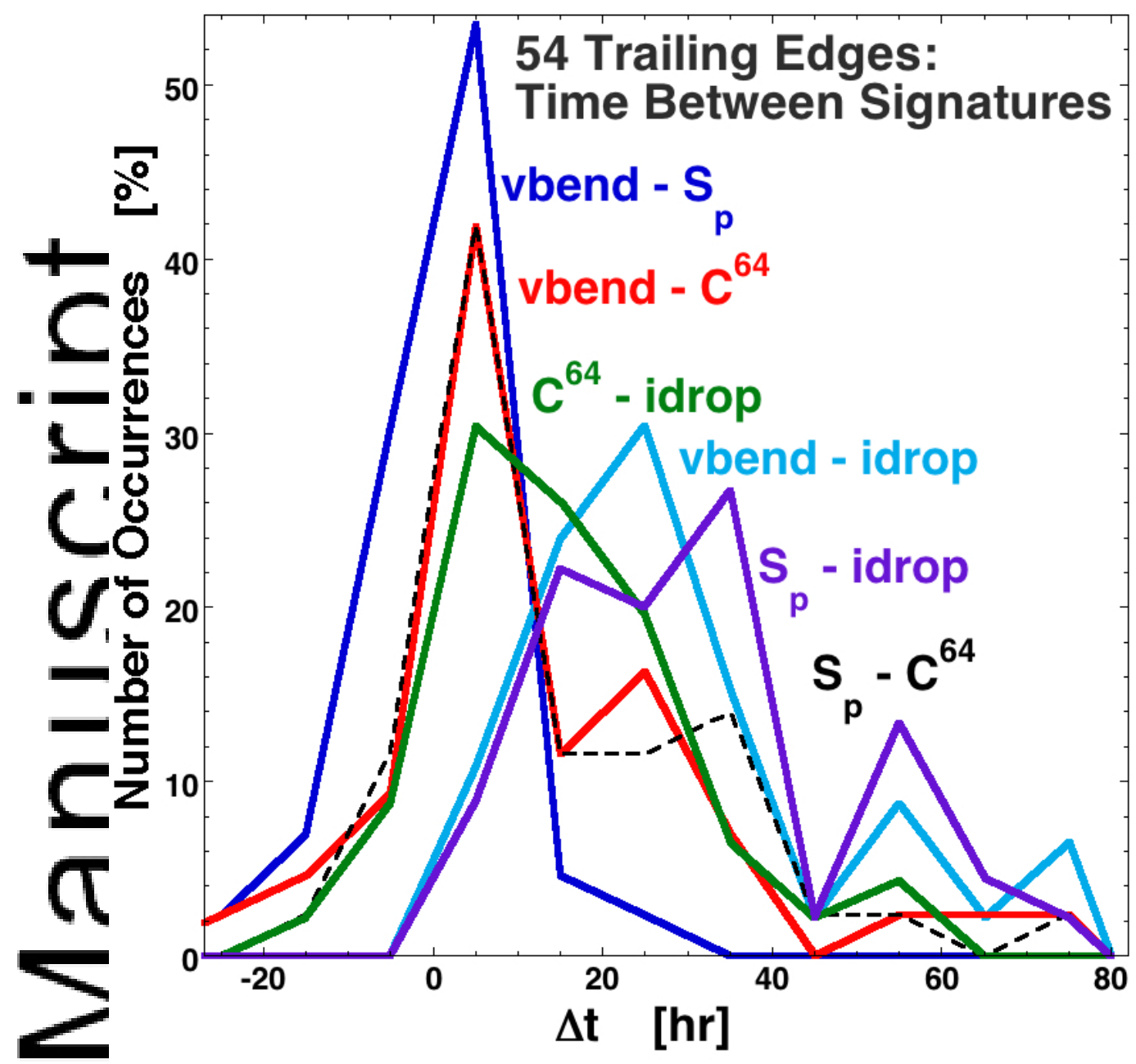

Figure 8. For the 54 trailing edges listed in Table 1, times are recorded for the initial velocity drop, the velocity-bend, the rise in $\mathrm{C}^{6+} / \mathrm{C}^{4+}$, and the drop in $\mathrm{S}_{\mathrm{p}}$. In the figure the time differences betweentnese various time signatures are binned for the 54 trailing edges.

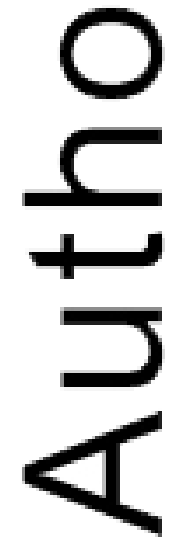



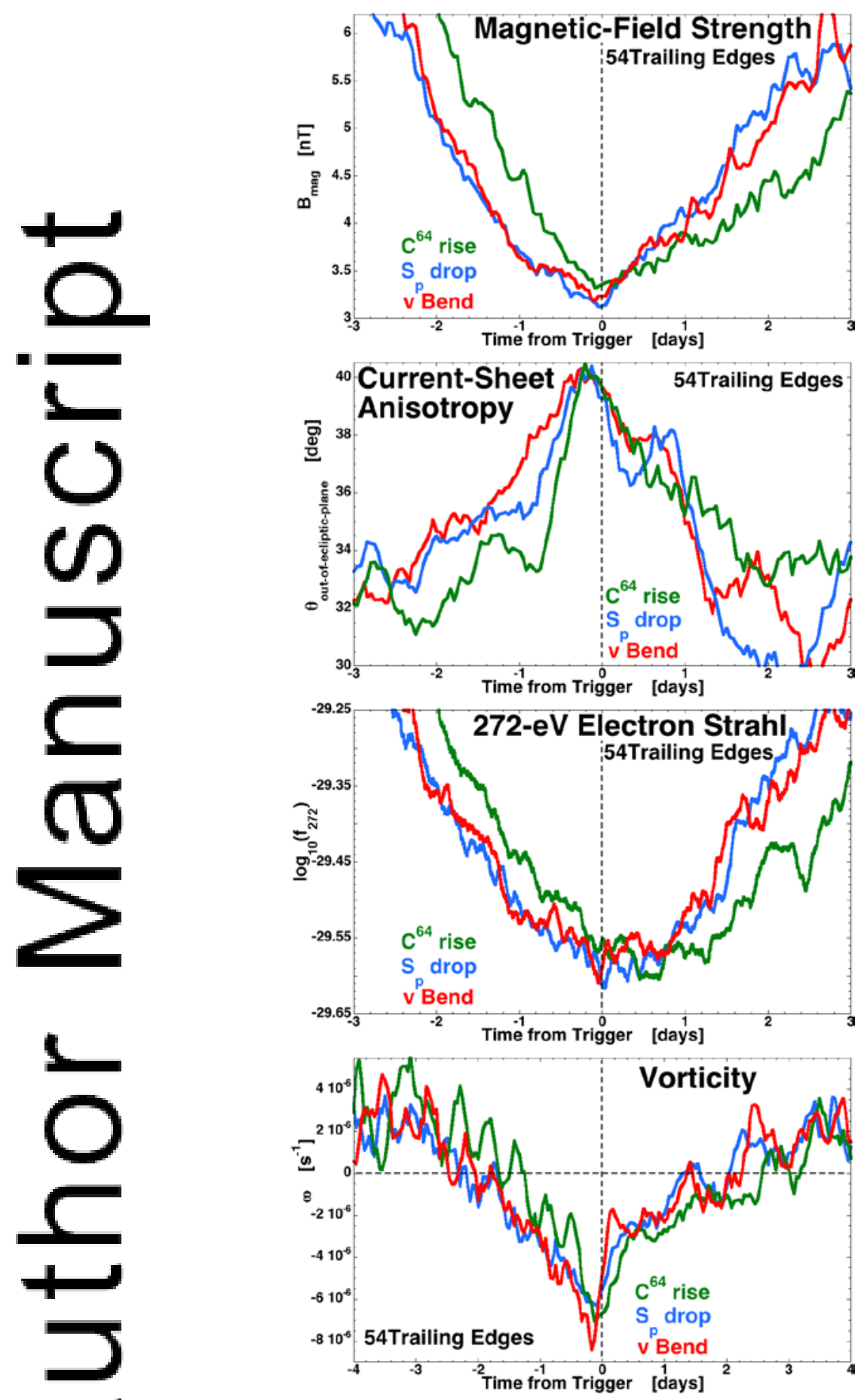

Figure 9-aperposed averages of four diagnostic parameters for the trailing-edge refractions are plottar functions of time. In the top panel the magnetic field strength (OMNI), in the second

This article is protected by copyright. All rights reserved. 
panel the out-of-ecliptic angle of the current sheets (ACE Mag), in the third panel the intensity of the $272 \mathrm{eV}$ electron scrawl (ACE SWEPAM), and in the bottom panel the plasma vorticity (ACE SWEPAM). In each panel the green curve is a superposed epoch average triggered on the $\mathrm{C}^{6+} / \mathrm{C}^{4+}$ rise, the blue curve an average triggered on the $\mathrm{S}_{\mathrm{p}}$ drop, and the red curve an average triggered on the velocity-bend.

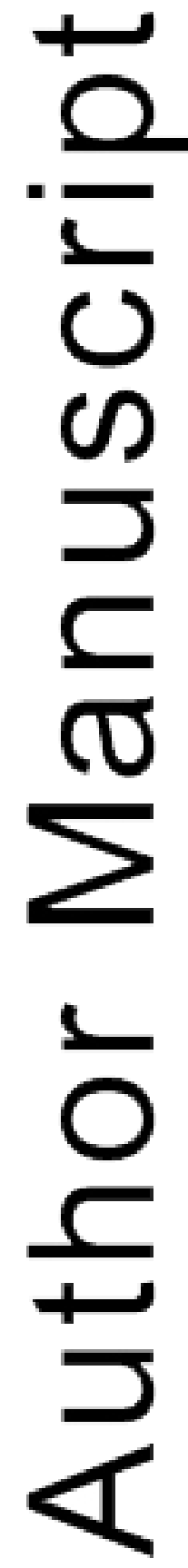




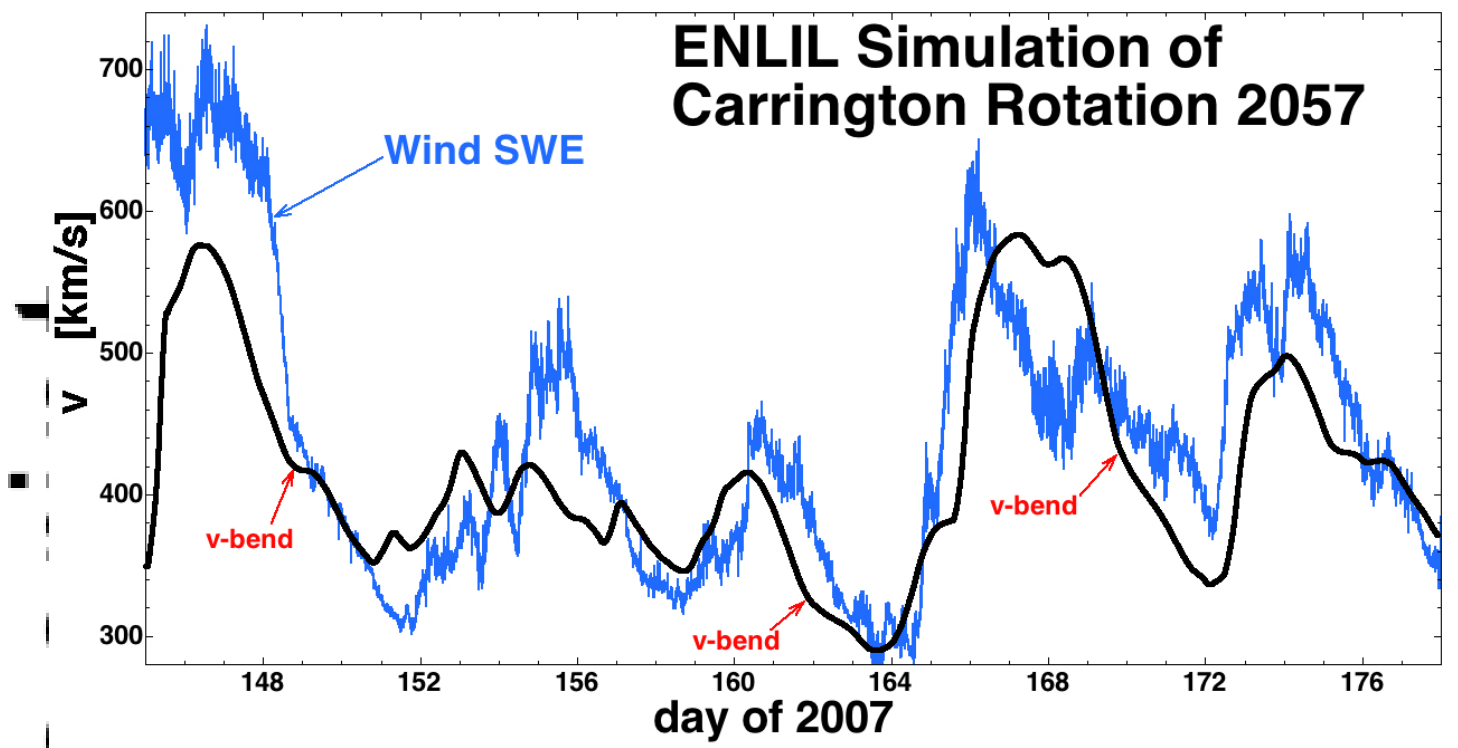

Figure T0. For an ENLIL 3-D MHD computer simulation of Carrington Rotation 2057 catalogueart the CCMC (run Lan_Jian_121311_SH_3), the solar-wind radial velocity at Earth is plotted-lack. Also plotted in blue is the solar-wind speed measured by Wind SWE. The velocity hands seen on the trailing edges in the ENLIL simulation are marked in red.

তে

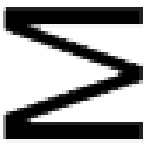

3

O

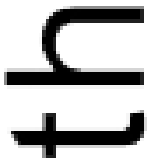

定

This article is protected by copyright. All rights reserved. 


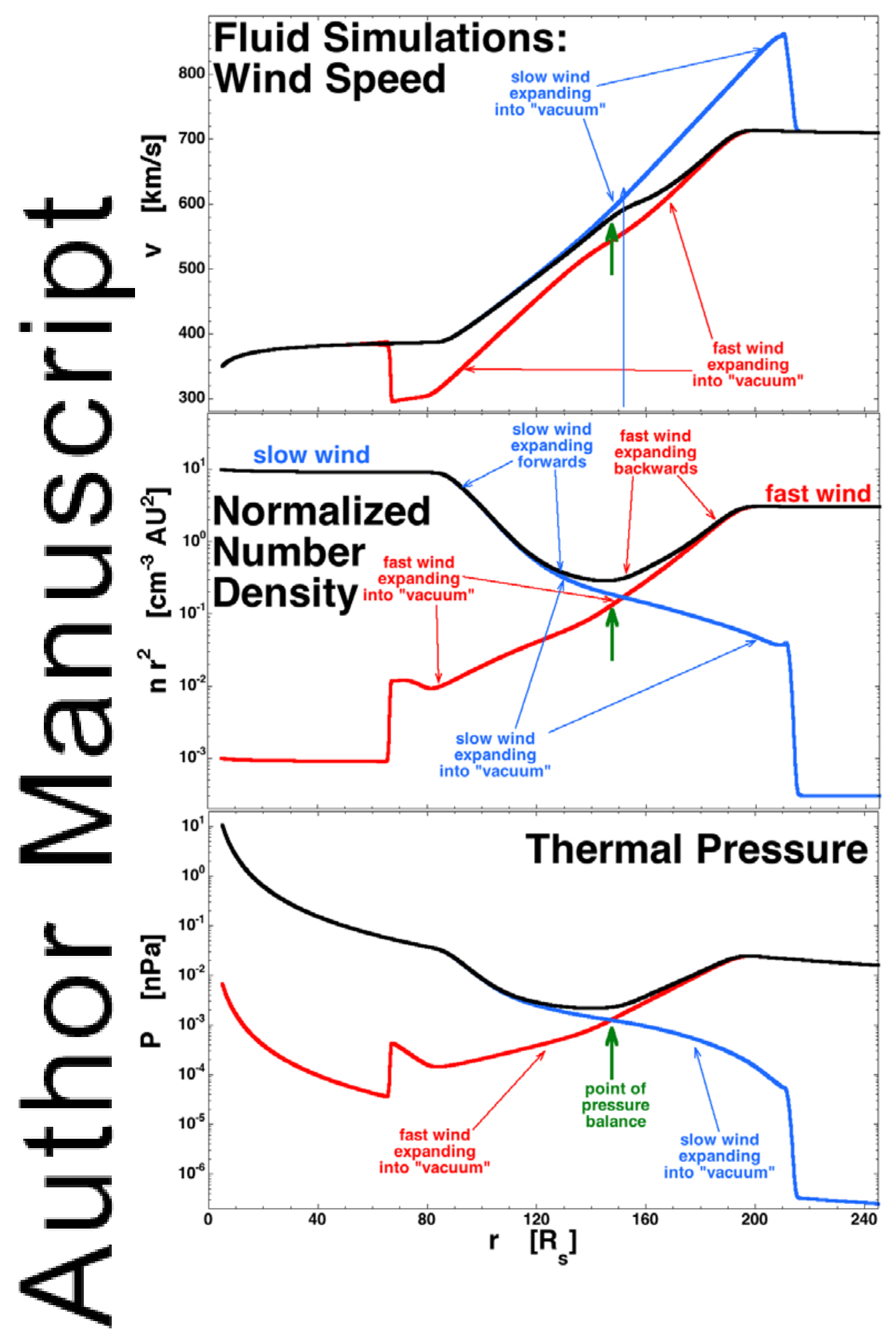

This article is protected by copyright. All rights reserved. 
Figure 11. Using a 1-dimensional (radial) fluid-simulation code in spherical geometry, three simulations are run and various quantities are plotted as a function of radius at the same time in each of the three simulations. The black curves are from a simulation with $n_{\text {fast }}=3 \mathrm{~cm}^{-3}$ and $n_{\text {slow }}$ $=10 \mathrm{~cm}^{-3}$ (at 1AU); the red curves are from a simulation with $\mathrm{n}_{\text {fast }}=3 \mathrm{~cm}^{-3}$ and $\mathrm{n}_{\text {slow }}=0.001 \mathrm{~cm}^{-}$ ${ }^{3}$; the blue curves are from a simulation with $\mathrm{n}_{\text {fast }}=0.0003 \mathrm{~cm}^{-3}$ and $\mathrm{n}_{\text {slow }}=10 \mathrm{~cm}^{-3}$.

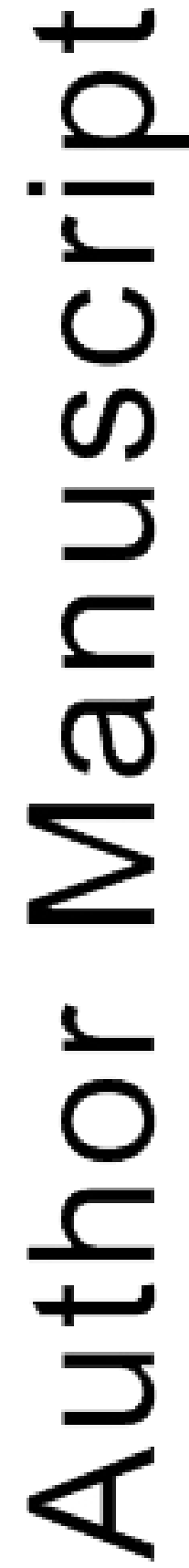




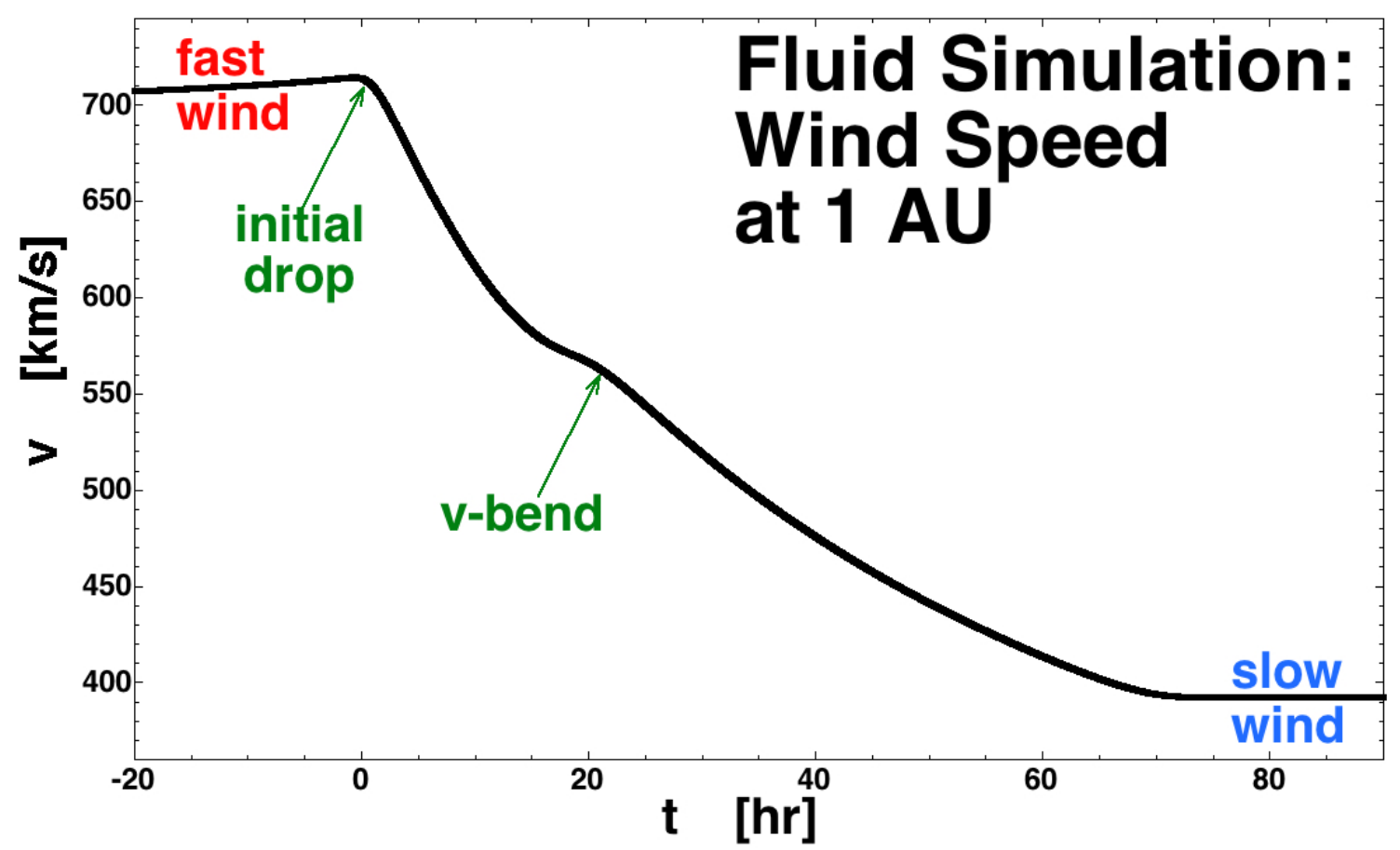

Figure 2. From the black-curve simulation of Figure 11, the solar-wind speed at 1AU is plotted as a fumen of time. Time $\mathrm{t}=0$ is arbitrarily taken as the time when the initial velocity drop occurs
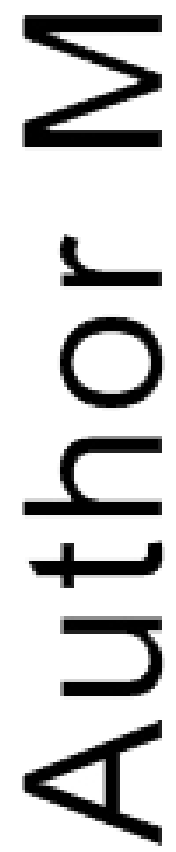

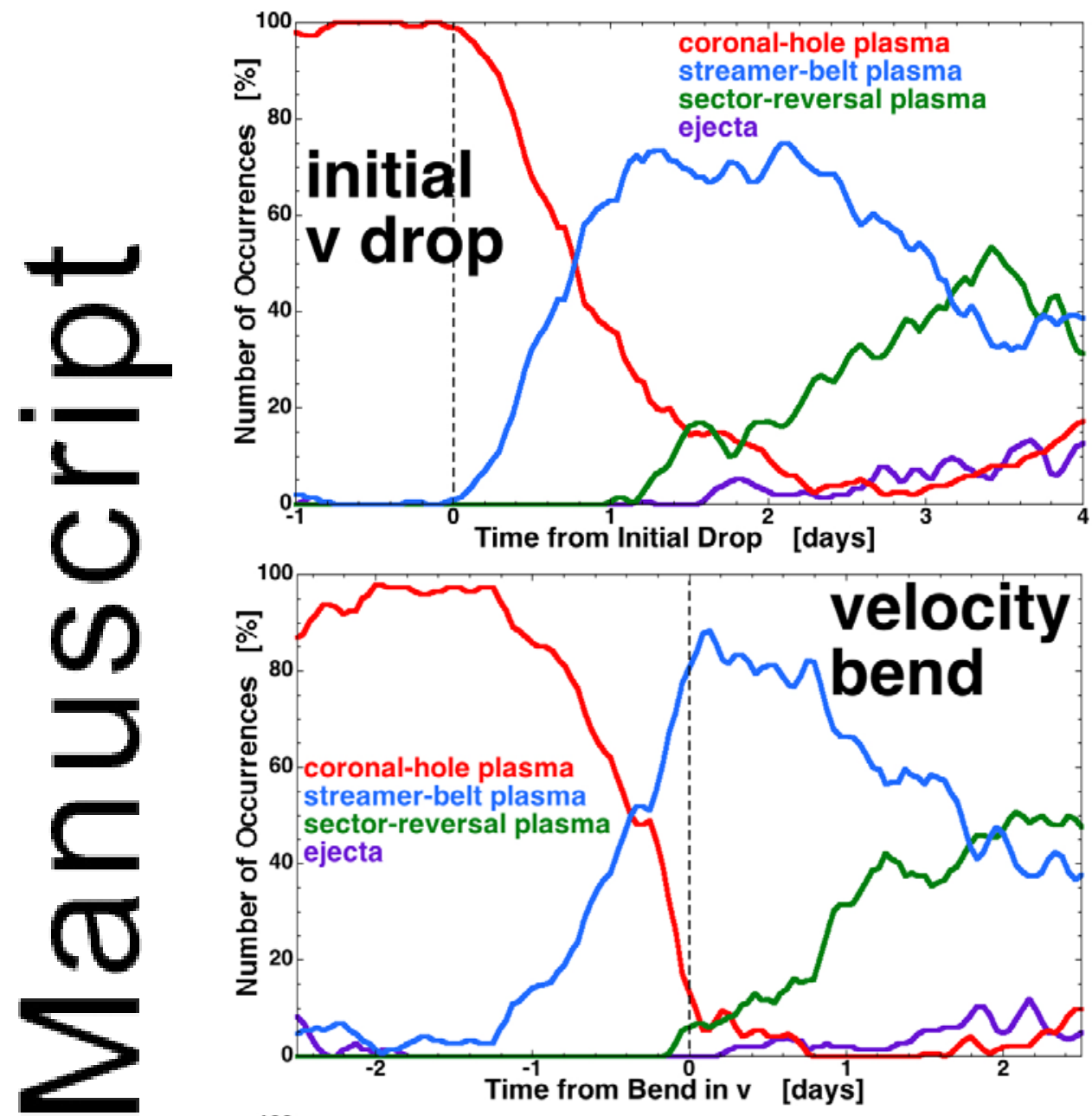

Thend in $\mathbf{v}$ [days]
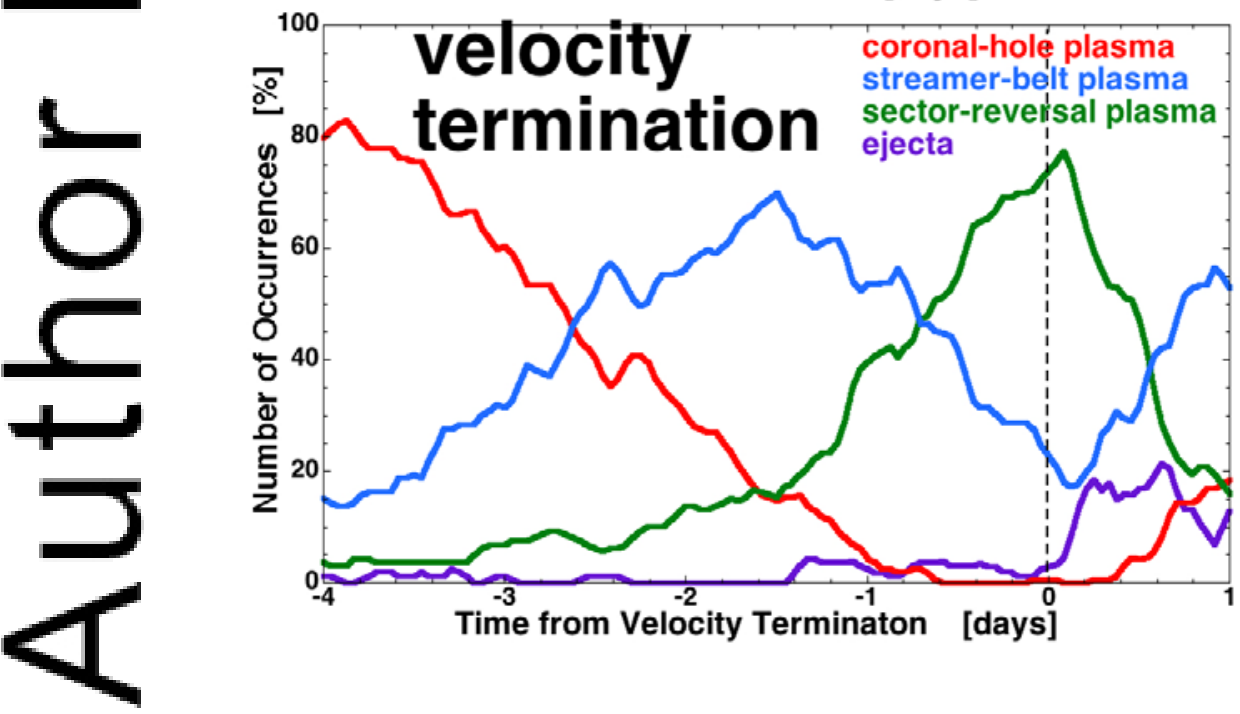

This article is protected by copyright. All rights reserved. 
Figure 13. Using the $X u$ and Borovsky [2015] algebraic scheme to categorize the type of solar wind plasma at $1 \mathrm{AU}$ for the 54 trailing edges in Table 1, the percentage of time that each of the four types of plasmas appears is plotted as a function of time in four colors. In the top panel time is organized about the initial velocity drop $(\mathrm{t}=0)$ of the 54 trailing edges, in the middle panel time is organized about the velocity-bend $(\mathrm{t}=0)$ of the 54 trailing edges, and in the bottom panel time is organized,about the velocity termination $(t=0)$ of the 54 trailing edges.

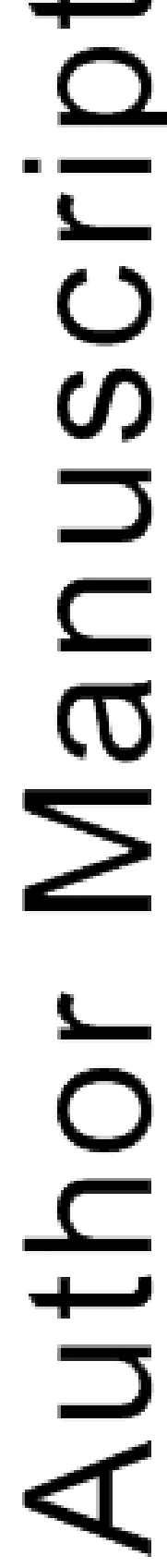



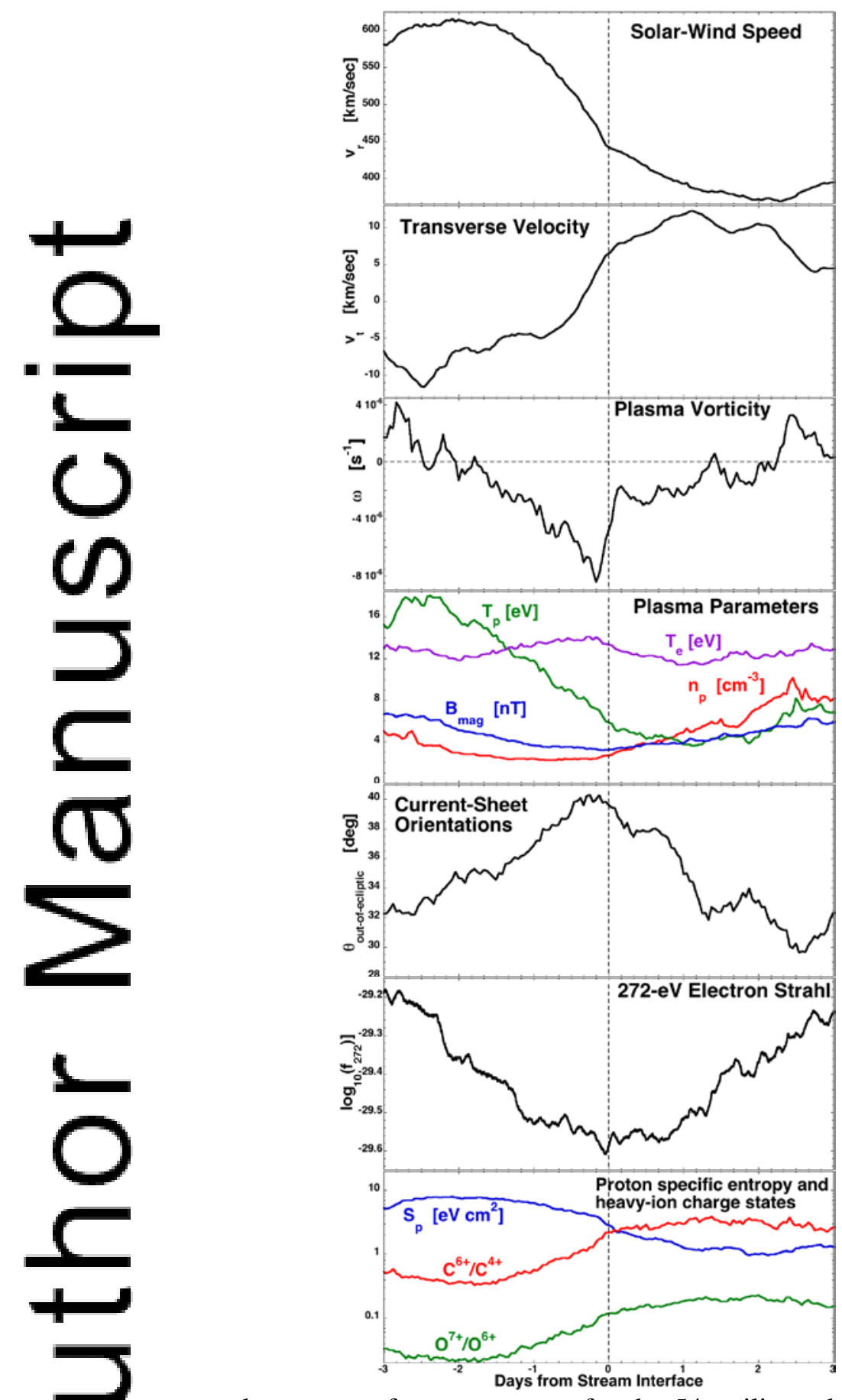

Figure 14. Superposed averages of measurements for the 54 trailing edges of Table 1 are plotted. The zero epon (vertical dashed line) is the passage of the velocity bend in each trailing edge. Plotted in the top ponel the solar-wind speed (OMNI), in the second panel the transverse velocity of the solar wind 
(OMNI), the third is the plasma vorticity (ACE SWEPAM), in the fourth panel the plasma number density $(\mathrm{OMNI})$, proton temperature (OMNI), magnetic-field strength (OMNI), and electron temperature (Wind 3DP), in the fifth panel the out-of-ecliptic angles of the current-sheet-normal vectors (ACE Mag), in the sixth panel intensity of the 272-eV electron strahl (ACE SWEPAM), and in the bottom panel the proton specific entropy (OMNI), the carbon charge-state-density ratio (ACE SWICS), and the oxygen charge-state ratio (ACE SWICS).

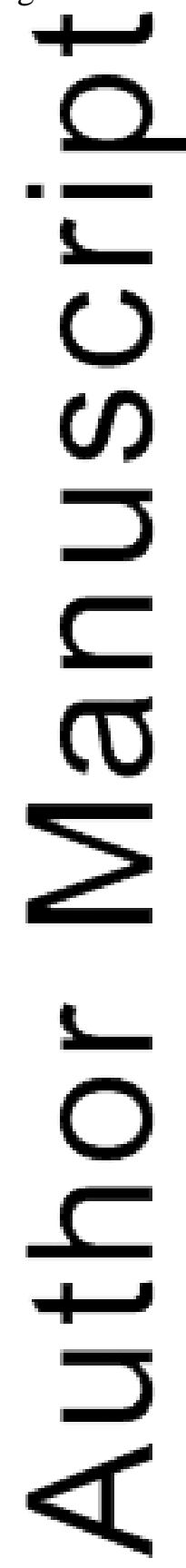




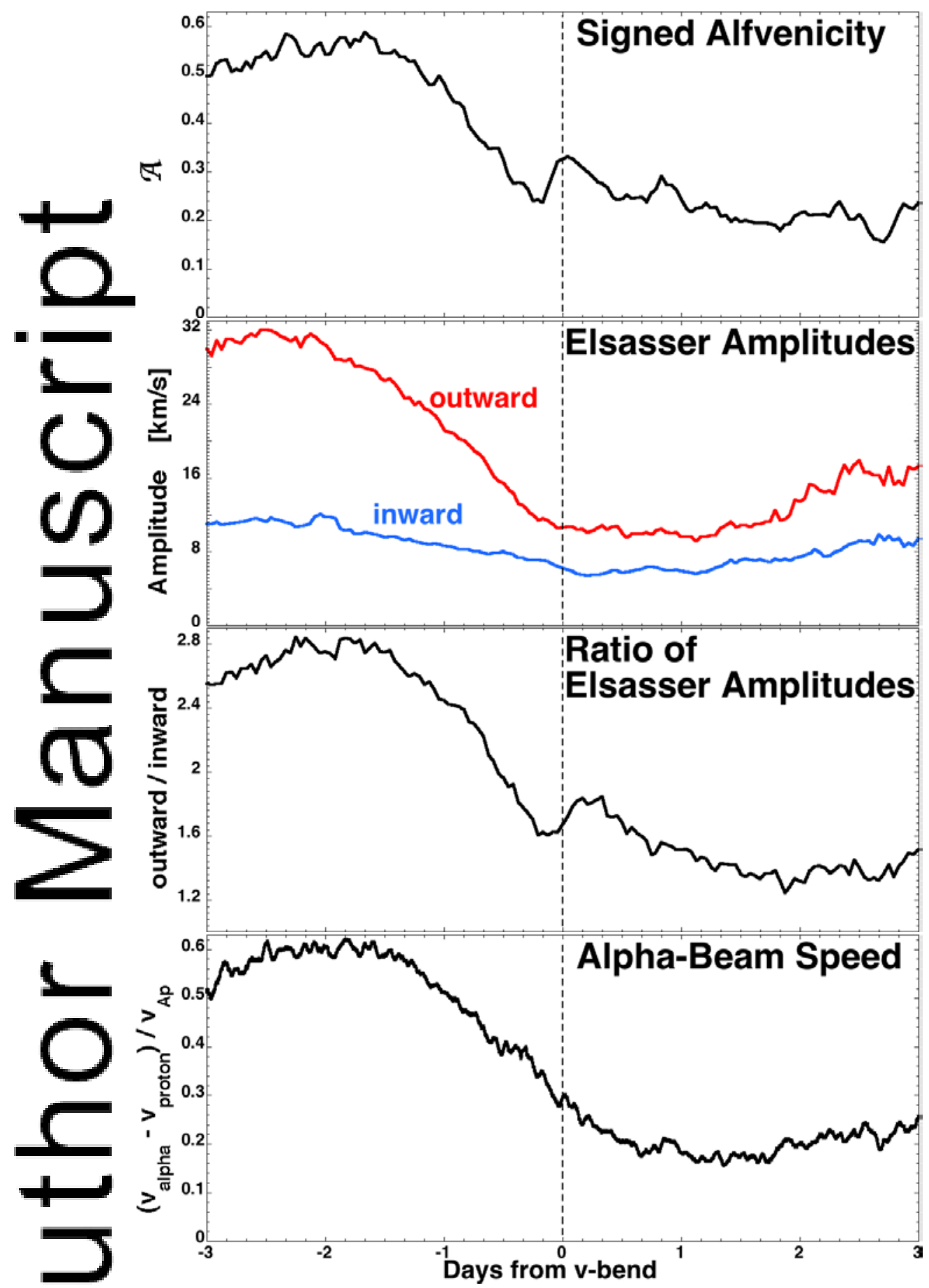

Figure 15. Triggered on the velocity-bend of the 54 trailing edges in Table 1, the top panel plots the seer osed average of the signed Alfvenicity (expression (5)) (ACE SWEPAM and MAG),

This article is protected by copyright. All rights reserved. 
the second panel plots the superposed averages of the amplitudes of the outward-sense (red) and inward-sense (blue) Elsasser fluctuations (ACE SWEPAM and MAG), the third panel plots the superposed average of the outward-to-inward Elsasser amplitude, and the bottom panel plots the superposed average of the velocity of the alpha beam relative to the proton velocity (normalized to the proton Alfven speed) (WIND SWE and Mag).

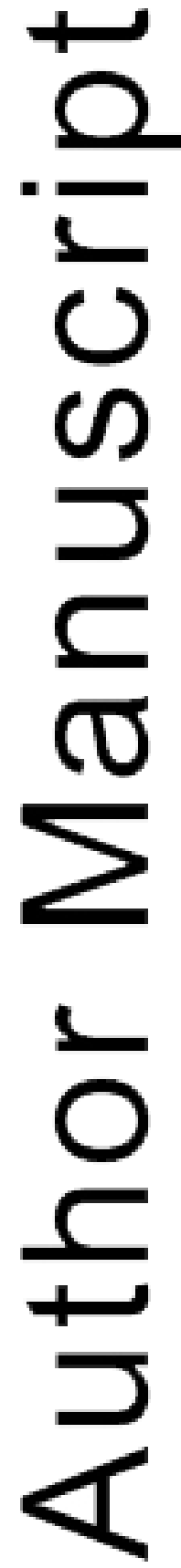



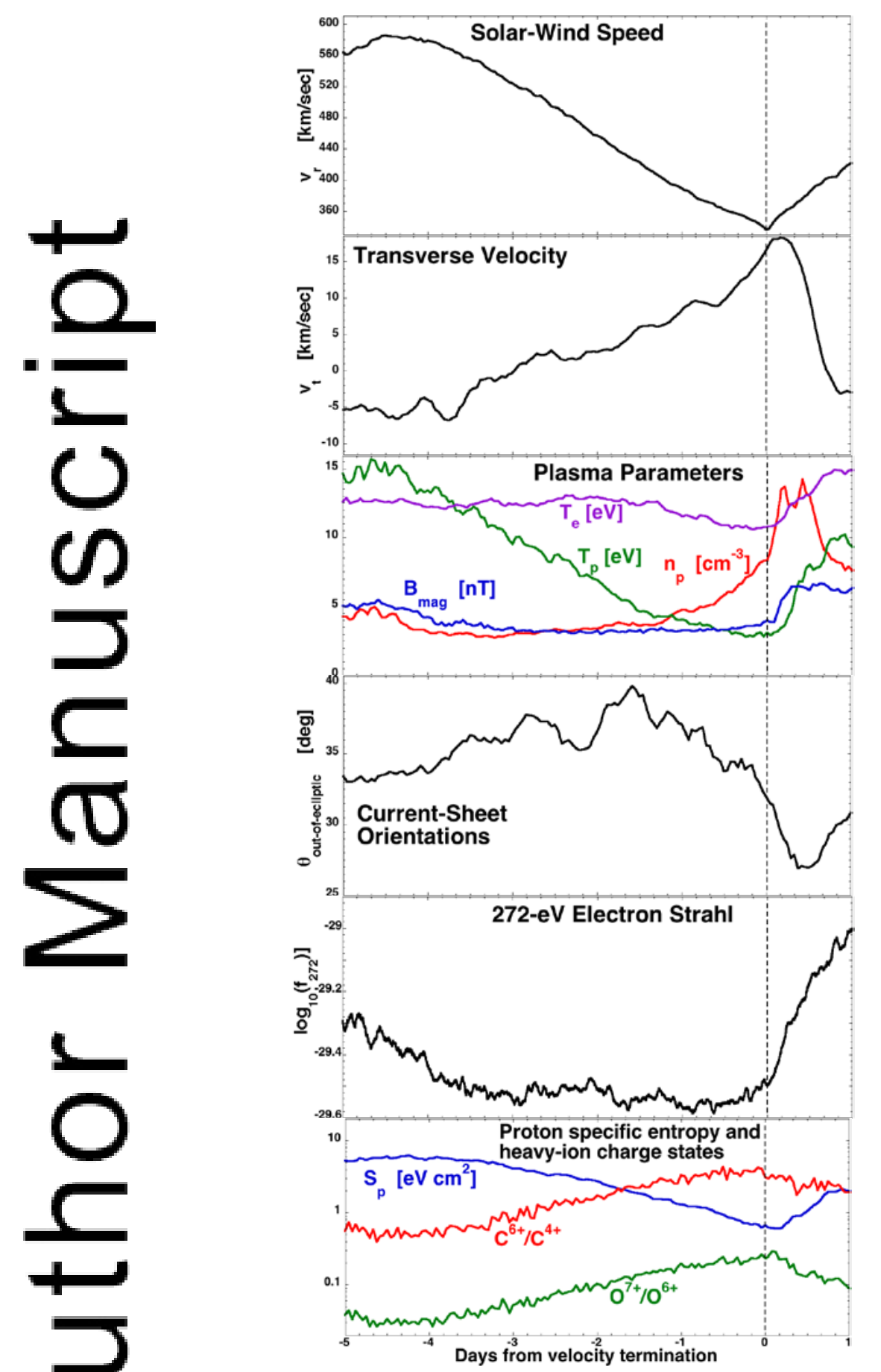

Figure 16. Superposed averages of measurements for the 54 trailing edges of Table 1 are plotted. The zero epen (vertical dashed line) is the passage of the velocity termination in each trailing edge. Plotted in the topana hel is the solar-wind speed (OMNI), in the second panel the transverse velocity of the solar 
wind (OMNI), in the third panel the plasma number density (OMNI), proton temperature (OMNI), magnetic-field strength (OMNI), and electron temperature (Wind 3DP), in the fourth panel the out-ofecliptic angles of the current-sheet-normal vectors (ACE MAG), in the fifth panel intensity of the 272-eV electron strahl (ACE SWEPAM), and in the bottom panel the proton specific entropy (OMNI), the carbon charge-state-density ratio (ACE SWICS), and the oxygen charge-state ratio (ACE SWICS).

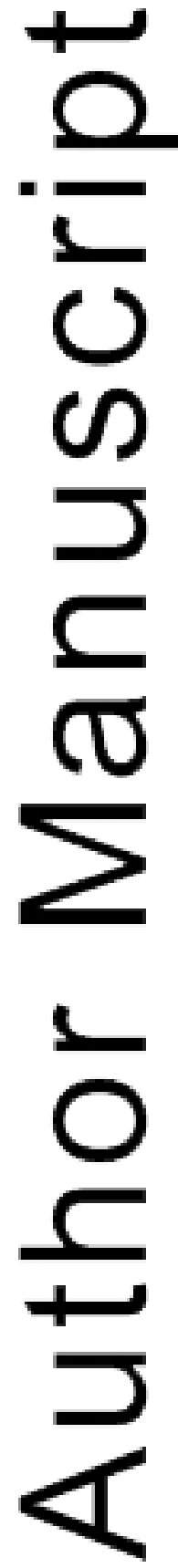




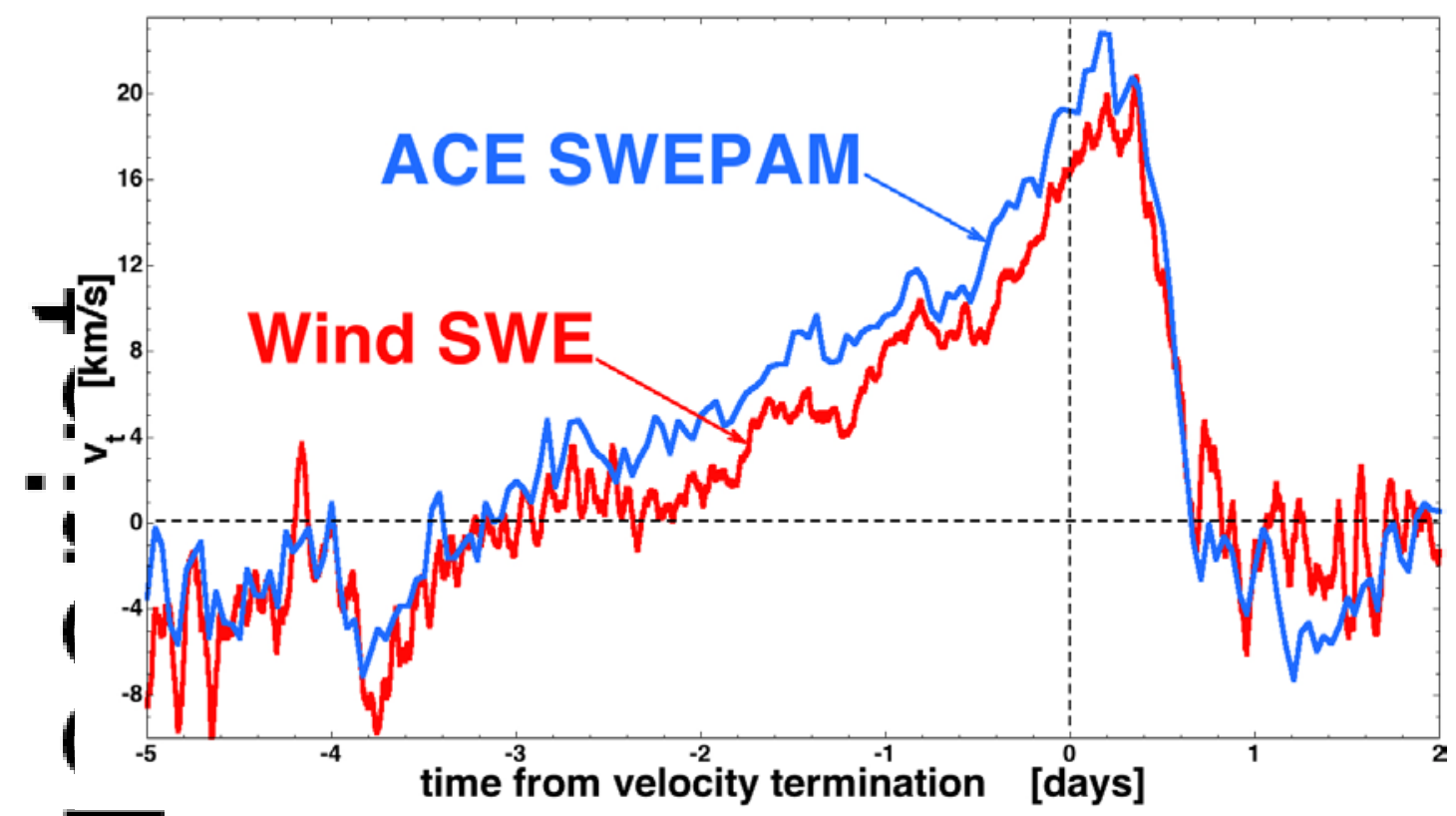

Figure T7. So check the veracity of the transverse velocity being maximum at the termination of the vel decline, superposed averages are plotted separately for ACE electrostatic-analyzer measuremants and Wind Faraday-cup measurements, both triggered on the velocity terminations of the 4 trailing edges in Table 1 .

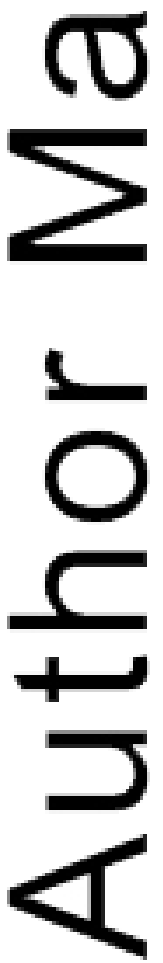

This article is protected by copyright. All rights reserved. 


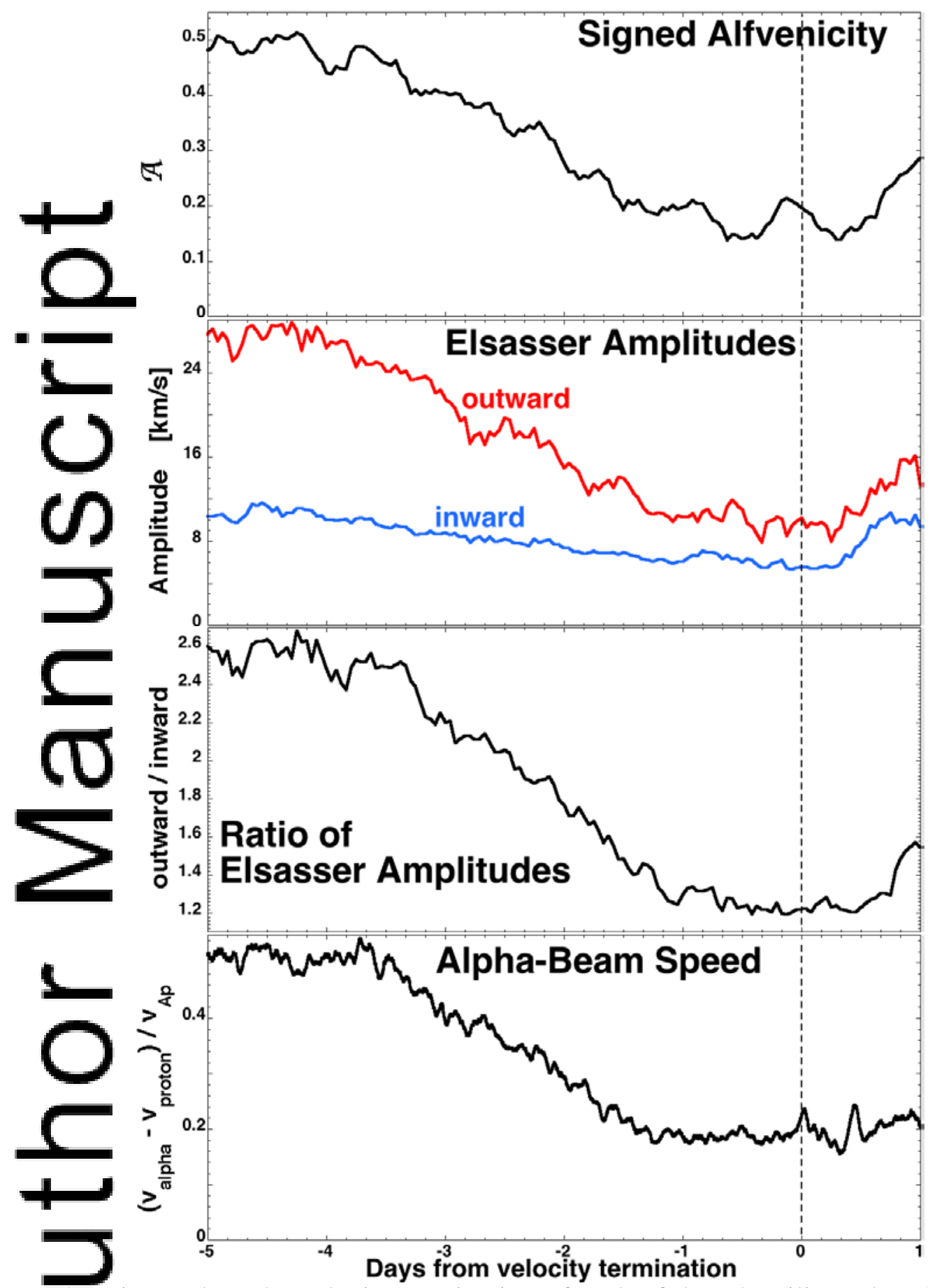

Figure 18. Triggered on the velocity termination of each of the 54 trailing edges in Table 1, the top pare plots the superposed average of the signed Alfvenicity (expression (5)) (ACE

This article is protected by copyright. All rights reserved. 
SWEPAM and MAG), the second panel plots the superposed averages of the amplitudes of the outward-sense (red) and inward-sense (blue) Elsasser fluctuations (ACE SWEPAM and MAG), the third panel plots the superposed average of the outward-to-inward Elsasser amplitude, and the bottom panel plots the superposed average of the velocity of the alpha beam relative to the proton velocity (normalized to the proton Alfven speed) (WIND SWE and Mag).

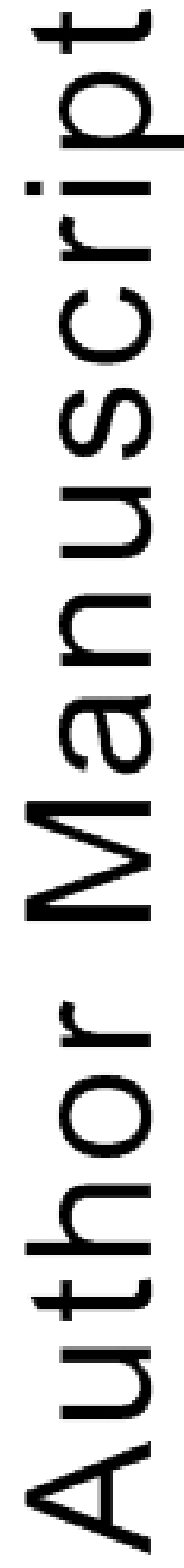



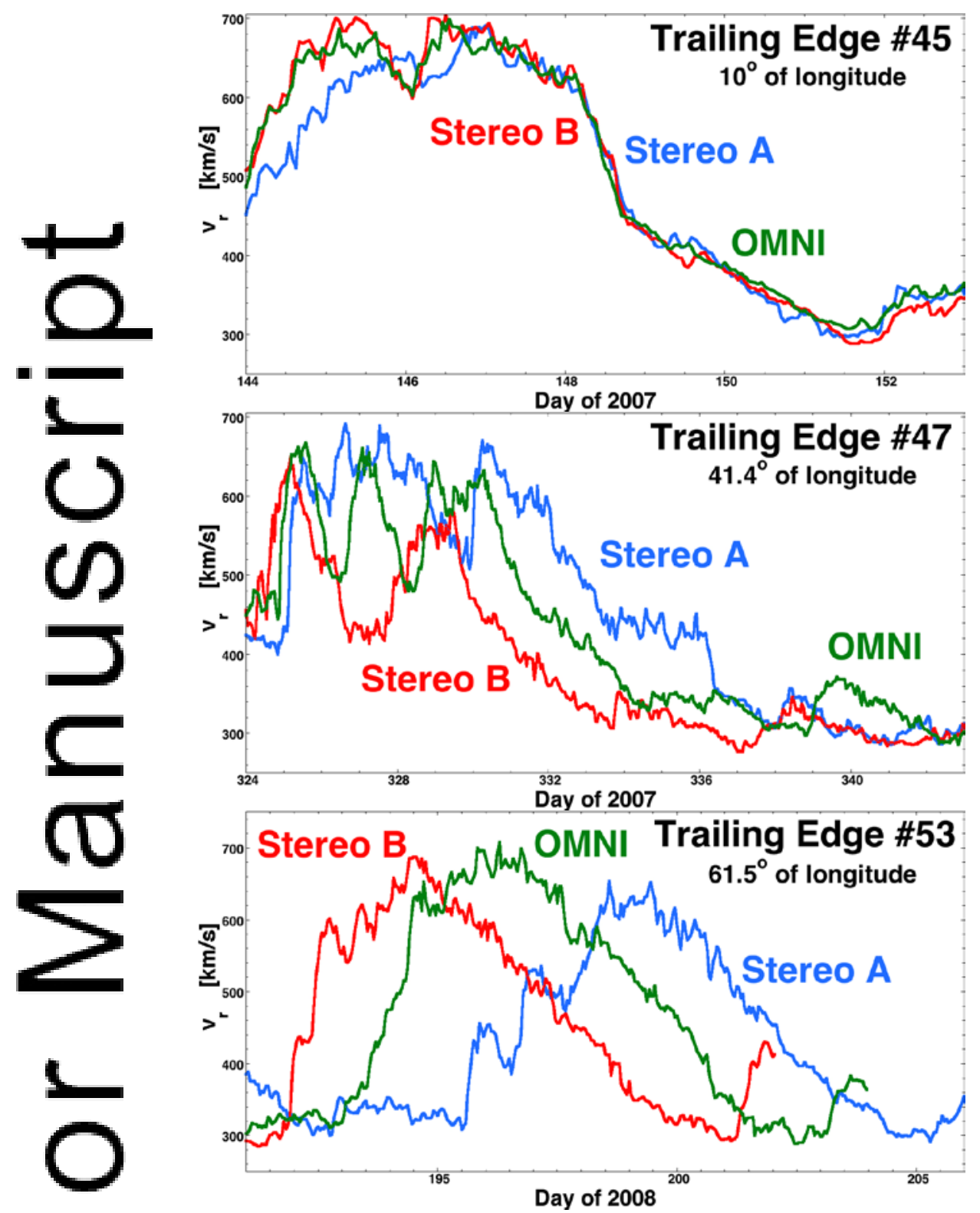

Figure 19. For three trailing edges in the collection of Table 1, the velocity profiles of the trailin rarefactions and the high-speed streams preceding them are examined with the Stereo $A+B$ spacecraft at progressively increasing distances ahead and behind the Earth.

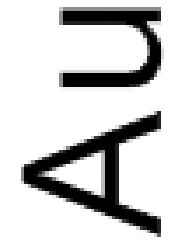

This article is protected by copyright. All rights reserved. 

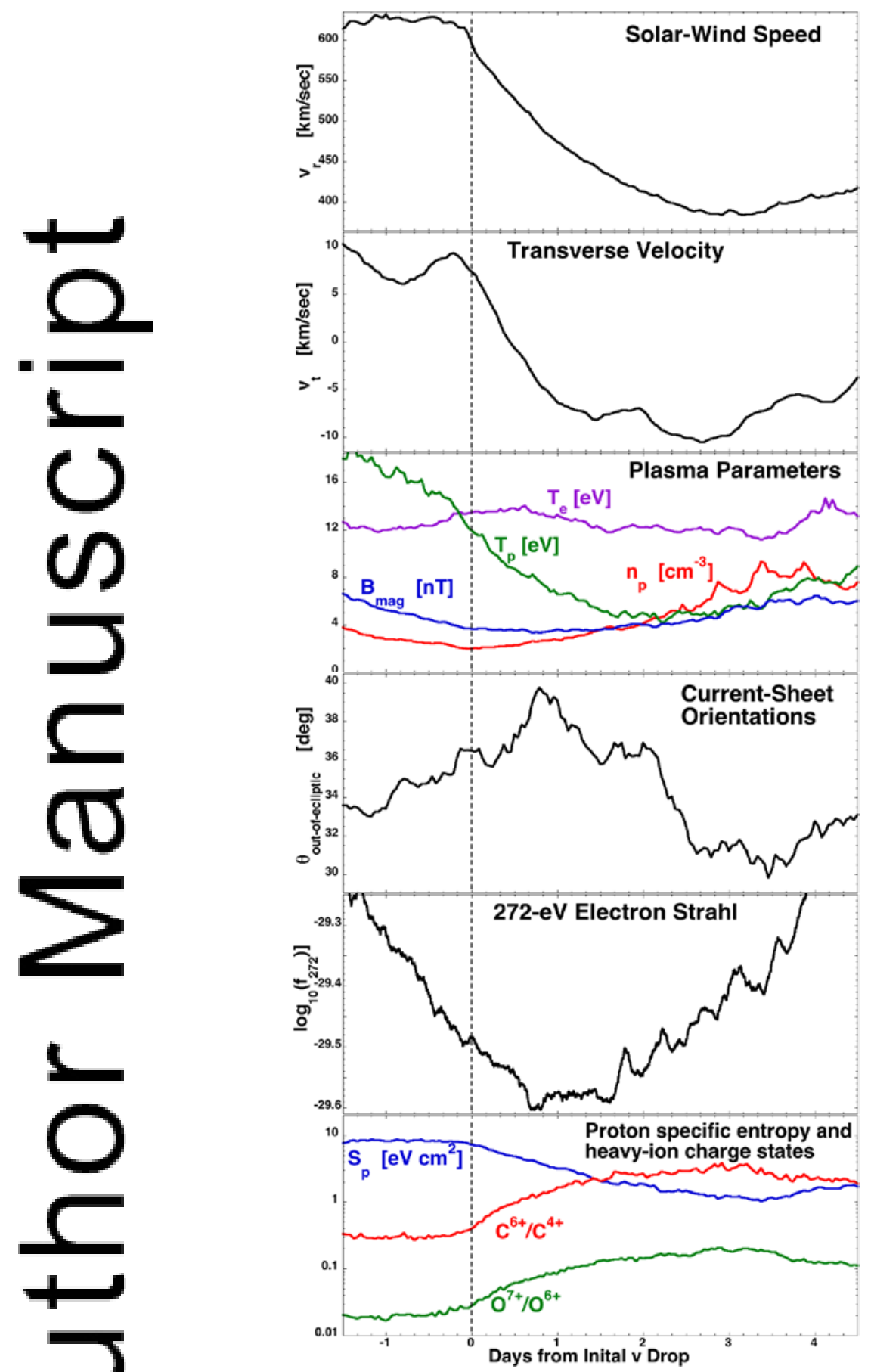

Figure Superposed averages of measurements for the 54 trailing edges of Table 1 are plotted. The zero epoch rtical dashed line) is the passage of the initial velocity drop in each trailing edge. Plotted in the topa hel is the solar-wind speed (OMNI), in the second panel the transverse velocity of the solar 
wind (OMNI), in the third panel the plasma number density (OMNI), proton temperature (OMNI), magnetic-field strength (OMNI), and electron temperature (Wind 3DP), in the fourth panel the out-ofecliptic angles of the current-sheet-normal vectors (ACE MAG), in the fifth panel intensity of the 272-eV electron strahl (ACE SWEPAM), and in the bottom panel the proton specific entropy (OMNI), the carbon charge-state-density ratio (ACE SWICS), and the oxygen charge-state ratio (ACE SWICS).

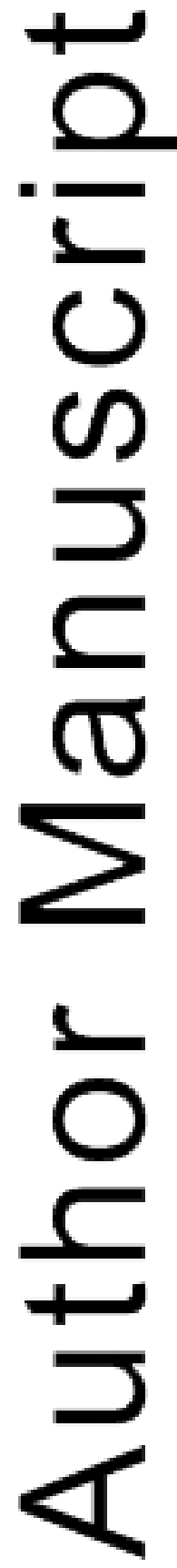




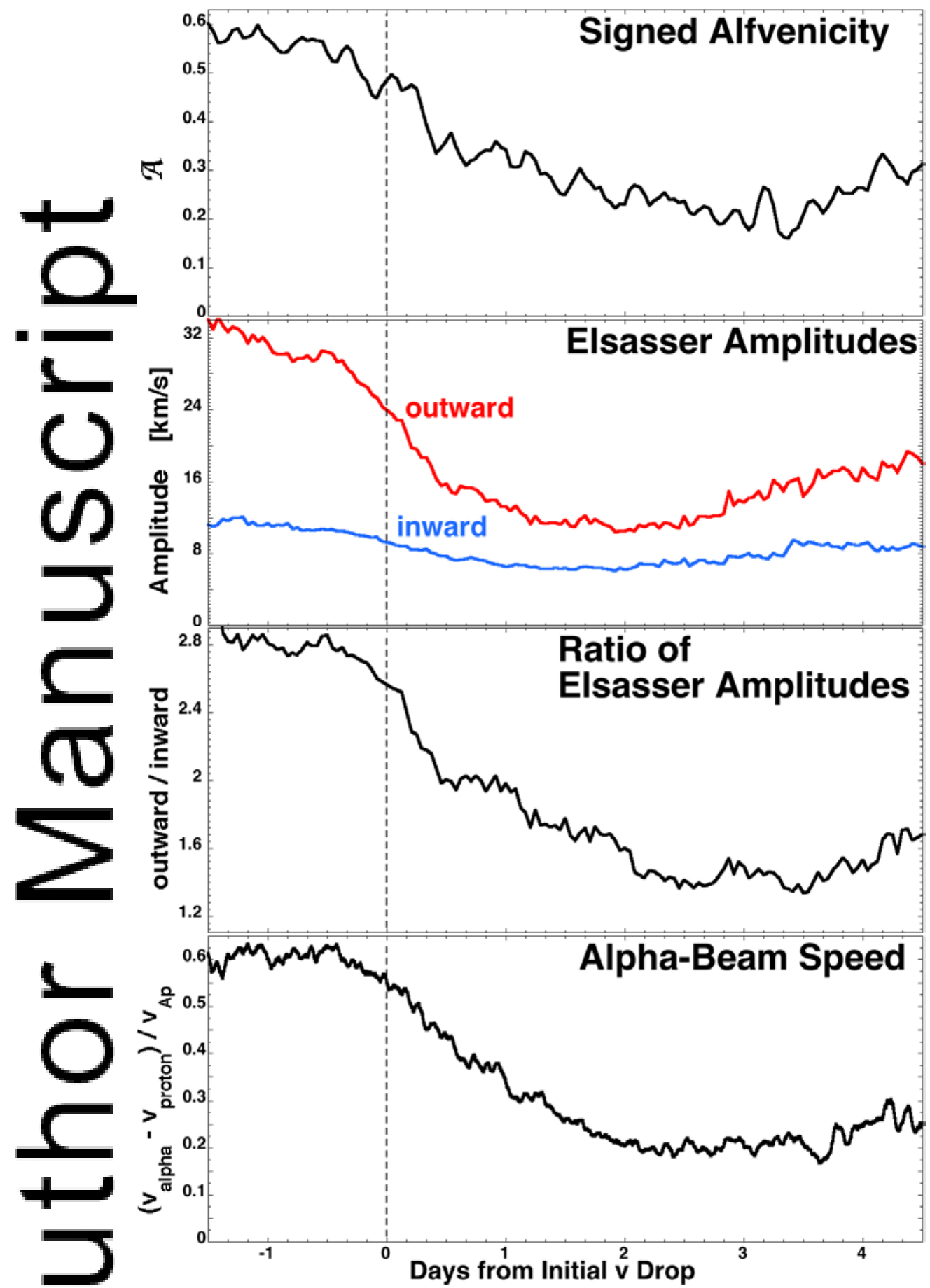

Figure 21. Triggered on the velocity-bend of the 54 trailing edges in Table 1, the top panel plots the suer osed average of the signed Alfvenicity (expression (5)) (ACE SWEPAM and MAG),

This article is protected by copyright. All rights reserved. 
the second panel plots the superposed averages of the amplitudes of the outward-sense (red) and inward-sense (blue) Elsasser fluctuations (ACE SWEPAM and MAG), the third panel plots the superposed average of the outward-to-inward Elsasser amplitude, and the bottom panel plots the superposed average of the velocity of the alpha beam relative to the proton velocity (normalized to the proton Alfven speed) (WIND SWE and Mag).

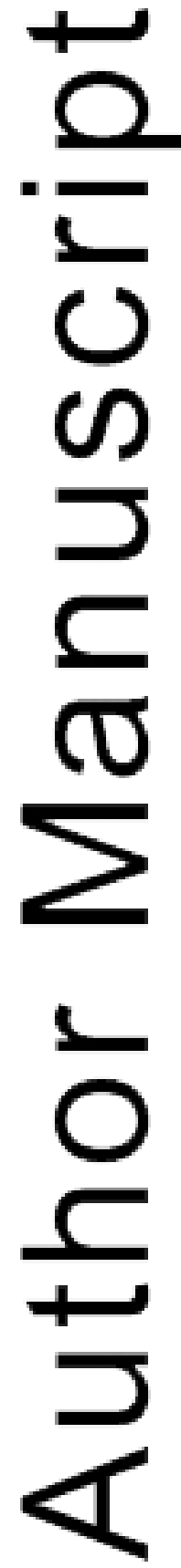




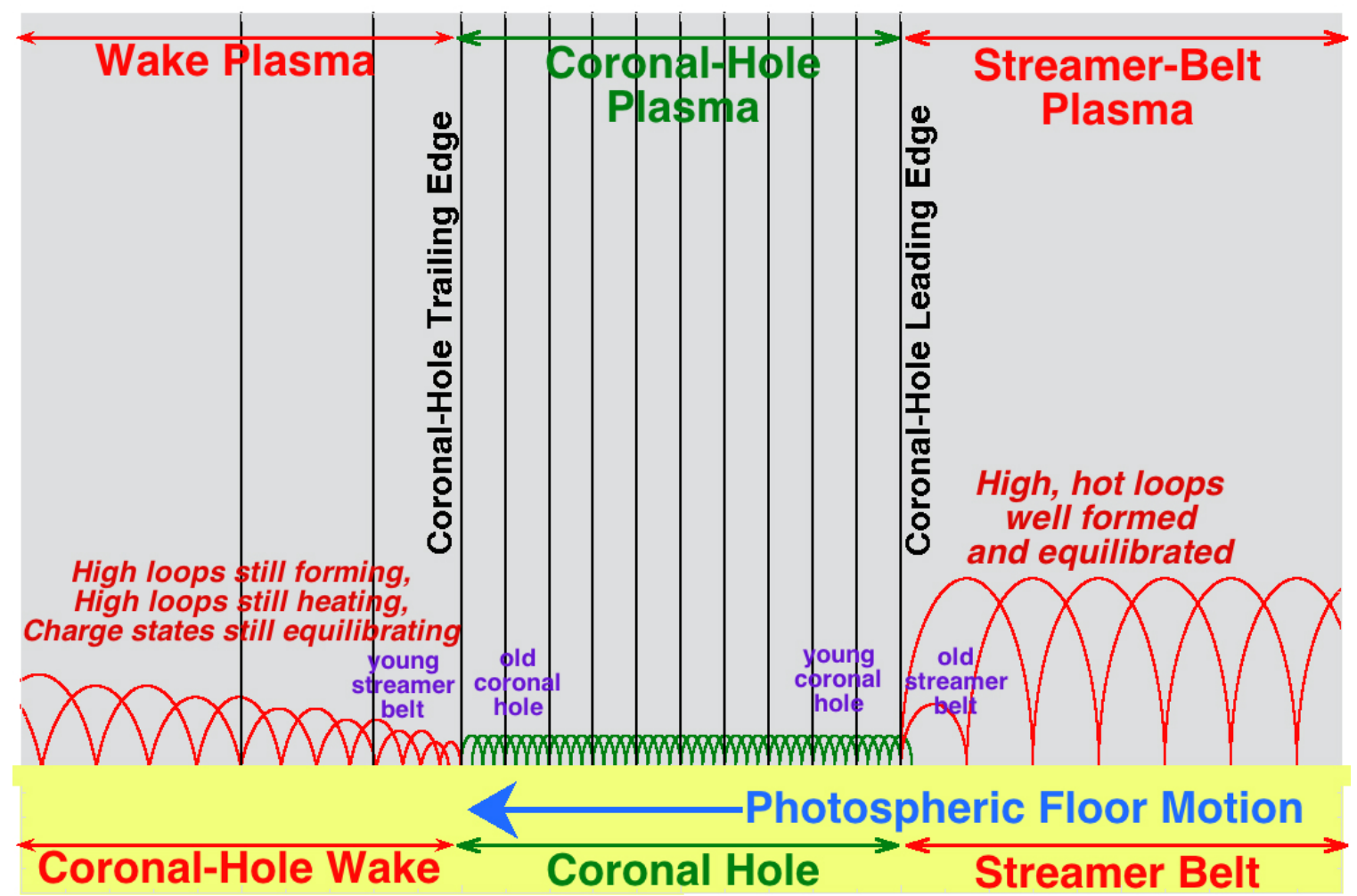

Figure 20 sketch of the open flux of a coronal hole (with low-lying closed loops (green) on the coln 1 hole floor) sandwiched between two regions of streamer belt (with higher closed loops (red)). In the reference frame of the coronal hole and its open flux, the photosphere (yellow moves from right to left so that photosphere enters the coronal hole on the leading edge and exits coronal hole on the trailing edge. It is hypothesized that the transition from open flux cured flux at the trailing-edge boundary might have a temporal wake wherein it takes 10 's of hours to form high closed loops, to heat the high loops, and to reach charge-state equilibrium in the high loops.

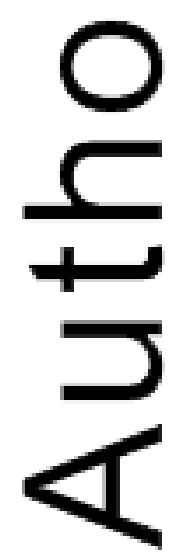




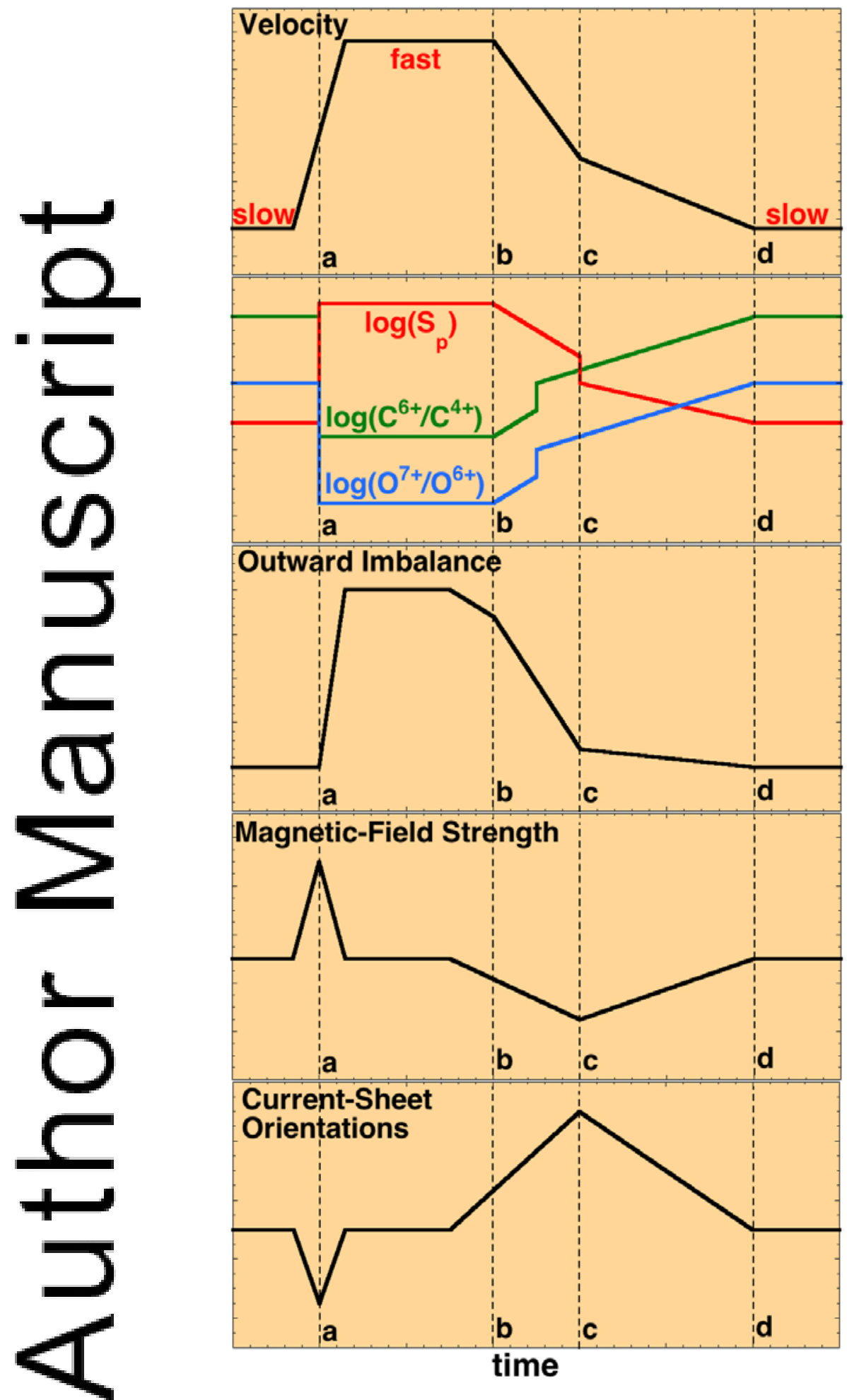

This article is protected by copyright. All rights reserved. 
Figure 23. A working sketch to organize the timing of the signatures in a high-speed stream, its leading-edge CIR compression, and its trailing-edge rarefaction. The four vertical dashed lines are $\mathrm{a}=$ leading-edge $(\mathrm{CIR})$ stream interface, $\mathrm{b}=$ initial velocity drop, $\mathrm{c}=$ velocity-bend (trailingedge stream interface),$d=$ velocity termination of trailing edge.

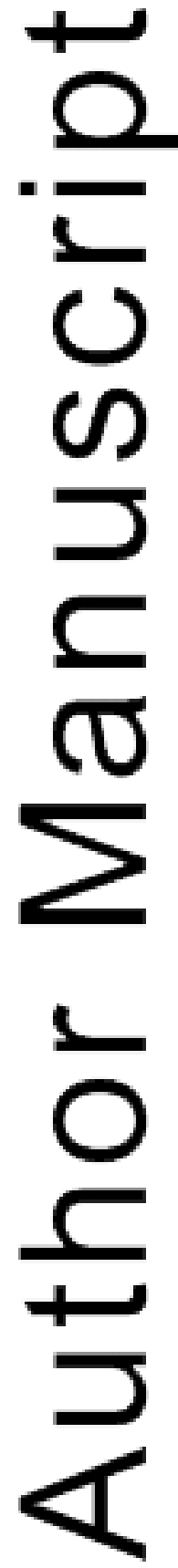




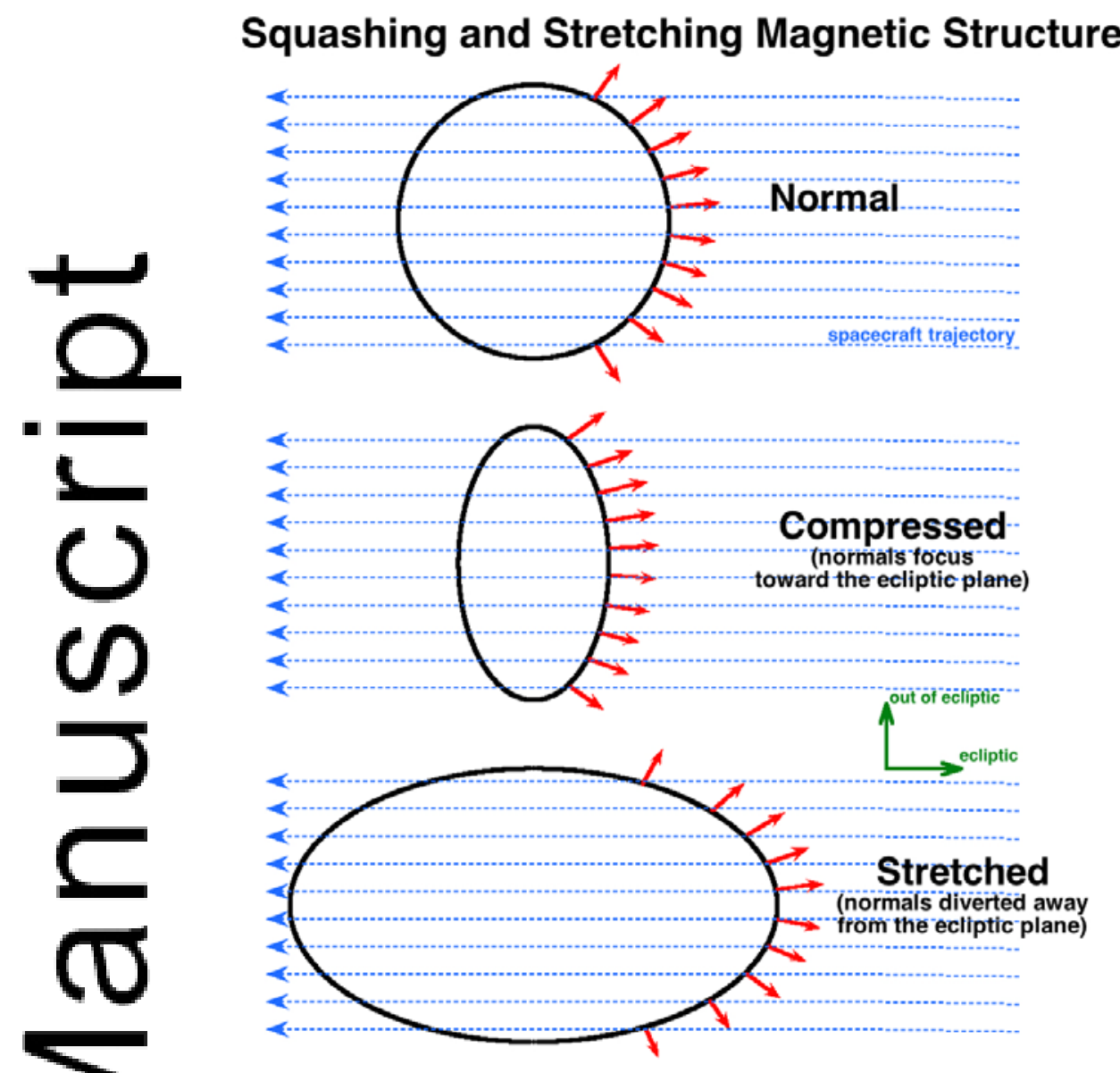

Figure 24 or a round flux tube (top), a compressed flux tube (middle), and a rarefacted flux tube mans , red arrows mark the orientations of the normal vectors to the current sheet that bounds the flux tube. The coordinate system is indicated in green.

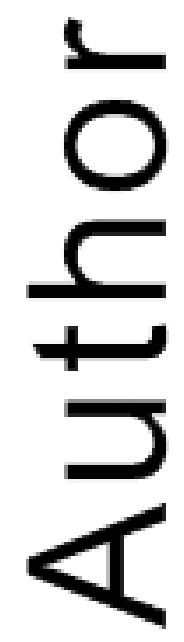

This article is protected by copyright. All rights reserved. 


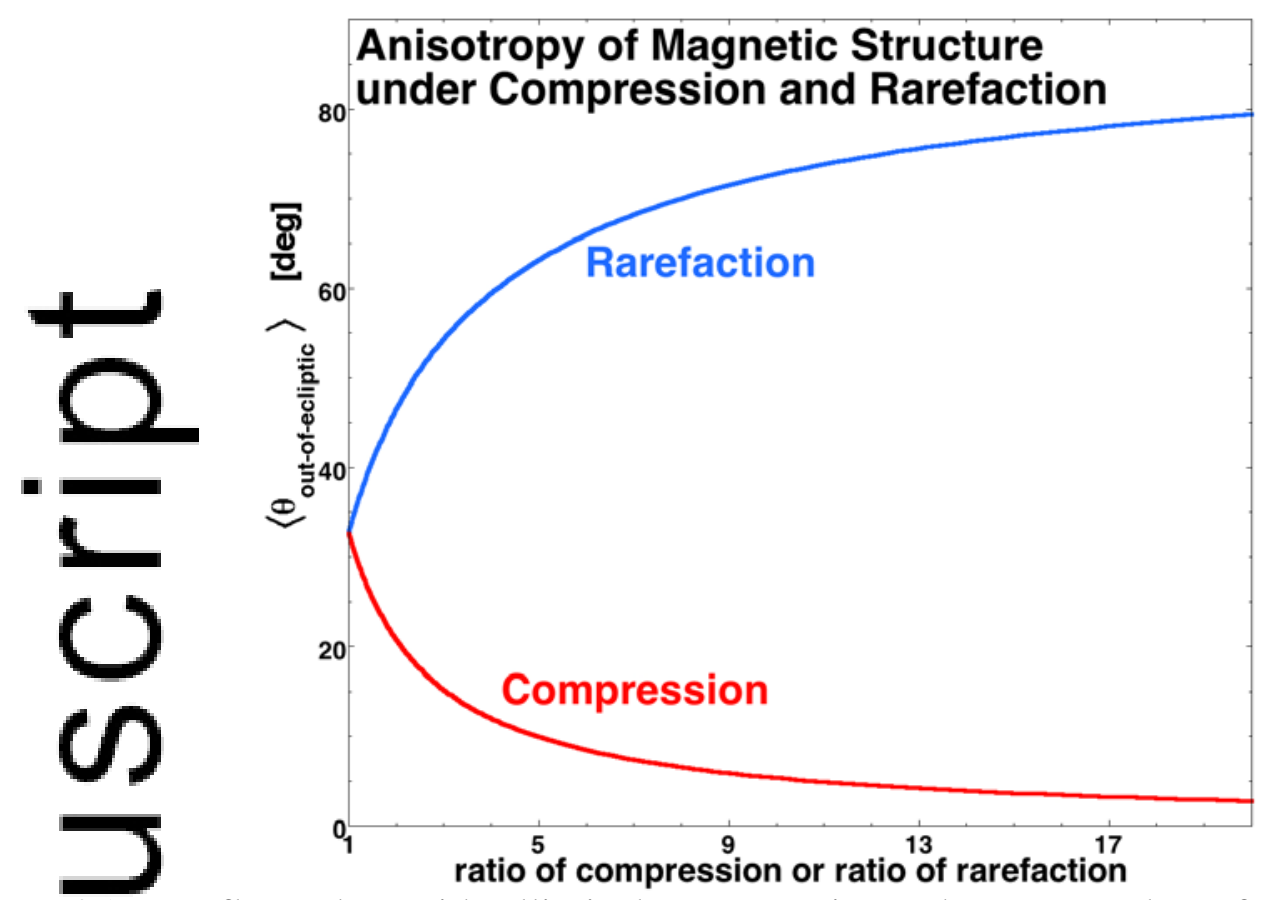

Figure 25. For flux tubes with elliptical cross sections, the mean value of the out-of-ecliptic angle fo current-sheet normal vectors of the flux-tube walls are numerically calculated and plotted as a function of the amount of compression (red) of the flux tubes or rarefaction (blue) of the fluy (10).
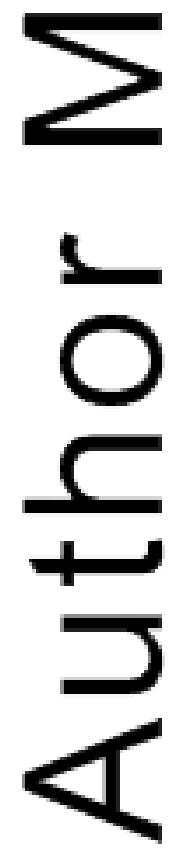

This article is protected by copyright. All rights reserved. 


\section{Leading-Edge Compression}

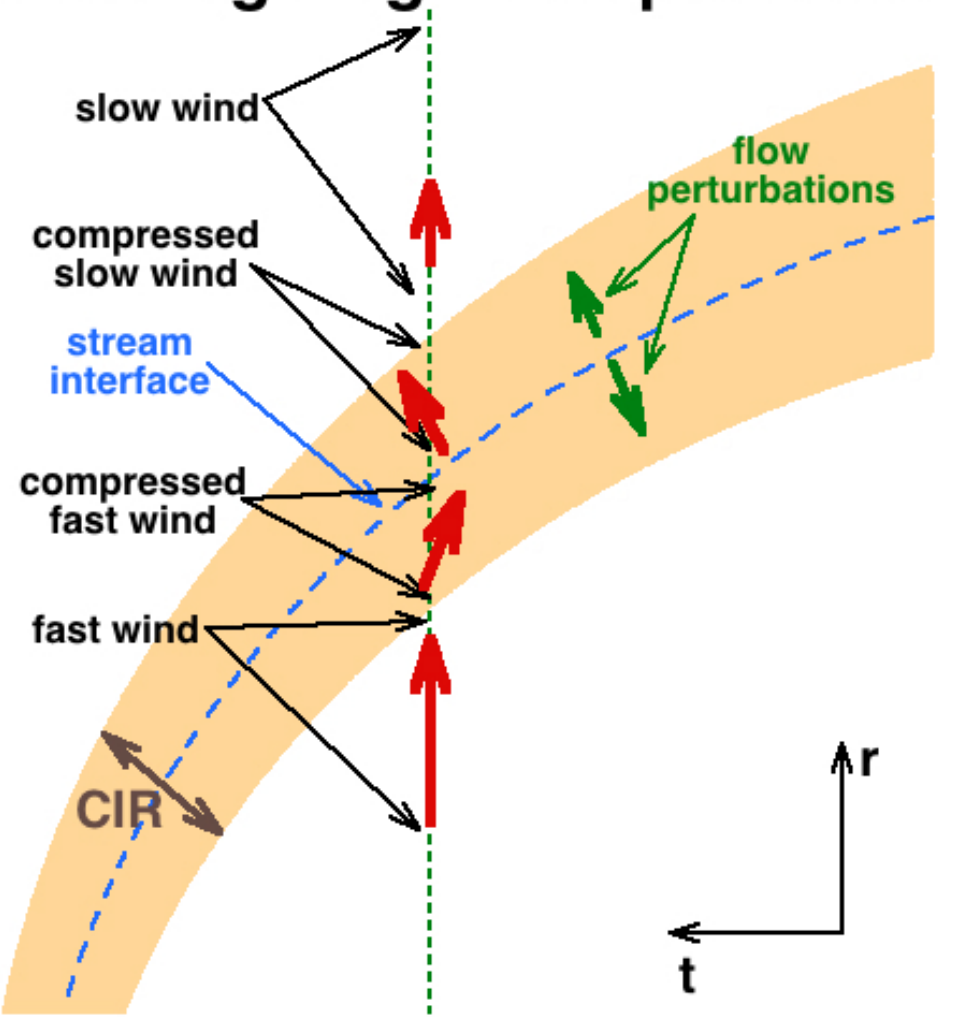

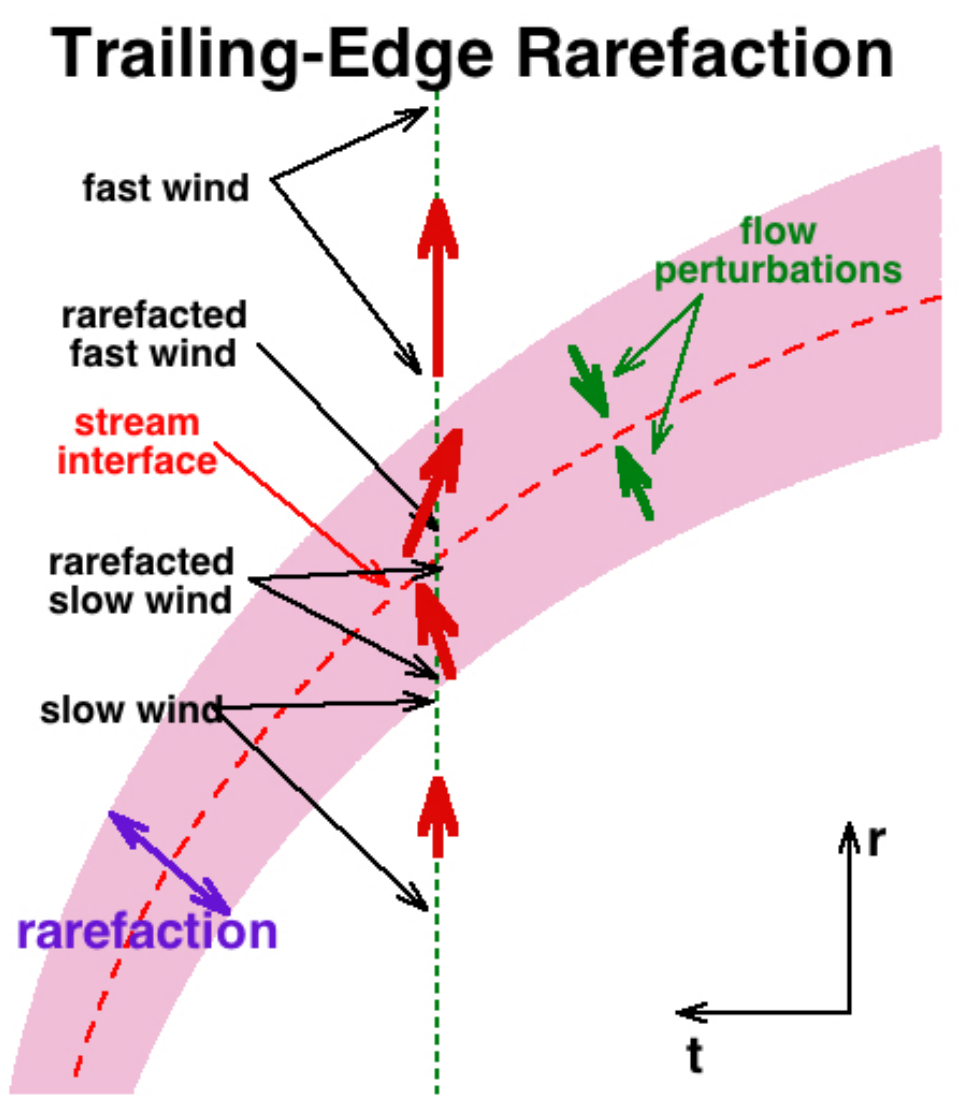




\section{Leading-Edge Stream Interface}

Positive Vorticity $\omega$

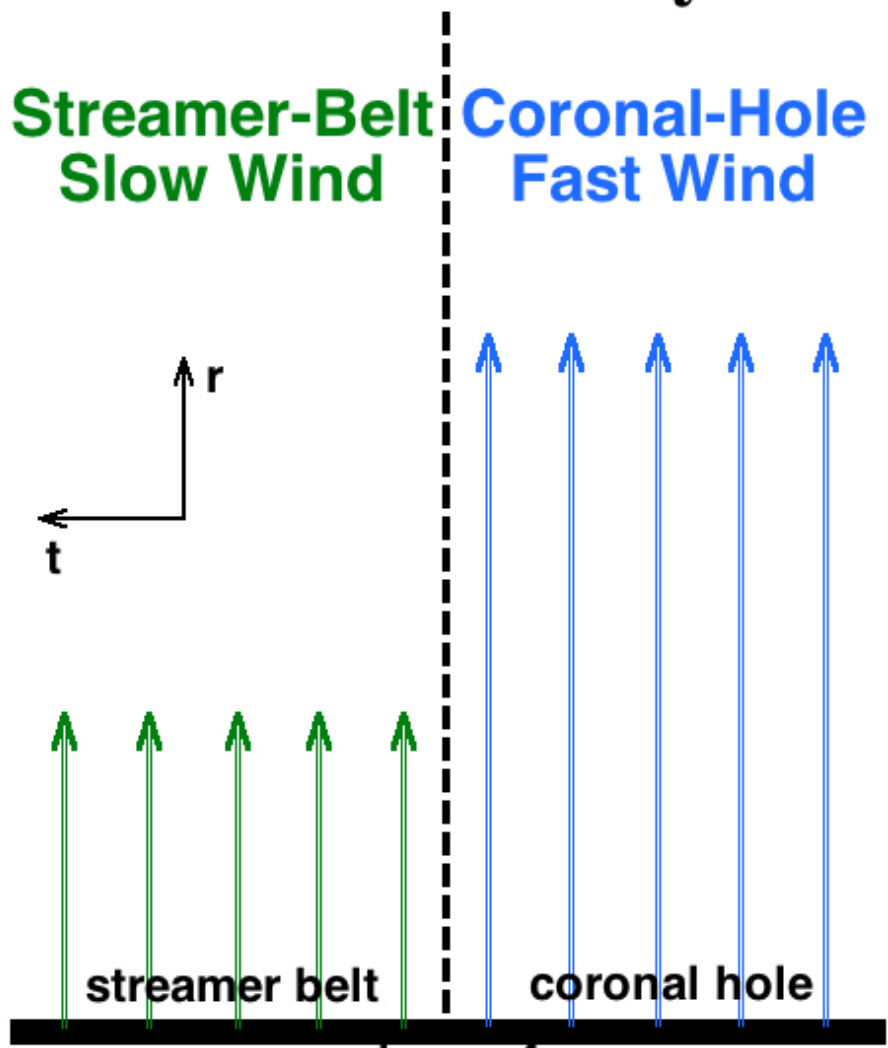

solar surface

direction of solar rotation

\section{Trailing-Edge Stream Interface}

Negative Vorticity $\omega$

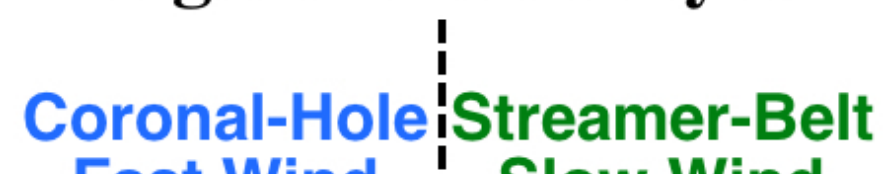
Fast Wind Slow Wind

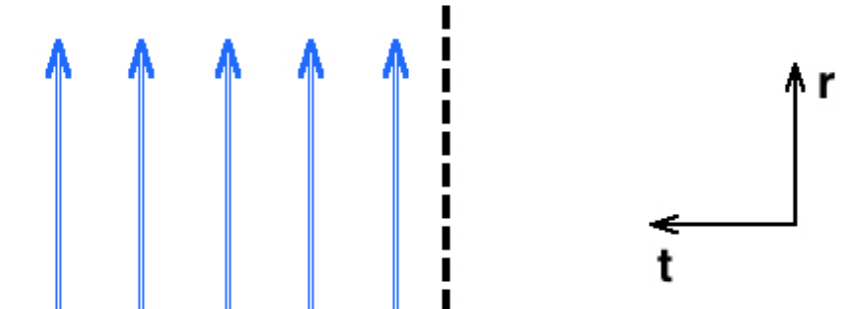

coronal hole streamer belt solar surface

direction of solar rotation 


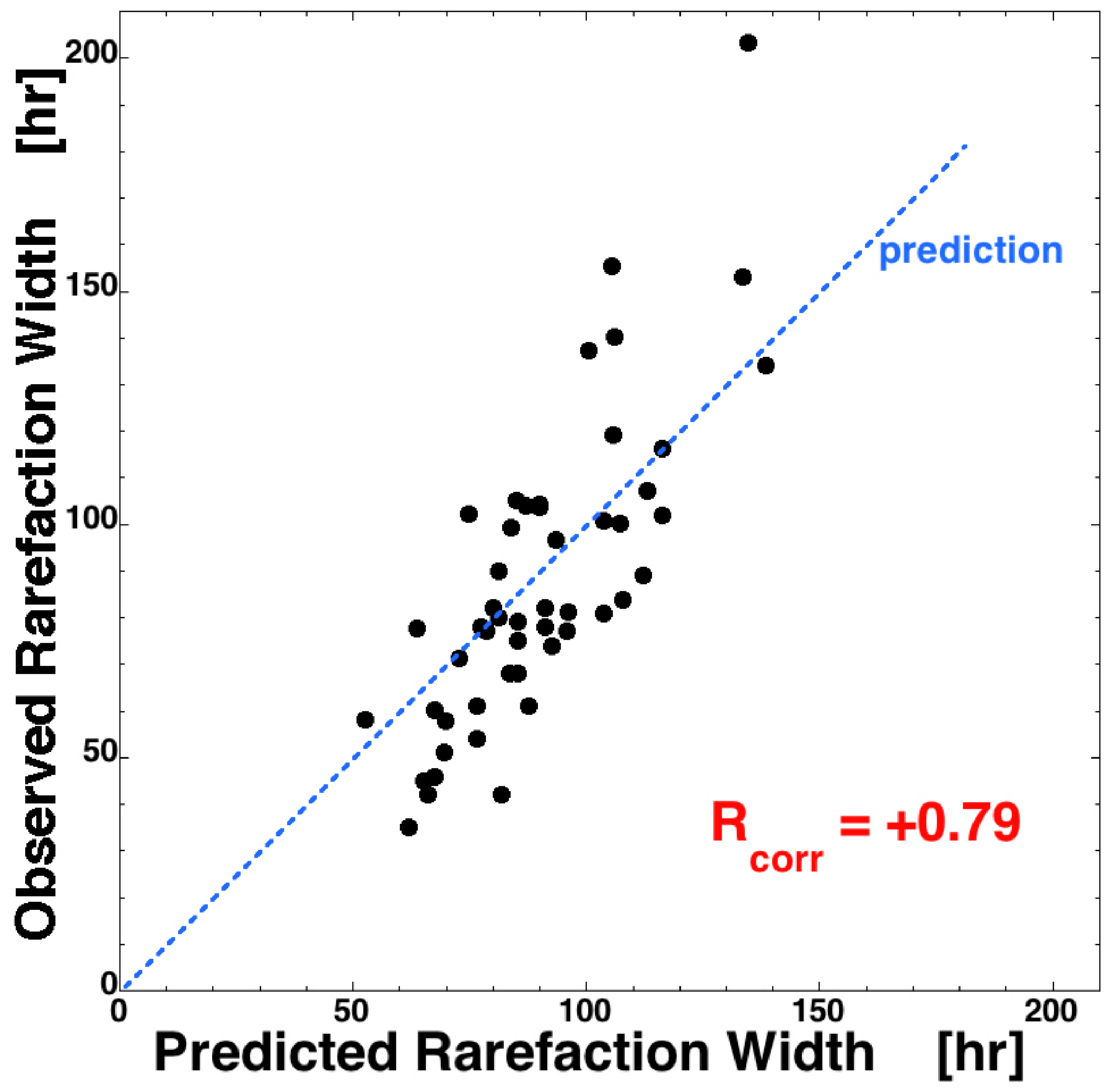

2016JA022863-f06-z-.jpg 

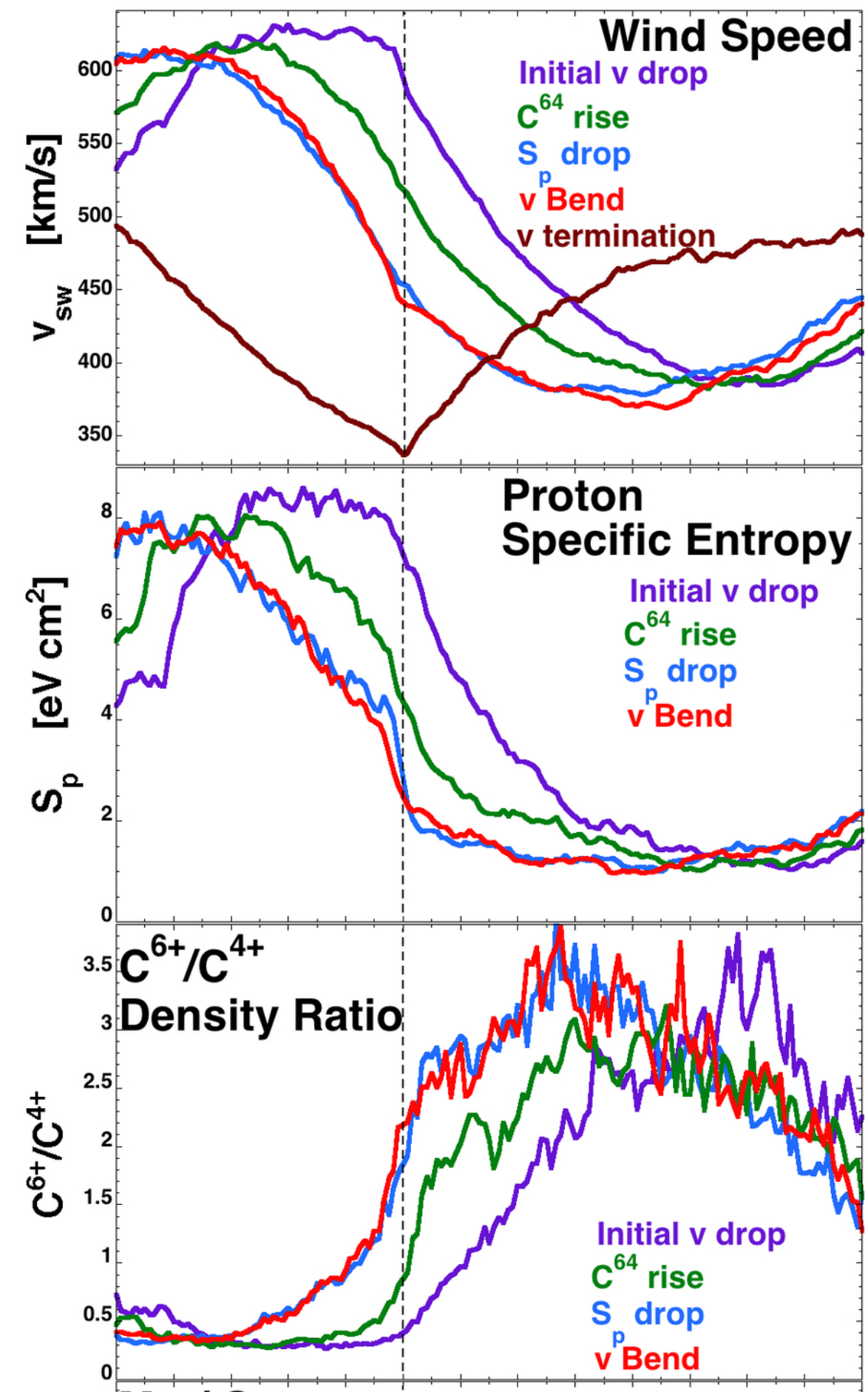

${ }^{0.15} \mathrm{Mg} / \mathrm{O}$
Density Ratio

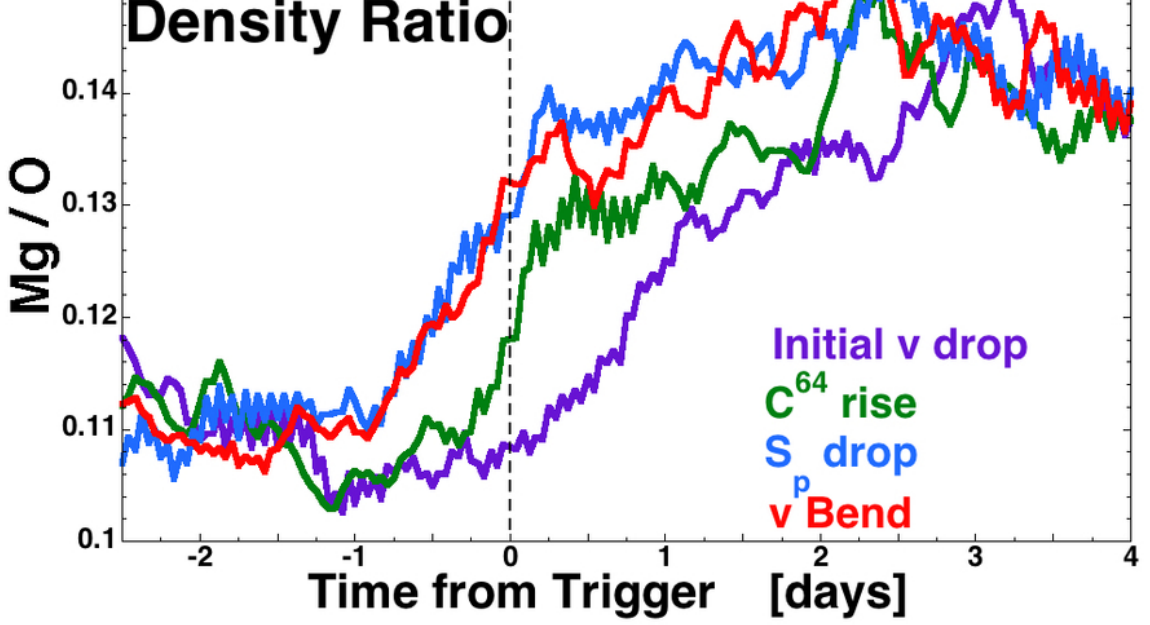




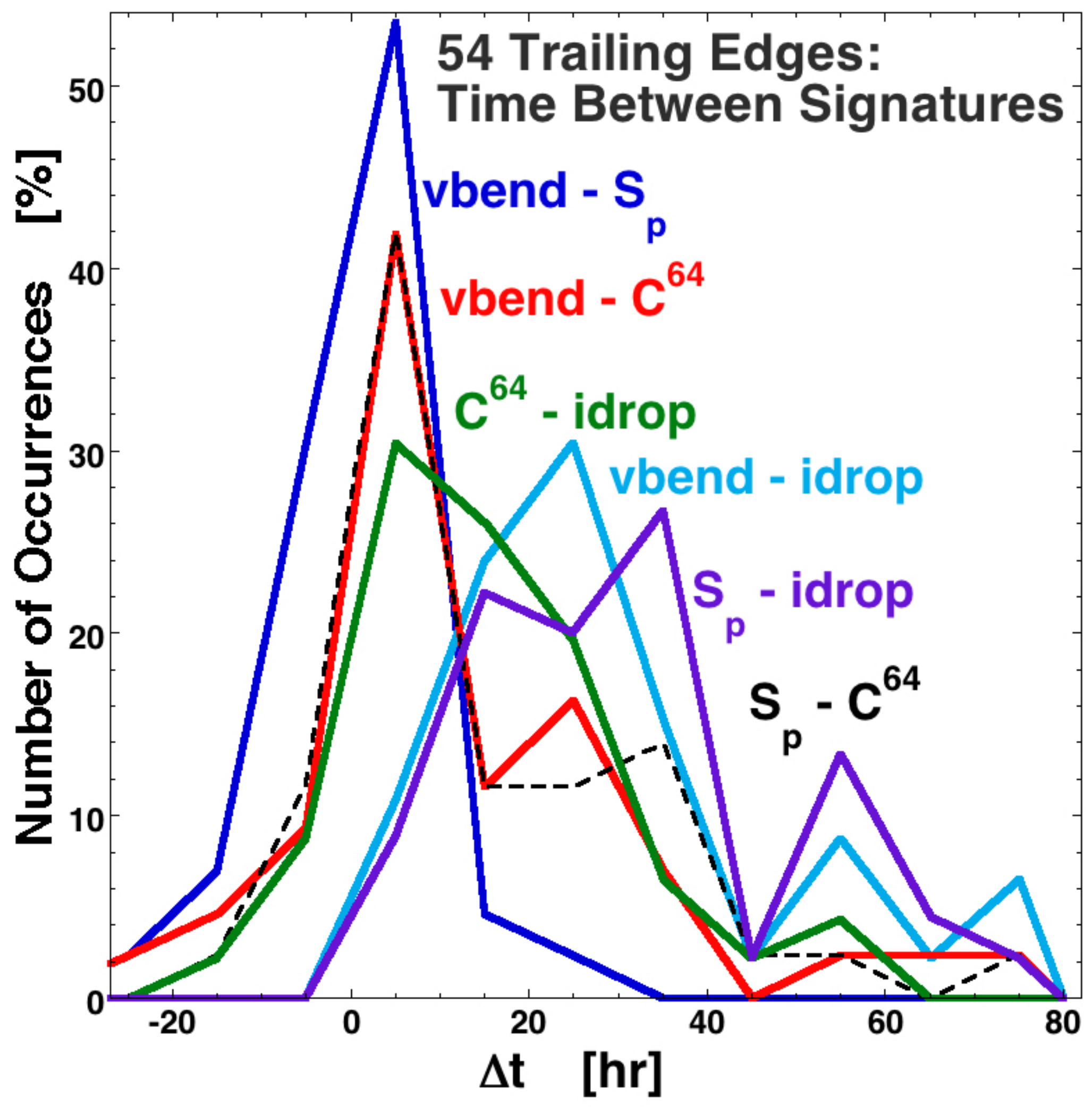

2016JA022863-f08-z-.jpg 


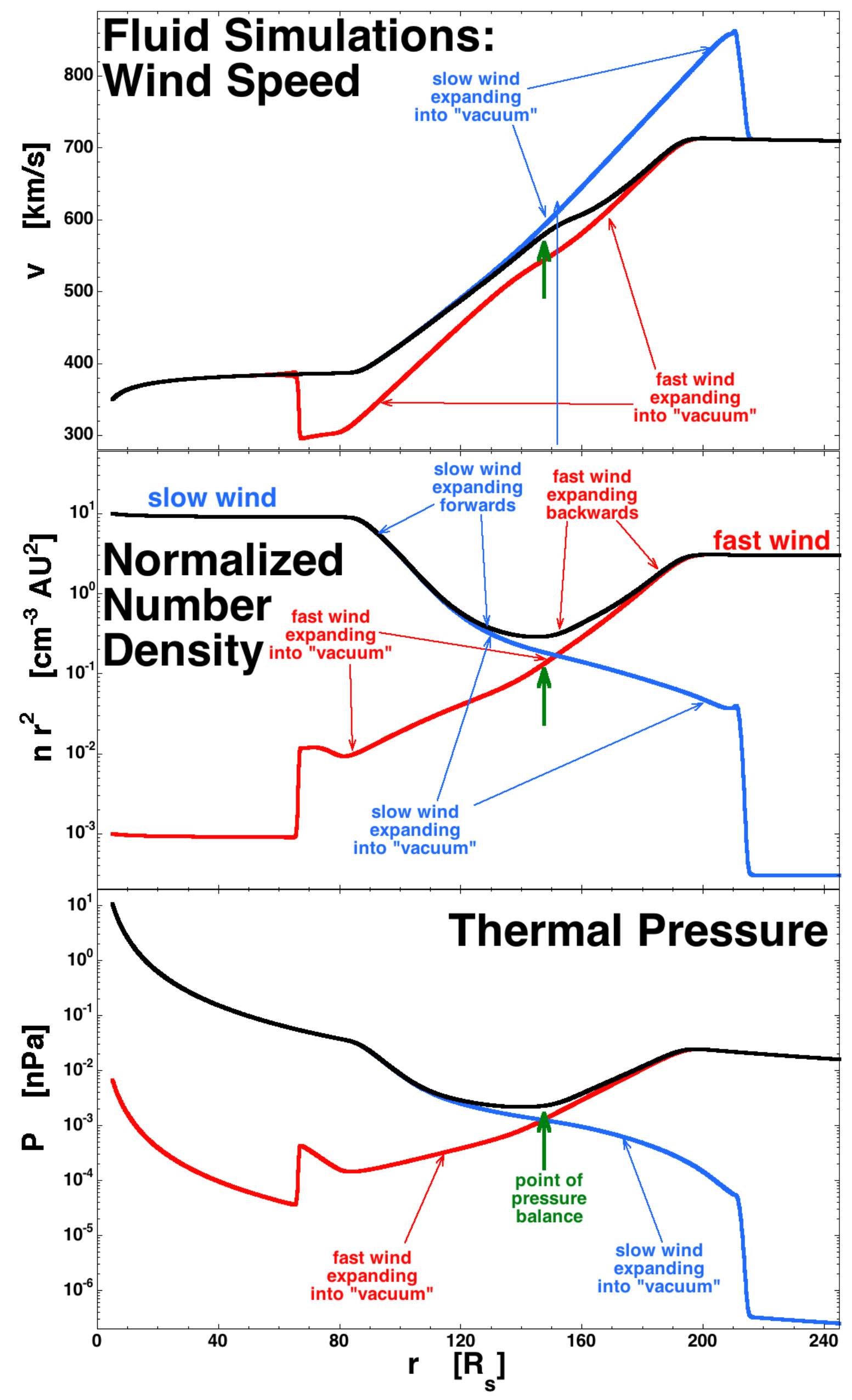




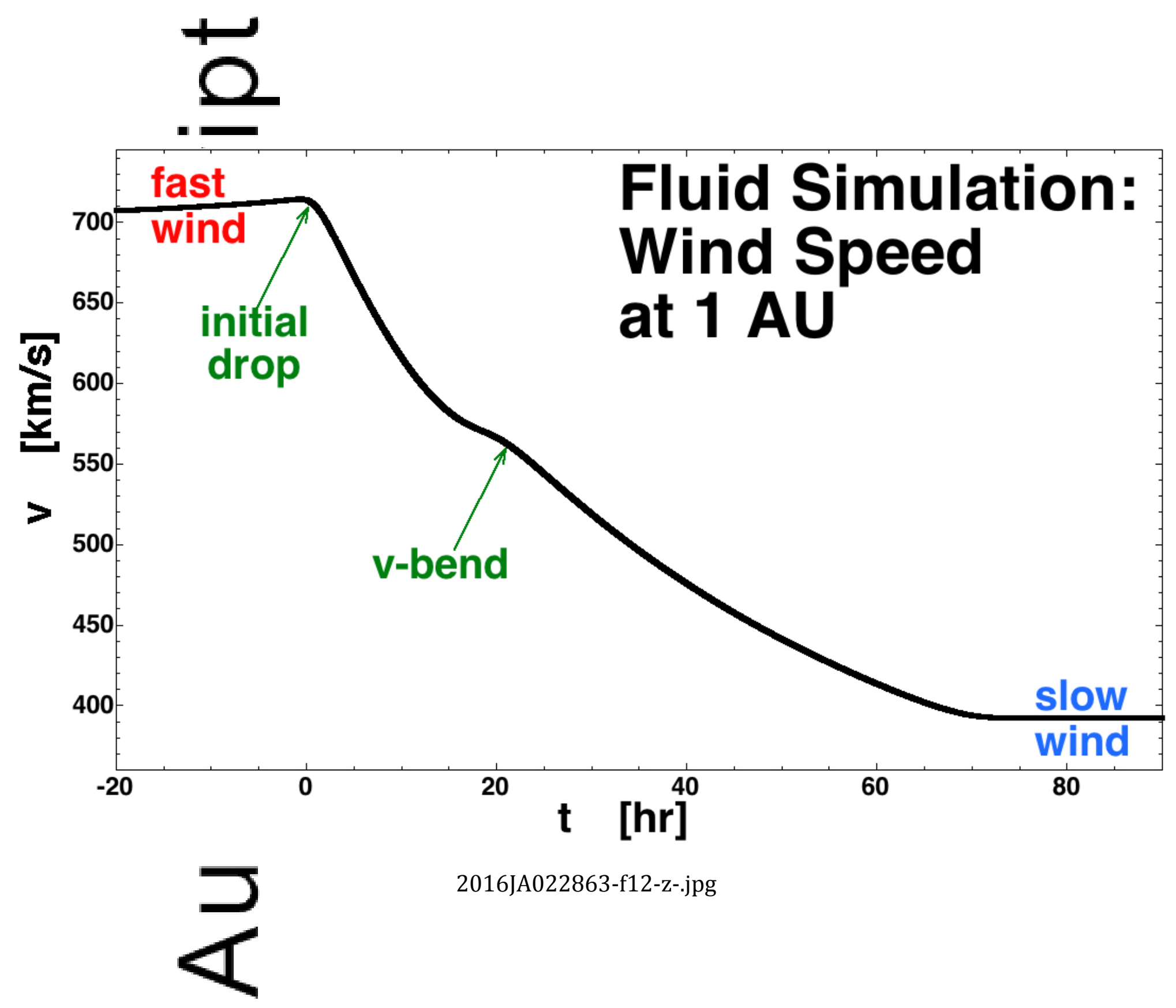



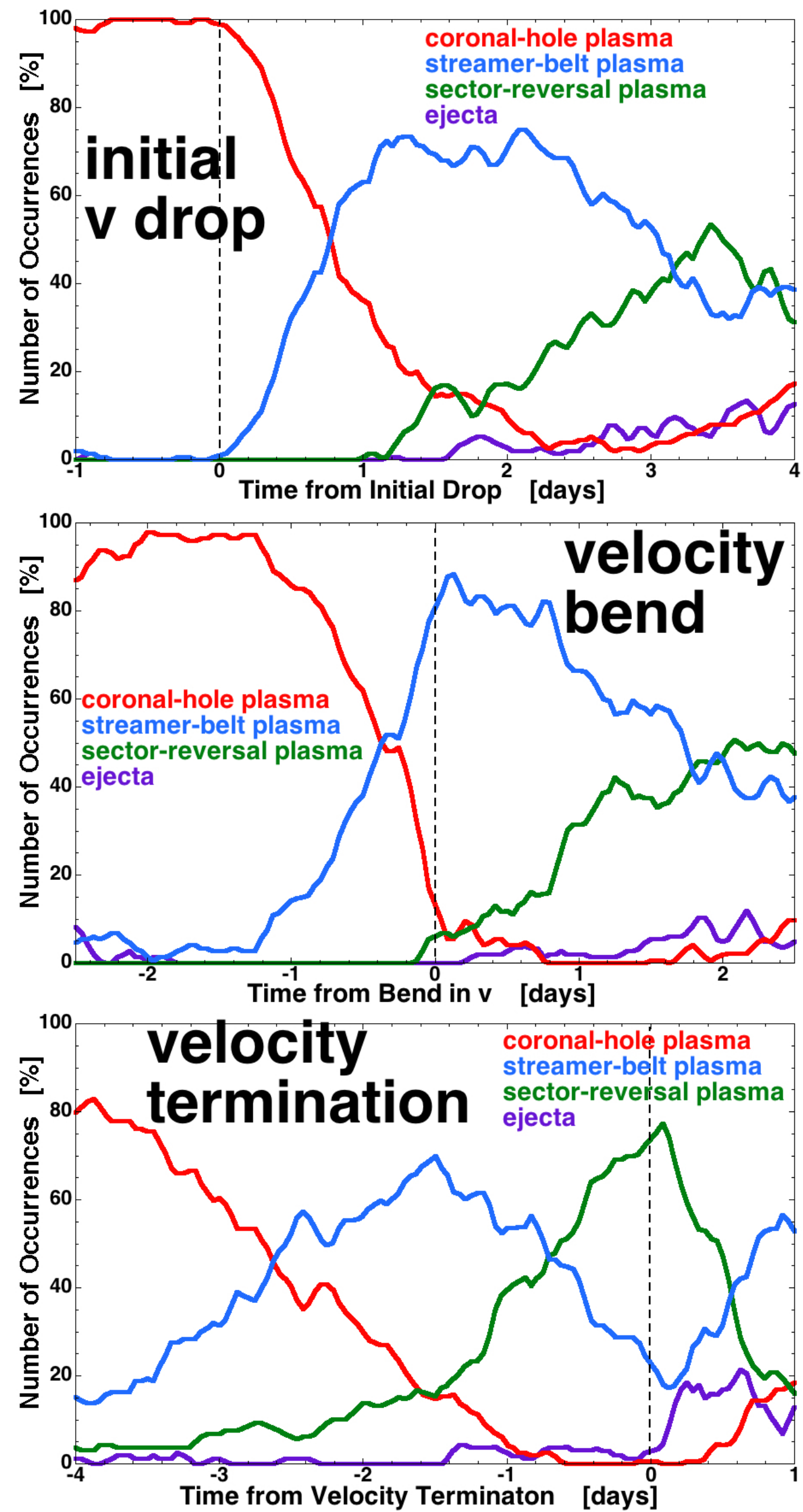


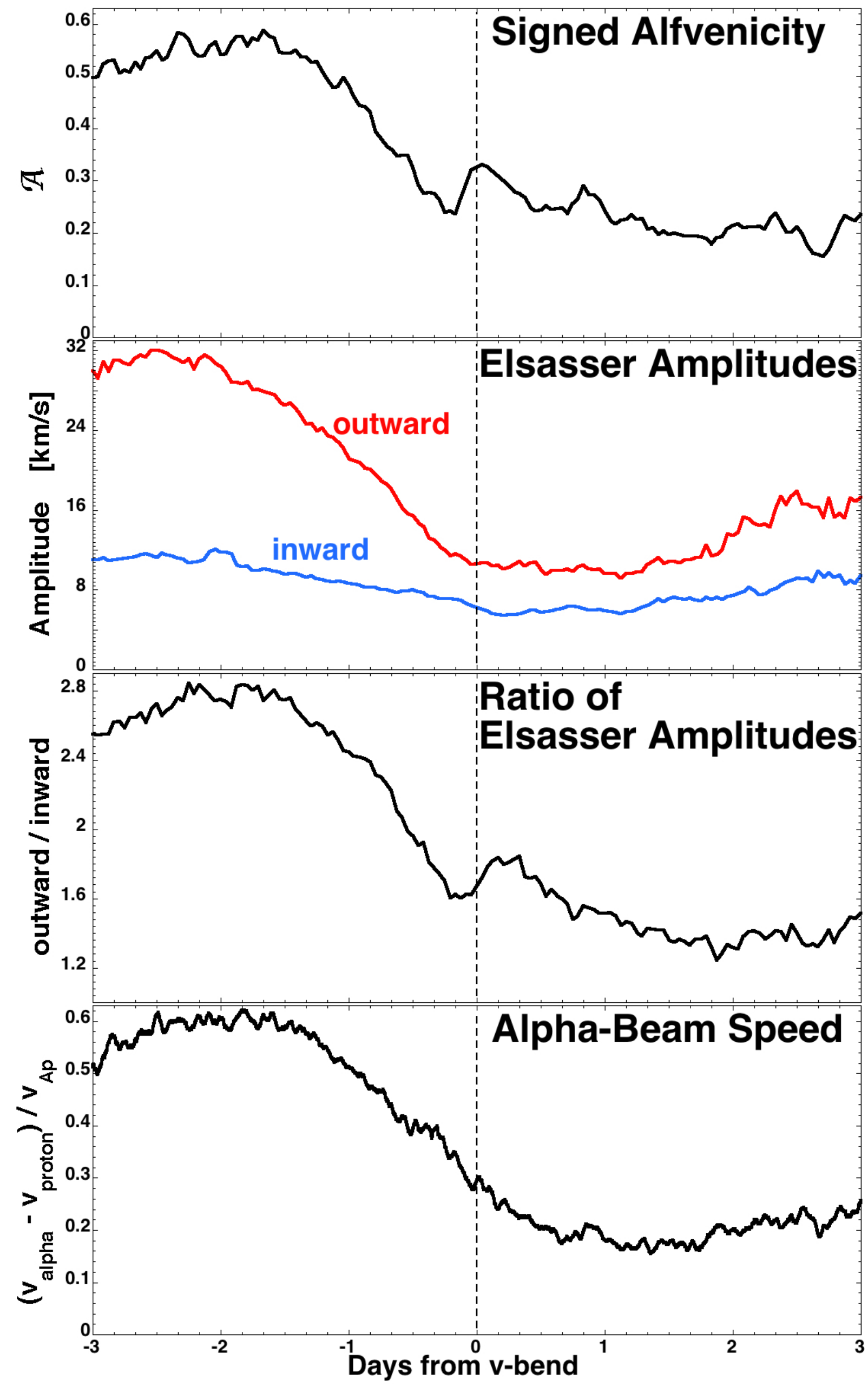




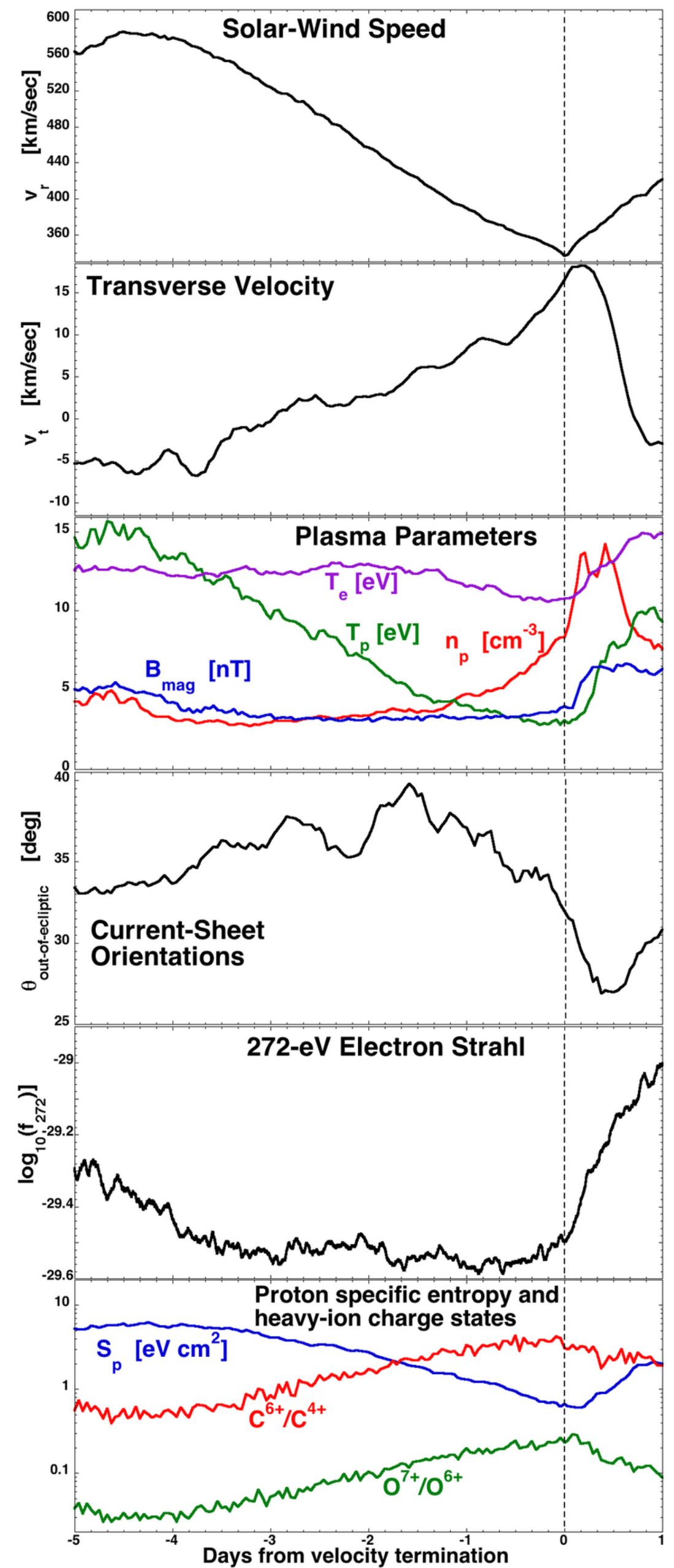




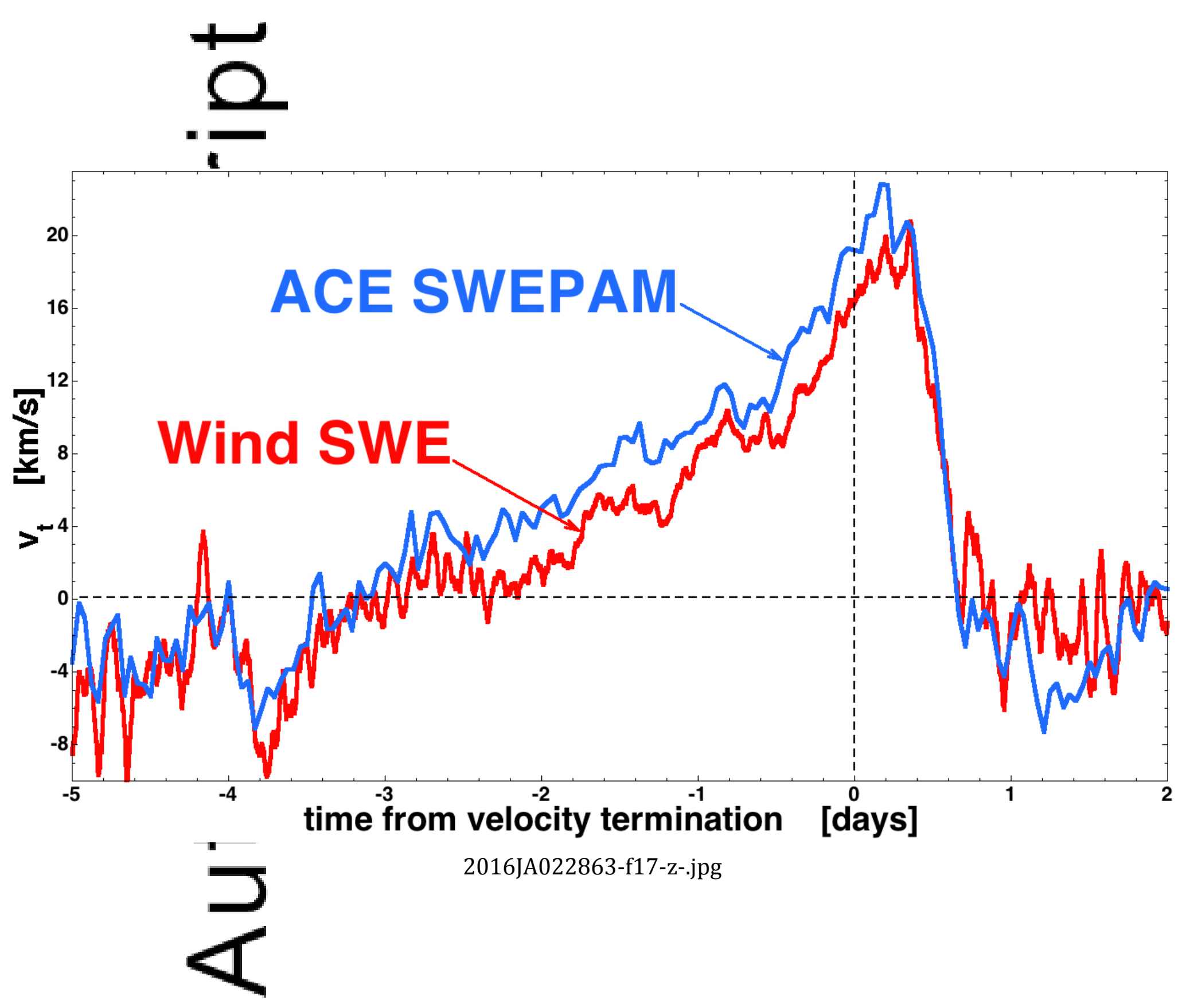

This article is protected by copyright. All rights reserved. 

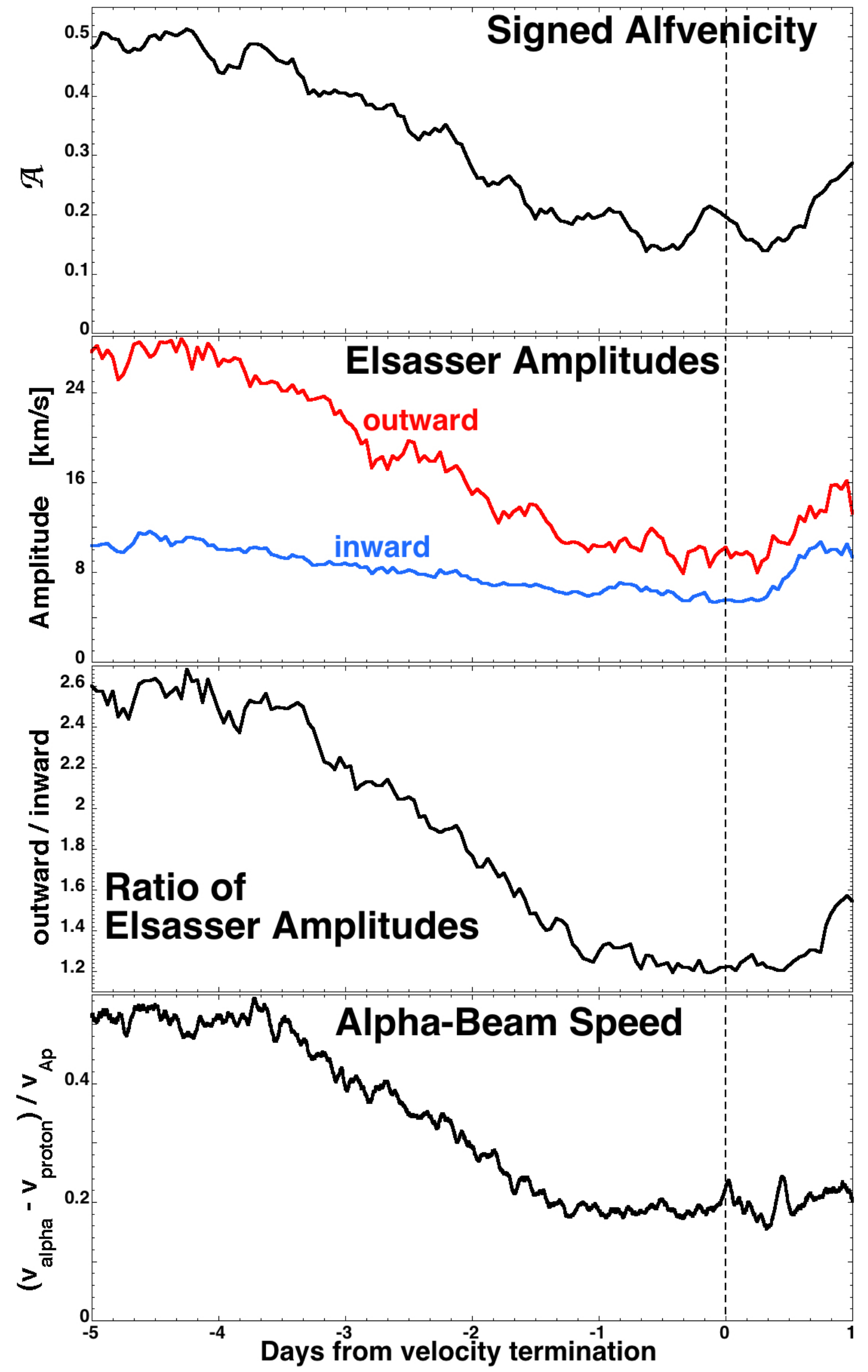


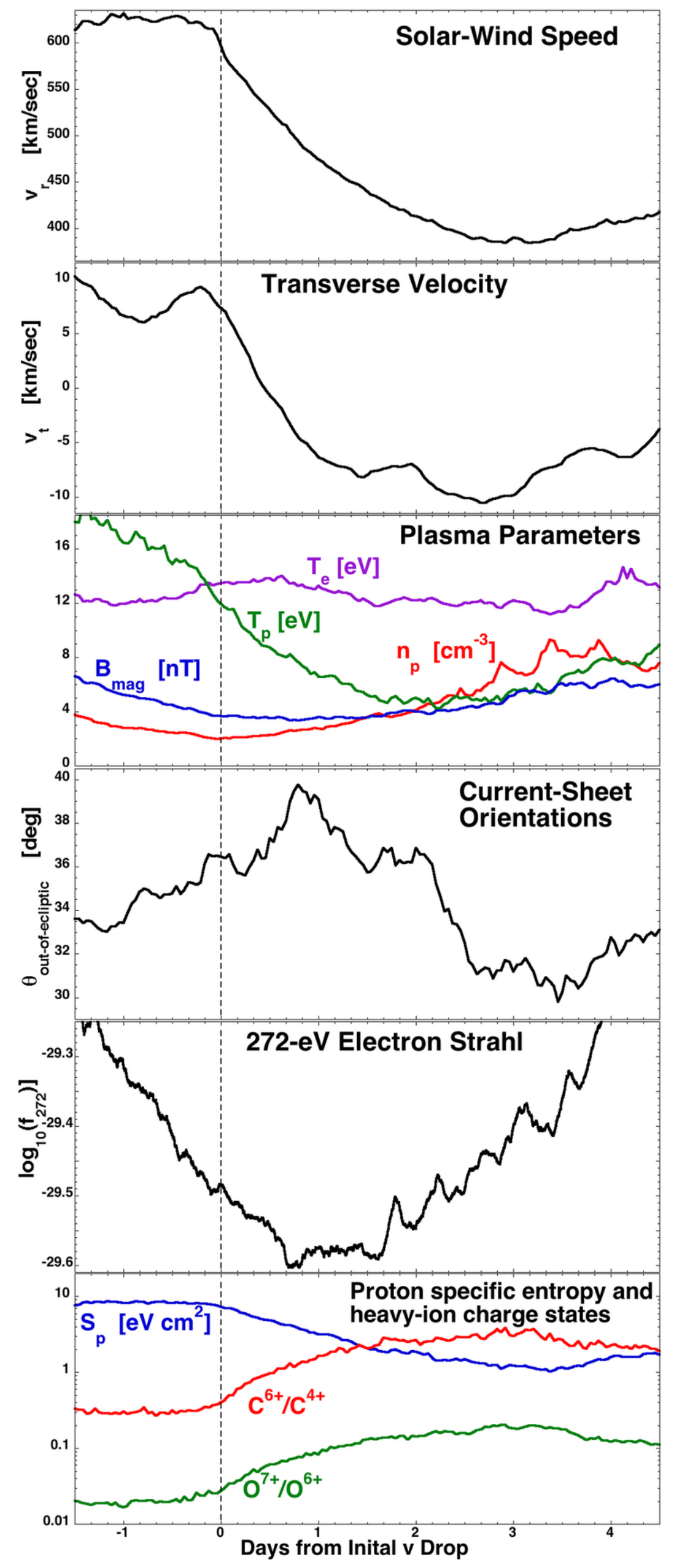




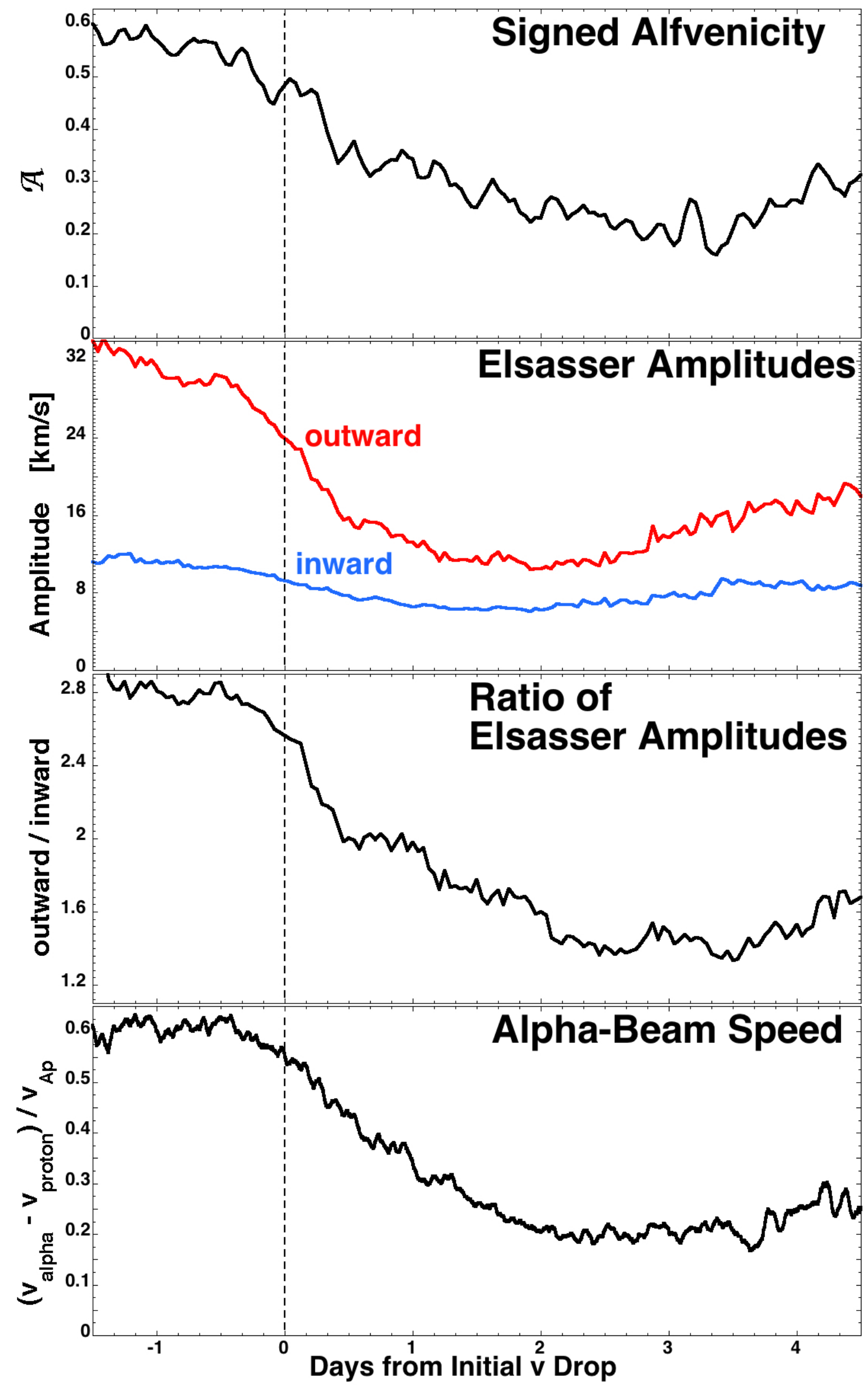




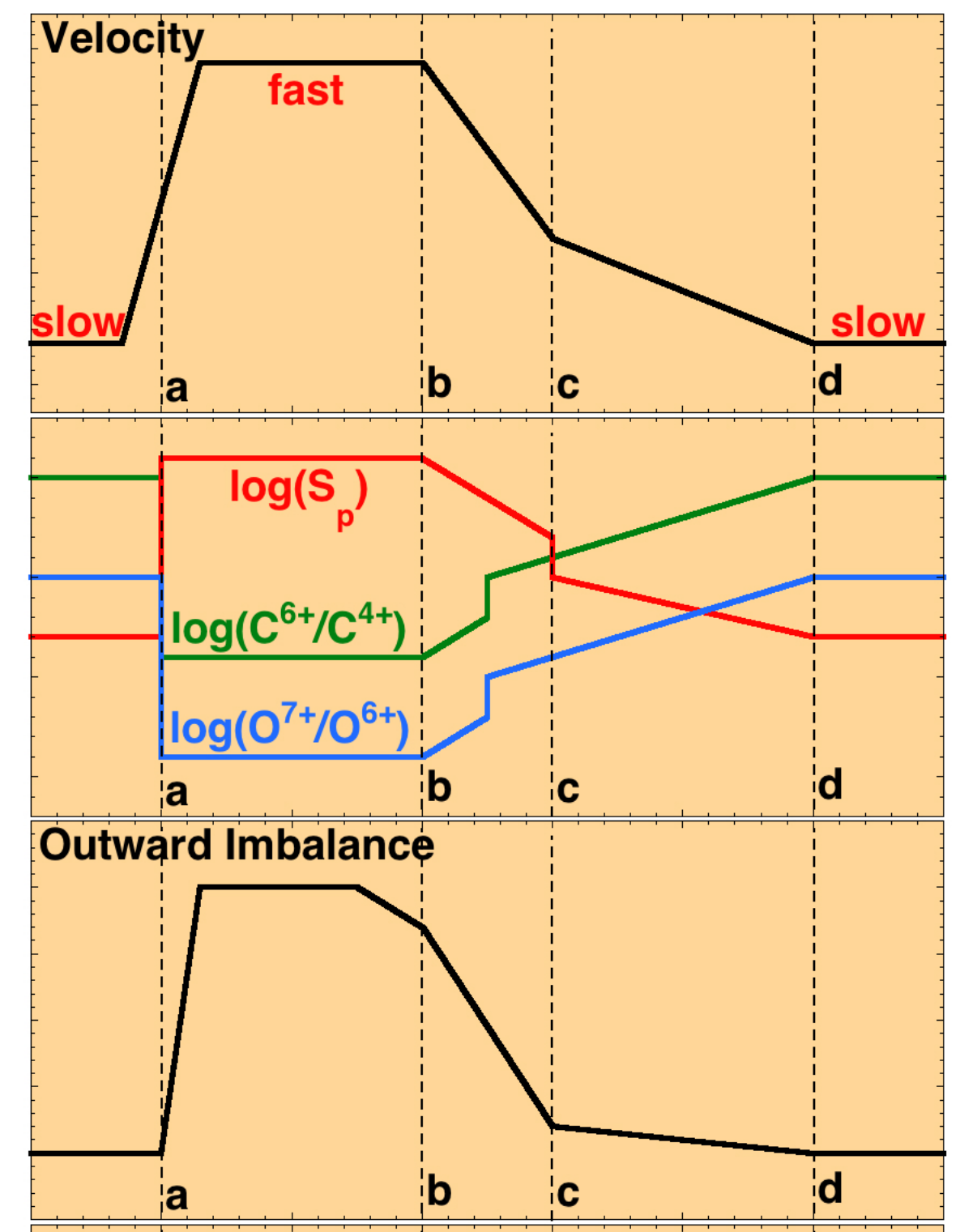

\section{Magnetic-Field Strength}

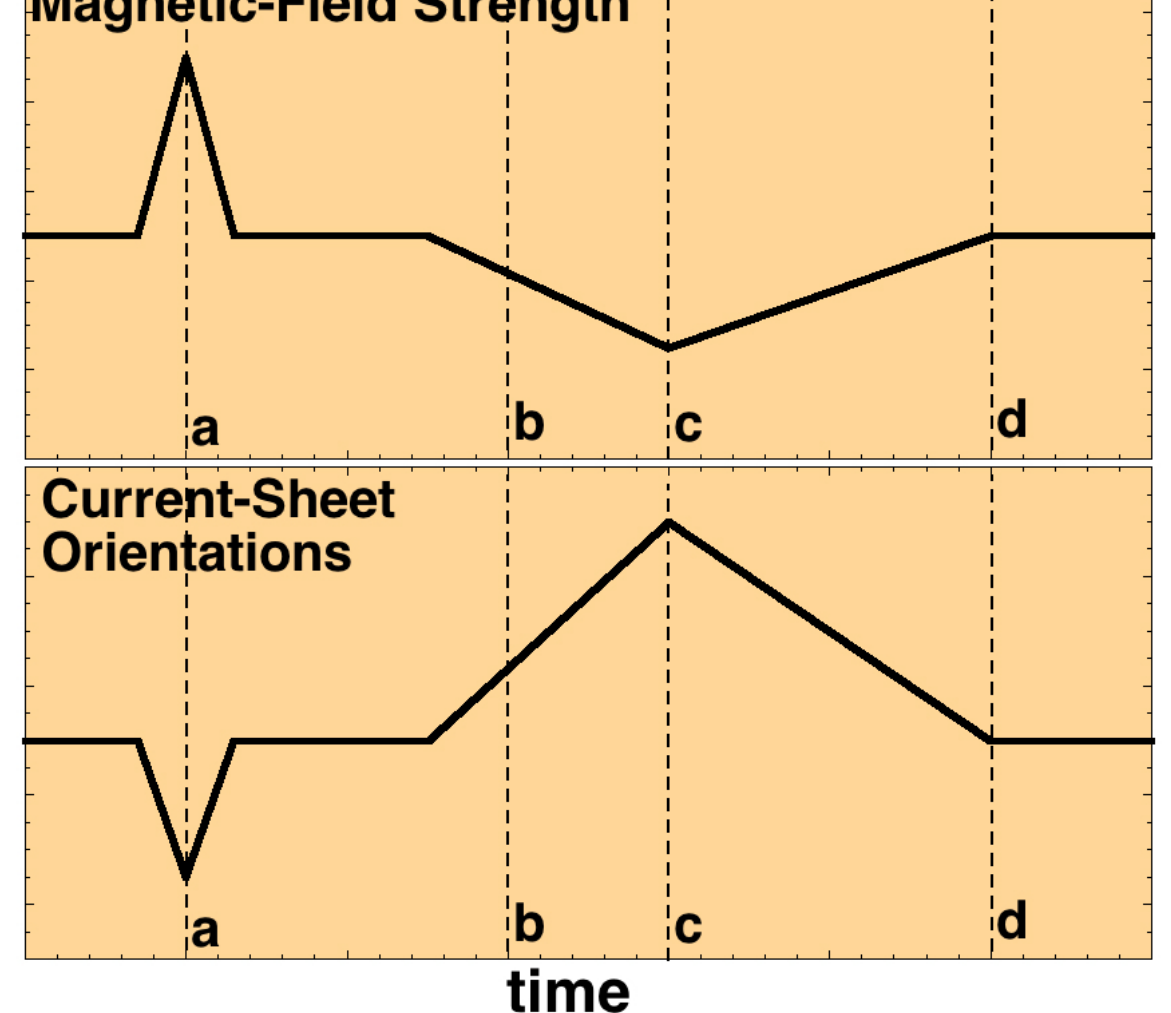




\section{Squashing and Stretching Magnetic Structure}
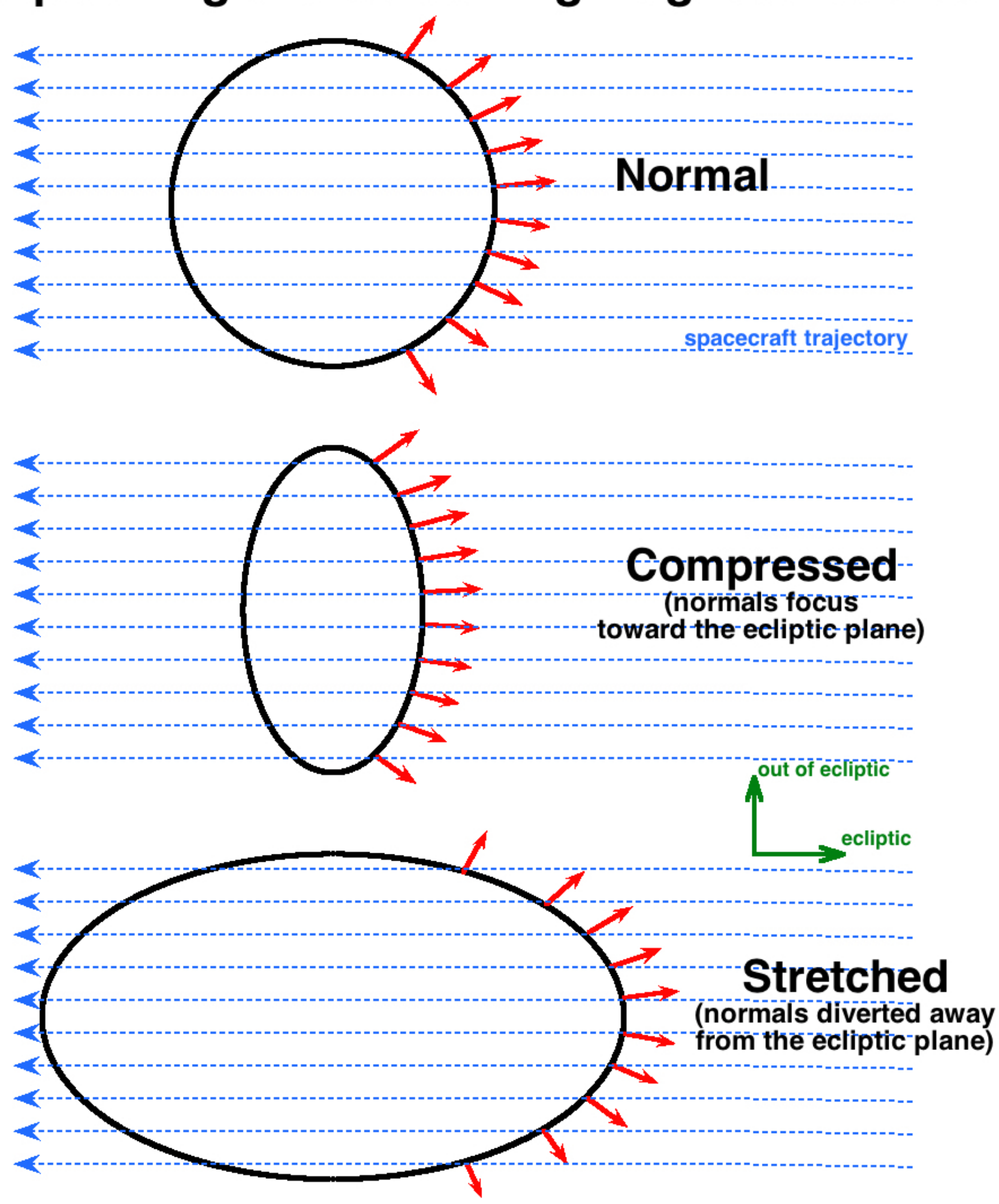

2016JA022863-f24-z-.jpg

This article is protected by copyright. All rights reserved. 


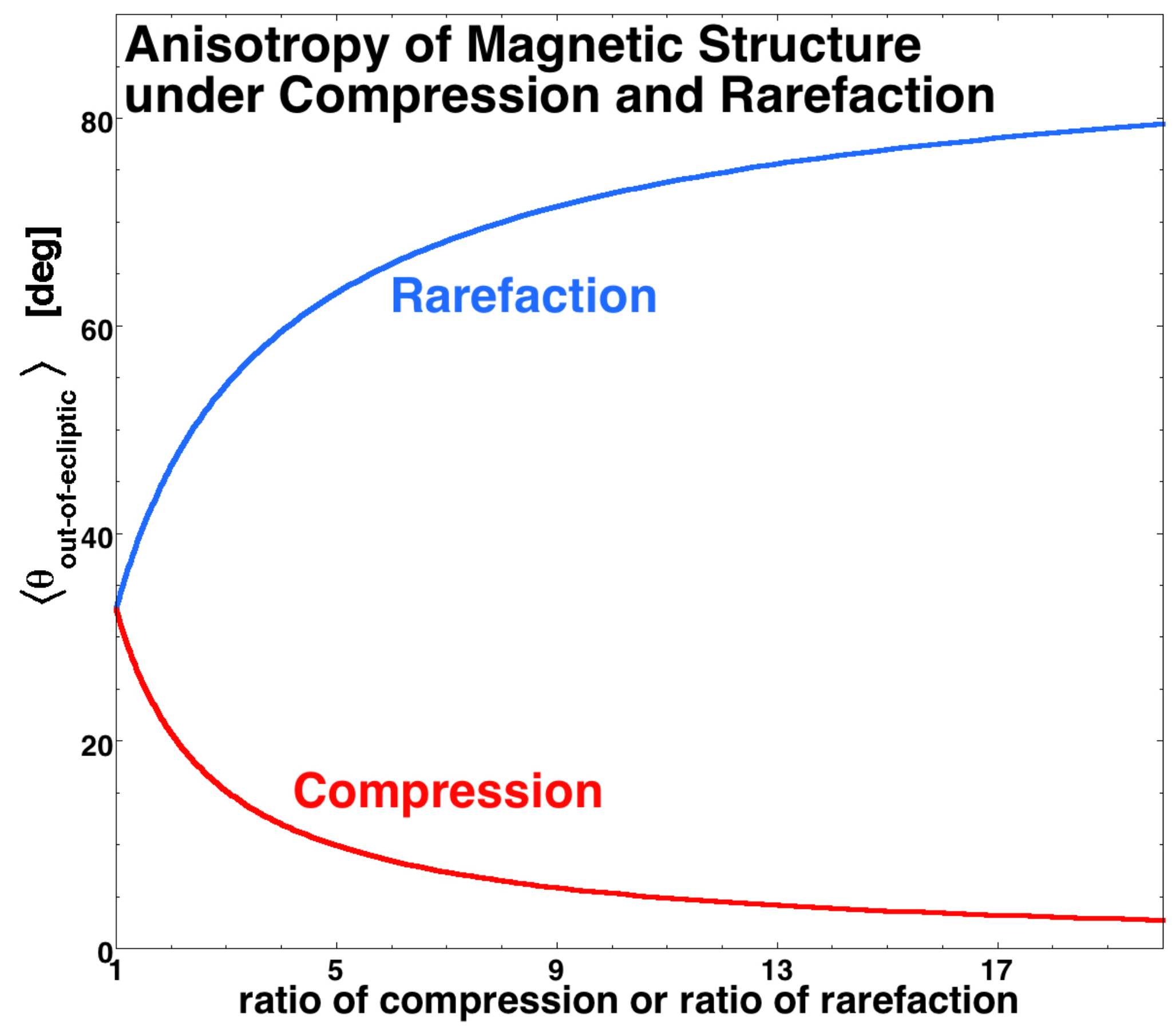

2016JA022863-f25-z-.jpg 